Issued by Sandia National Laboratories, operated for the United States Department of Energy by Sandia Corporation.

NOTICE: This report was prepared as an account of work sponsored by an agency of the United States Government. Neither the United States Government nor any agency thereof, nor any of their employees, nor any of their contractors, subcontractors, or their employees, makes any warranty, express or implied, or assumes any legal liability or responsibility for the accuracy, completeness, or usefulness of any information, apparatus, product, or process disclosed, or represents that its use would not infringe privately owned rights. Reference herein to any specific commercial product, process, or service by trade name, trademark, manufacturer, or otherwise, does not necessarily constitute or imply its endorsement, recommendation, or favoring by the United States Government, any agency thereof, or any of their contractors or subcontractors. The views and opinions expressed herein do not necessarily state or reflect those of the United States Government, any agency thereof, or any of their contractors.

Printed in the United States of America. This report has been reproduced directly from the best available copy.

Available to DOE and DOE contractors from Office of Scientific and Technical Information P.O. Box 62

Oak Ridge, TN 37831

Prices available from (615) 576-8401, FTS 626-8401

Available to the public from National Technical Information Service

U.S. Department of Commerce

5285 Port Royal Rd

Springfield, VA 22161

NTIS price codes

Printed copy: A06

Microfiche copy: A01

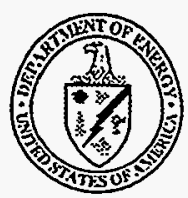




\section{DISCLAIMER}

Portions of this document may be illegible in electronic image products. Images are produced from the best available original document. 
SAND98-2772

Unlimited Release

Printed December 1998

\title{
Enhanced Vapor-Phase Diffusion in Porous Media - LDRD Final Report
}

\author{
Stephen W. Webb \\ Mission Analysis \& Simulation Department \\ Clifford K. Ho \\ Geohydrology Department \\ Sandia National Laboratories \\ P.O. Box 5800 \\ Albuquerque, NM 87185-0977
}

\begin{abstract}
As part of the Laboratory-Directed Research and Development (LDRD) Program at Sandia National Laboratories, an investigation into the existence of enhanced vapor-phase diffusion (EVD) in porous media has been conducted. A thorough literature review was initially performed across multiple disciplines (soil science and engineering), and based on this review, the existence of EVD was found to be questionable. As a result, modeling and experiments were initiated to investigate the existence of EVD.

In this LDRD, the first mechanistic model of EVD was developed, which demonstrated the mechanisms responsible for EVD. The first direct measurements of EVD have also been conducted at multiple scales. Measurements have been made at the pore scale, in a twodimensional network as represented by a fracture aperture, and in a porous medium. Significant enhancement of vapor-phase transport relative to Fickian diffusion was measured in all cases.

The modeling and experimental results provide additional mechanisms for EVD beyond those presented by the generally accepted model of Philip and deVries (1957), which required a thermal gradient for EVD to exist. Modeling and experimental results show significant enhancement under isothermal conditions. Application of EVD to vapor transport in the nearsurface vadose zone show a significant variation between no enhancement, the model of Philip and deVries, and the present results. Based on this information, the model of Philip and deVries may need to be modified, and additional studies are recommended.
\end{abstract}




\section{Table of Contents}

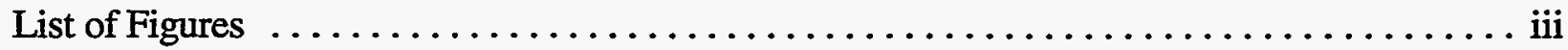

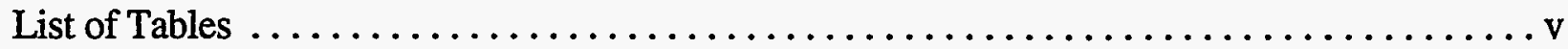

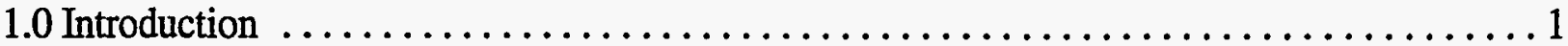

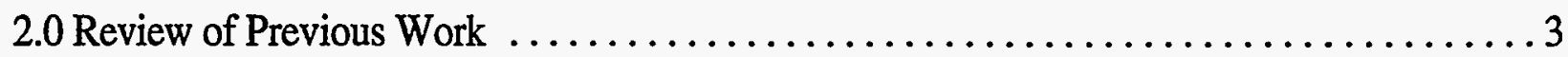

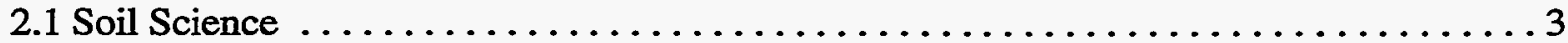

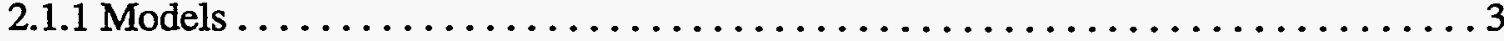

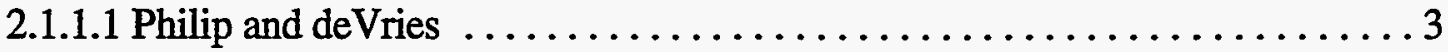

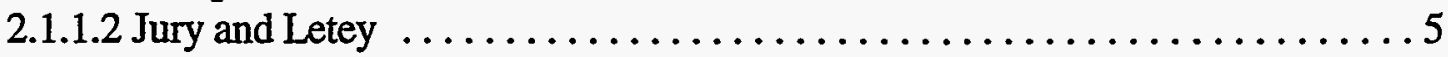

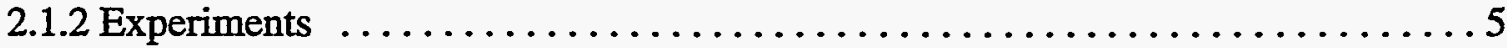

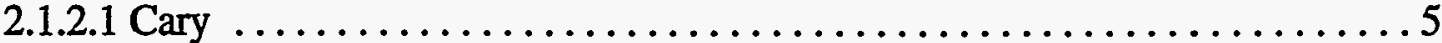

2.1.2.2 Cass et al. ........................................

2.1.3 Data-Model Comparison . . . . . . . . . . . . . . . . . . . . . . . . 6

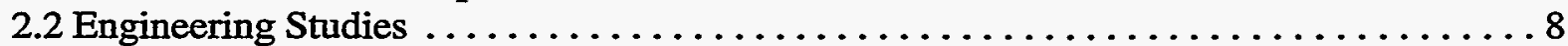

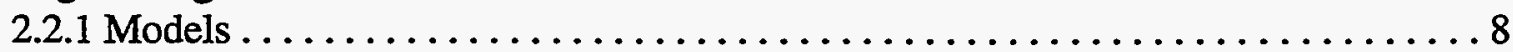

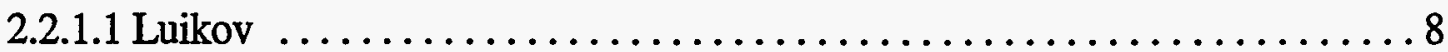

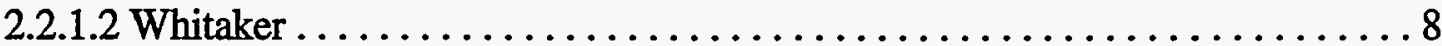

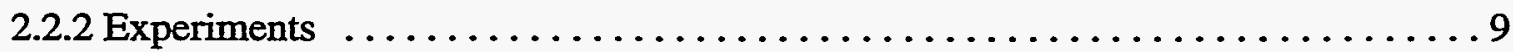

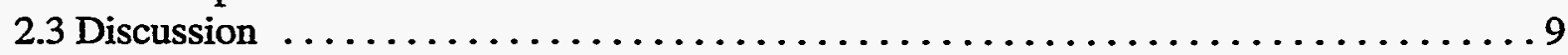

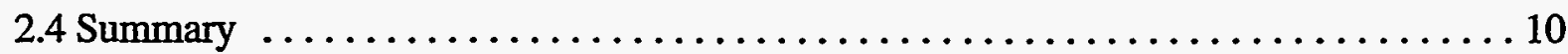

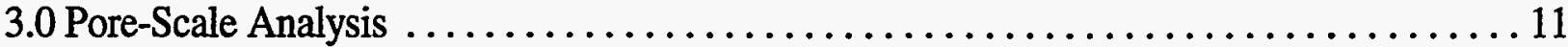

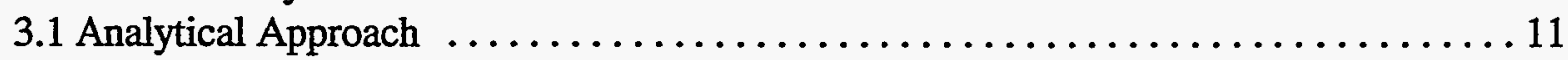

3.1.1 Pore-Scale Analysis - Thermal Gradient ....................... 11

3.1.2 Pore-Scale Analysis - Concentration Gradient $\ldots \ldots \ldots \ldots \ldots \ldots \ldots \ldots \ldots \ldots$

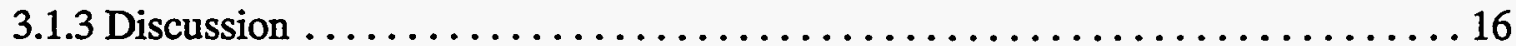

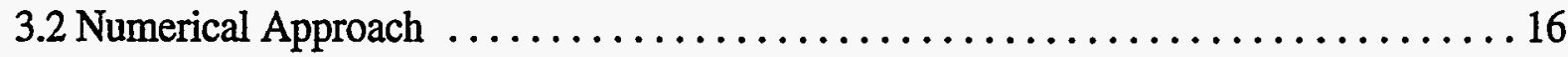

3.2.1 Model Description ...................................... 17

3.2.1.1 Conceptual Model ................................ 17

3.2.1.2 Simplified Representation ............................. 18

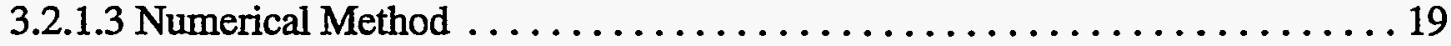

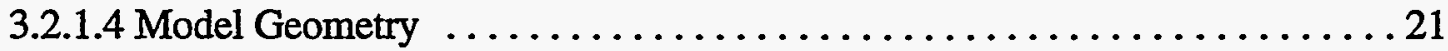

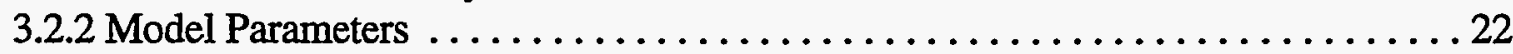

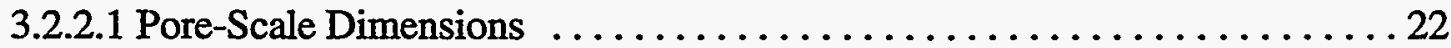

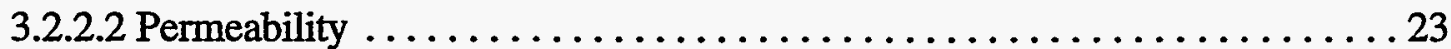

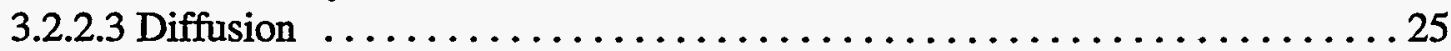

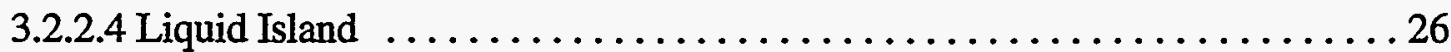

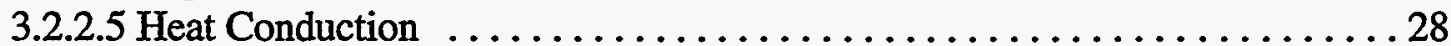

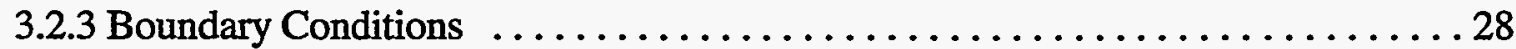

3.2.3.1 Steady-State Concentration Gradient Boundary Conditions .........28

3.2.3.1.1 One-dimensional Linear System . . . . . . . . . . . . . . . 29

3.2.3.1.2 Two-dimensional Single Pore $\ldots \ldots \ldots \ldots \ldots \ldots \ldots \ldots \ldots \ldots \ldots \ldots \ldots \ldots \ldots$

3.2.3.1.3 Two-dimensional Pore Network ........................ 33 


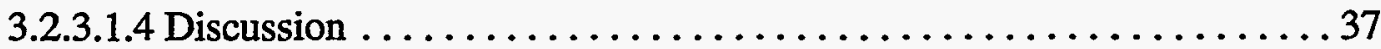

3.2.3.1.5 Conclusions .................................... 39

3.2.3.2 Transient Boundary Conditions - Drying ...................... 39

3.2.3.2.1 With Vapor Pressure Lowering ..................... 40

3.2.3.2.2 Without Vapor Pressure Lowering $\ldots \ldots \ldots \ldots \ldots \ldots \ldots \ldots \ldots . \ldots \ldots$

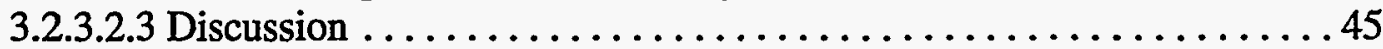

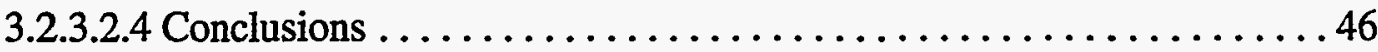

3.2.3.3 Temperature Gradient Boundary Conditions ................. 46

3.2.3.3.1 Results ................................. 47

3.2.3.3.2 Discussion .................................... 51

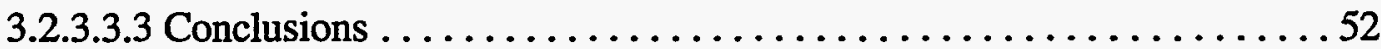

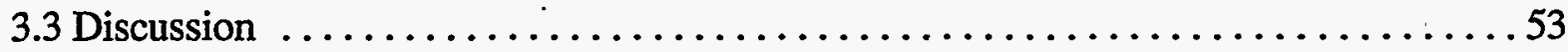

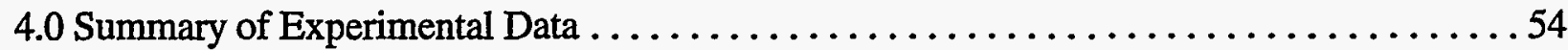

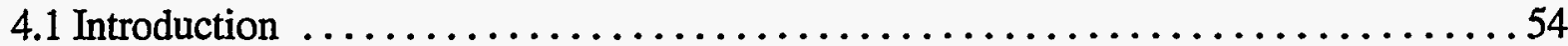

4.2 Pore-Scale Experiments (New Mexico Tech) ....................... 54

4.3 Two-Dimensional Network - Fracture Aperture Experiments (SNL) $\ldots \ldots \ldots \ldots \ldots 58$

4.4 Porous Media Experiments (Washington State University) $\ldots \ldots \ldots \ldots \ldots \ldots \ldots$

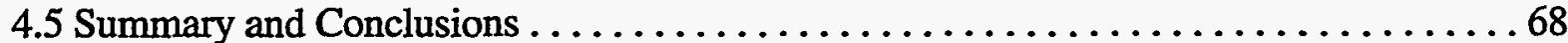

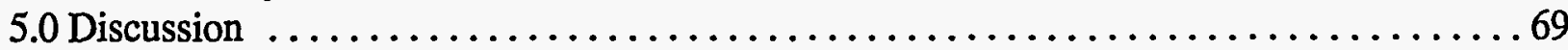

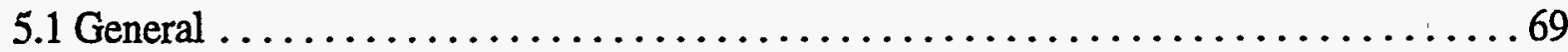

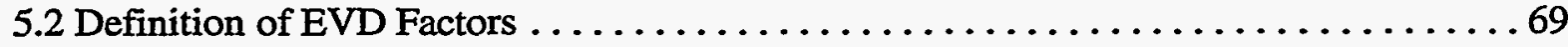

5.3 Comparison of Modeling and Experimental Results $\ldots \ldots \ldots \ldots \ldots \ldots \ldots \ldots \ldots$

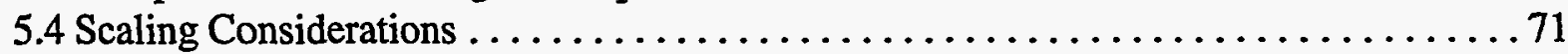

5.5 Vadose Zone Application . . . . . . . . . . . . . . . . . . . . . . . . . . 71

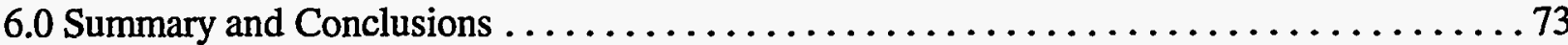

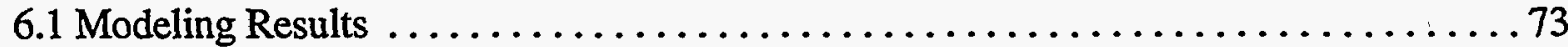

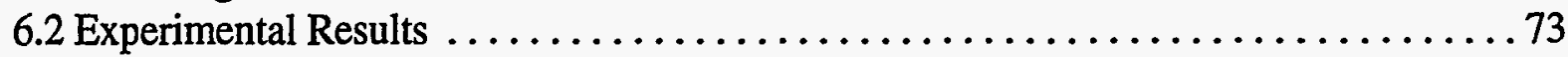

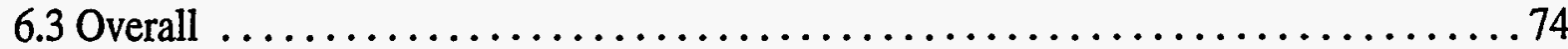

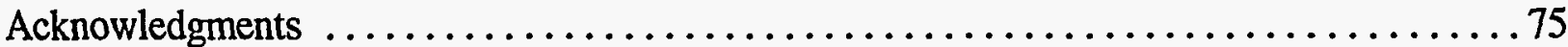

References ............................................... 75

Appendix A - Comparison of Gas Diffusion Models . . . . . . . . . . . . . . . . A

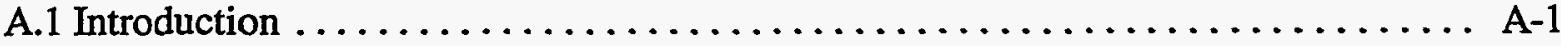

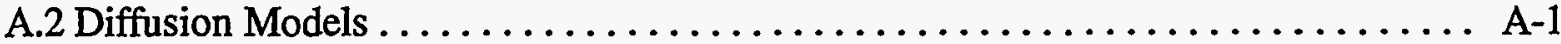

A.2.1 Advective-Dispersive Model $\ldots \ldots \ldots \ldots \ldots \ldots \ldots \ldots \ldots \ldots \ldots \ldots \ldots$ A-2

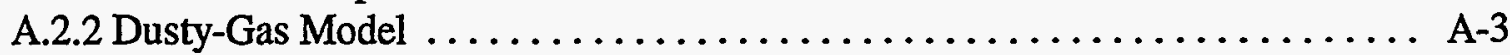

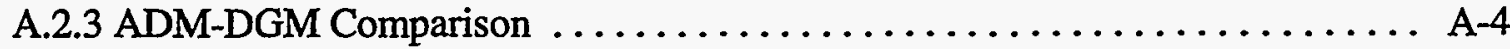

A.2.3.1.Comparison to Fundamental Relationships ................ A-4

A.2.3.2 Evans, Watson, and Truitt Data-Model Comparison ............ A-5

A.2.3.2.1 Zero Pressure Difference $\ldots \ldots \ldots \ldots \ldots \ldots \ldots \ldots \ldots \ldots$ A A-5

A.2.3.2.2 Zero Net Mole Flux $\ldots \ldots \ldots \ldots \ldots \ldots \ldots \ldots \ldots \ldots \ldots \ldots \ldots \ldots \ldots$

A.2.3.2.3 Combined Advection and Diffusion ................ A-7

A.3 Discussion ......................................... A

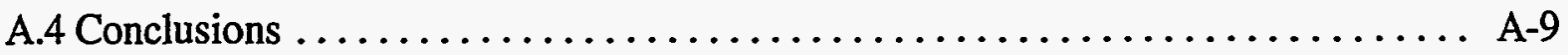

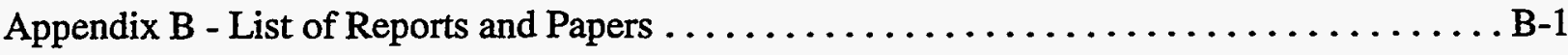




\section{List of Figures}

Figure 2-1 Water Vapor Flux Across Liquid Islands $\ldots \ldots \ldots \ldots \ldots \ldots \ldots \ldots \ldots$

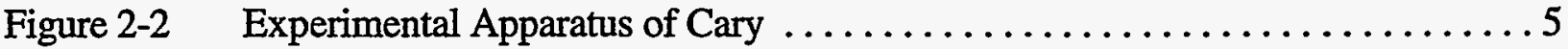

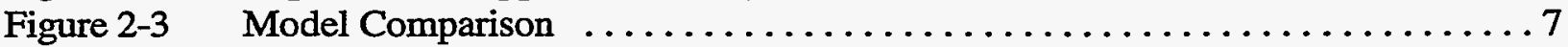

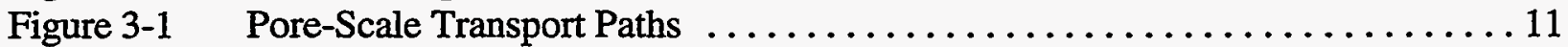

Figure 3-2 Control Surface for Energy Balance $\ldots \ldots \ldots \ldots \ldots \ldots \ldots \ldots \ldots \ldots \ldots \ldots \ldots \ldots \ldots \ldots$

Figure $3-3$ Porous Medium Conceptual Model $\ldots \ldots \ldots \ldots \ldots \ldots \ldots \ldots \ldots \ldots \ldots$

Figure 3-4 Porous Medium Simplified Representation $\ldots \ldots \ldots \ldots \ldots \ldots \ldots \ldots \ldots \ldots \ldots$

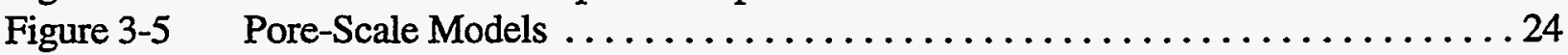

Figure 3-6 Effective Permeabilities for a Parabolic Velocity Profile $\ldots \ldots \ldots \ldots \ldots \ldots . \ldots 25$

Figure 3-7 Definition of Horizontal and Vertical Effective Permeabilities ............ 25

Figure 3-8 Schematic for the Liquid Island Capillary Pressure Function $\ldots \ldots \ldots \ldots \ldots 26$

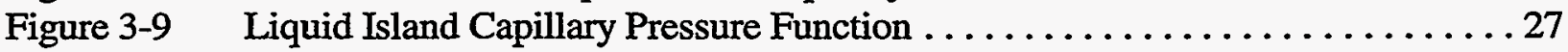

Figure 3-10 Enhancement of Vapor Diffusion for the One-Dimensional Linear Model . . . . 30

Figure 3-11 Enhancement of Vapor Diffusion for the Two-Dimensional Single-Pore

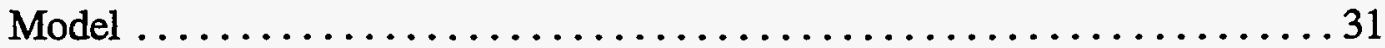

Figure 3-12 Vapor Diffusion Porous Media Factor for the Two-Dimensional Single Pore

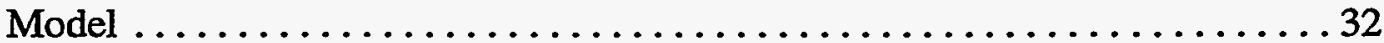

Figure 3-13 Mass Flow Vectors for the Two-Dimensional Pore Network Model for All-Gas

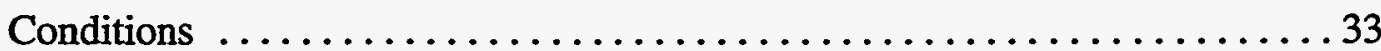

Figure 3-14 Water Vapor Mass Flow Vectors for the Two-Dimensional Pore Network Model

For a Liquid Island Length of $x / R=0.75 \ldots \ldots \ldots \ldots \ldots \ldots \ldots \ldots \ldots \ldots \ldots \ldots$

Figure 3-15 Air Mass Flow Vectors for the Two-Dimensional Pore Network Model For a

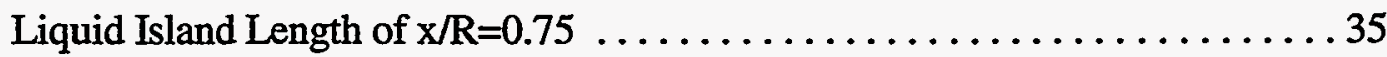

Figure 3-16 Normalized Water Vapor Mass Fraction Profiles .................. 36

Figure 3-17 Enhancement of Vapor Diffusion for the Two-Dimensional Single Pore

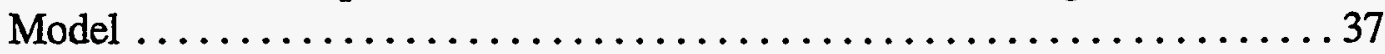

Figure 3-18 Water Vapor and Air Porous Media Factors for the Two-Dimensional Pore

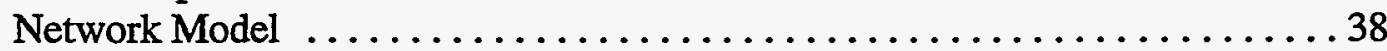

Figure 3-19 Velocity Vectors with VPL for Steady-State Boundary Conditions $\ldots \ldots \ldots \ldots 41$

Figure 3-20 Velocity Vectors with VPL for Transient Boundary Conditions . . . . . . . . . 41

Figure 3-21 Enhancement Factors with Vapor Pressure Lowering $\ldots \ldots \ldots \ldots \ldots \ldots \ldots . \ldots 42$

Figure 3-22 Evaporation Rate with Vapor Pressure Lowering $\ldots \ldots \ldots \ldots \ldots \ldots \ldots . . .42$

Figure 3-23 Velocity Vectors without VPL for Steady-State Boundary Conditions . . . . . . 43

Figure 3-24 Velocity Vectors without VPL for Transient Boundary Conditions . . . . . . . 43

Figure 3-25 Enhancement Factors with and without Vapor Pressure Lowering . . . . . . . . 44

Figure 3-26 Evaporation Rate with and without Vapor Pressure Lowering . . . . . . . . . 44

Figure 3-27 Results for All-Gas Conditions With Concentration and Temperature Gradient

Boundary Conditions . .......................... 47

Figure 3-28 Results for A Liquid Island With Concentration Boundary Conditions . . . . . 48

Figure 3-29 Results for Liquid Island With Concentration Gradient and Temperature Gradient

Boundary Conditions - Aiding Temperature Gradient .............. 49 
Figure 3-30 Results for Liquid Island With Concentration Gradient and Temperature Gradient Boundary Conditions - Opposing Temperature Gradient ............. 49

Figure 3-31 Results for Liquid Island No Concentration Gradient and Opposing Temperature

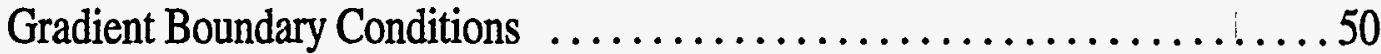

Figure 4-1 Pore-Scale Experimental Apparatus - Single Pore $\ldots \ldots \ldots \ldots \ldots \ldots \ldots \ldots 5$

Figure 4-2 Pore-Scale Experimental Apparatus - Two Pores .................. 56

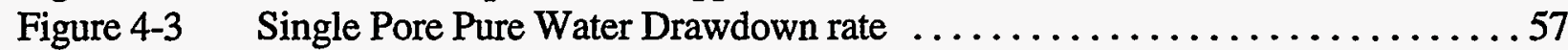

Figure 4-4 Enhancement Factors Vs. Liquid Island Length $\ldots \ldots \ldots \ldots \ldots \ldots \ldots . \ldots \ldots$

Figure 4-5 Light Transmission Experimental System $\ldots \ldots \ldots \ldots \ldots \ldots \ldots \ldots \ldots . \ldots \ldots$

Figure 4-6 Typical liquid island structure at the start of an experiment $\ldots \ldots \ldots \ldots \ldots . .59$

Figure 4-7 Inflow manifold level as a function of time $\ldots \ldots \ldots \ldots \ldots \ldots \ldots \ldots 6 . \ldots \ldots$

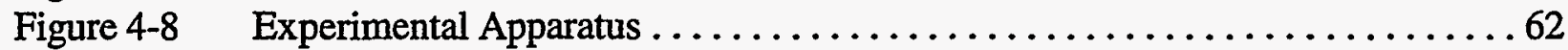

Figure 4-9 Experimental Enhancement Factor, $\eta \ldots \ldots \ldots \ldots \ldots \ldots \ldots \ldots \ldots \ldots \ldots \ldots \ldots \ldots$

Figure 4-10 Experimental Enhancement Factor, $\beta \ldots \ldots \ldots \ldots \ldots \ldots \ldots \ldots \ldots \ldots \ldots \ldots \ldots \ldots$

Figure 4-11 Saturation Profile vs. Time, Transient 1 Experiment $\ldots \ldots \ldots \ldots \ldots \ldots 66$

Figure 4-12 Saturation Profile vs. Time, Transient 2 Experiment $\ldots \ldots \ldots \ldots \ldots \ldots 66$

Figure 4-13 Packed Bed Temperature Profiles $\ldots \ldots \ldots \ldots \ldots \ldots \ldots \ldots \ldots \ldots \ldots . \ldots \ldots$

Figure 4-14 Saturation Profile vs. Time, Drying Experiment $\ldots \ldots \ldots \ldots \ldots \ldots \ldots \ldots$

Figure 5-1 Experimental and Modeling Enhancement Factors, $\eta \ldots \ldots \ldots \ldots \ldots \ldots 70$

Figure A-1 Mole Flux Data-Model Comparison for Zero Pressure Difference

Conditions .................................. A

Figure A-2 Mole Flux Data-Model Comparison for Zero Net Mole Flux Conditions .... A-7

Figure A-3 Pressure Difference Data-Model Comparison for Zero Net Mole Flux Conditions ................................ A-7

Figure A-4 ADM Mole Flux Data-Model Comparison for Combined Advection and Diffusion ................................. A-8

Figure A-5 DGM Mole Flux Data-Model Comparison for Combined Advection and

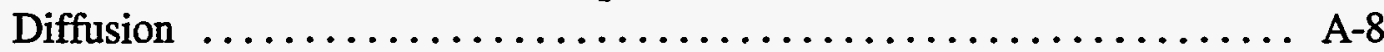




\section{List of Tables}

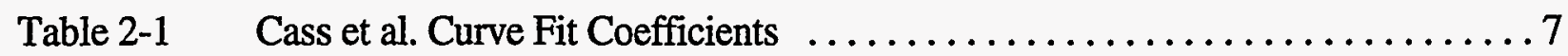

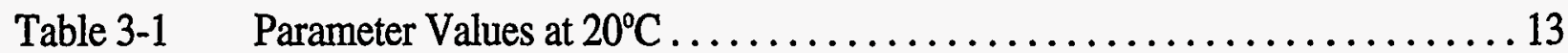

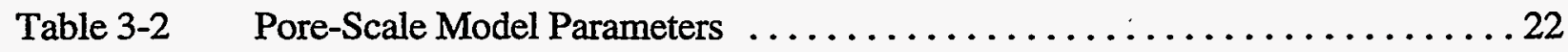

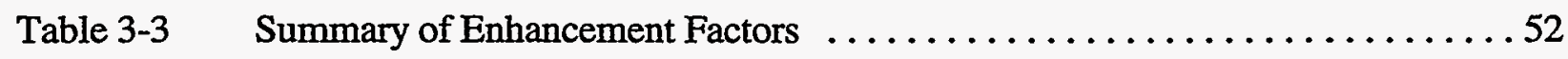

- vi- 


\section{Nomenclature}

$\begin{array}{ll}\text { A } & \text { constant in equation (2-2); area } \\ \mathrm{b} & \text { Klinkenberg factor } \\ \mathrm{B} & \text { constant in equation (2-2) } \\ \mathrm{C} & \text { constant in equation (2-2); concentration; specific heat } \\ \mathrm{D} & \text { constant in equation (2-2) } \\ \mathrm{D}^{*} & \text { defined by equation (3-20) } \\ \mathrm{D}_{\mathrm{i}}^{\mathrm{K}} & \text { Knudsen diffusion coefficient for gas i } \\ \mathrm{D}_{\mathrm{ij}} & \text { effective binary diffusion coefficient for gas components } \mathrm{i} \text { and } \mathrm{j} \\ \mathrm{D}_{12}{ }^{0} & \text { binary diffusion coefficient in free space at } 1 \text { bar and } 0^{\circ} \mathrm{C} \text {. } \\ \mathrm{E} & \text { constant in equation }(2-2) \\ \mathrm{EVD} & \text { enhanced vapor diffusion } \\ \mathrm{f} & \text { clay content } \\ \mathrm{f}_{\mathrm{VPL}} & \text { vapor pressure lowering factor } \\ \mathrm{F} & \text { mass flux } \\ \mathrm{g}_{\mathrm{c}} & \text { gravity } \\ \mathrm{h} & \text { enthalpy; coordinate measured from the center of the channel } \\ \mathrm{h}_{\mathrm{fg}} & \text { latent heat } \\ \mathrm{J} & \text { mole flux } \\ \mathrm{J}_{\mathrm{iD}} & \text { ordinary diffusion mole flux } \\ \mathrm{J}_{\mathrm{iK}} & \text { Knudsen diffusion mole flux } \\ \mathrm{k} & \text { permeability } \\ \mathrm{k}_{\mathrm{B}} & \text { Boltzmann's constant } \\ \mathrm{K} & \text { thermal conductivity } \\ \mathrm{L} & \text { linear distance } \\ \mathrm{M} & \text { mass accumulation term; molecular weight } \\ \mathrm{m}_{\mathrm{v}} & \text { vapor mass flow rate } \\ \mathbf{n} & \text { normal vector } \\ \Delta \mathrm{P} & \text { pressure difference } \\ \mathrm{P} & \text { pressure } \\ \mathrm{q} & \text { source } \\ \mathrm{r} & \text { pore radius } \\ \Delta \mathrm{R}_{\mathrm{c}} & \text { change in radius of curvature } \\ \mathrm{R} & \text { universal gas constant; particle radius } \\ \mathrm{S} & \text { saturation } \\ \mathrm{SNL} & \text { Sandia National Laboratories } \\ \mathrm{t} & \text { time } \\ \mathrm{T} & \text { temperature } \\ \Delta \mathrm{T} & \text { temperature difference } \\ \mathrm{u} & \text { internal energy } \\ \mathrm{v} & \text { mean molecular speed } \\ \mathrm{V} & \text { volume } \\ \mathrm{X} & \text { distance; mole fraction } \\ & \end{array}$




\section{Greek}

$\beta$

$\rho$

$\kappa, \kappa^{*}$

$\sigma$

$\tau$

$\varphi$

$\omega$

$\Gamma$

$\mu$

$\eta$

$\theta$

$\lambda$

porous media factor; phase indicator

density

component

surface tension

tortuosity coefficient

porosity

mass fraction

surface

viscosity

mechanistic enhancement factor

moisture content

thermal conductivity

Subscripts

c

EVD

Fick

g

i

1

liquid island liquid island

p, Pore

$\mathbf{r}$

R

sat

$t$

v

w

0

1,2

$\kappa, \kappa^{*}$

capillary

enhanced vapor diffusion

Fickian

gas

gas $i$; species or component $i$

liquid

pore

relative

rock

saturated

throat

vapor

water

reference value

species or component number, coordinate

component

Superscripts

ADM

Advective-Dispersive Model

DGM

Dusty-Gas Model 


\subsection{INTRODUCTION}

Gas diffusion in porous media is generally significantly smaller than in free space due to the presence of the porous medium. The flow area for gas-phase diffusion is reduced by the presence of the solid particles, by the presence of any liquid, and by the fact that the flow path for diffusion in a porous medium is more tortuous than in free space. Using Fick's law, gas diffusion in a porous media may be expressed as

$$
F_{i}=-\tau \varphi S_{g} D_{12} \rho_{g} \nabla \omega_{i}=-\beta D_{12} \rho_{g} \nabla \omega_{i}
$$

where $D_{12}$ is the free-space diffusion coefficient at the pressure and temperature of interest. The product of the tortuosity coefficient, $\tau$, the porosity, $\varphi$, and the gas saturation, $S_{g}$, is often referred to as the porous media factor, $\beta$. The porous media factor, $\beta$, is always less than 1 , and gas diffusion in a porous medium is usually much lower than in free space.

In contrast, it has been postulated that diffusion of a condensible vapor in the presence of its liquid may be considerably enhanced compared to gas diffusion rates and may approach or exceed free-space values. (In the present discussion, gas refers to a non-condensible inert gas under the conditions of interest, e.g. air. Vapor refers to the gas phase of a condensible substance which may have a liquid phase present, e.g. water vapor). The mechanisms for such an enhancement are postulated to occur at the pore scale and include local condensation and evaporation at isolated liquid "islands" within the porous medium, and an increased temperature gradient in the gas phase compared to the average temperature gradient in the equivalent porous medium. Enhanced vapor diffusion (EVD) was first considered by Philip and deVries (1957) for soils. Jury and Letey (1979) estimated that the value of $\beta$ is of the order 1 resulting in considerable enhancement of vapor diffusion compared to gas diffusion. When $\beta$ equals 1 , diffusion is not affected by the porous medium at all and is equal to the value for free-space diffusion. Enhanced vapor diffusion is commonly assumed in soil science but has seen limited use in engineering applications.

Ho and Webb $(1996,1998)$ reviewed previous EVD studies and came to the conclusion that, while enhanced vapor diffusion may exist, there was no direct evidence to support it. Only indirect evidence existed, where the enhancement has been inferred from other measurements such as moisture content or heat flux. In order to answer the question, Ho and Webb recommended additional modeling and experiments at multiple length scales. As part of the present investigation, a number of modeling and experimental studies have been conducted which are summarized in this LDRD final report as follows: 
Section 1.0 - Introduction (current section)

Section 2.0 - Review of Previous Work

Section 3.0 - Pore-Scale Modeling - As part of this LDRD, the first mechanistic model for EVD was developed. This model is described along with results for various boundary conditions.

Section 4.0 - Experimental Data - The first direct measurements of EVD were performed as part of this investigation. These measurements were taken at three different length scales - pore-scale, a two-dimensional network, and a porous media. These data are summarized in this section.

Section 5.0 - Discussion - Section 5.0 discusses the accomplishments of this LDRD and some of the important implications of this research.

Section 6.0 - Summary and Conclusions

Appendix A - Comparison of Gas Diffusion Models - Additional work was done on modeling gas diffusion in porous media to provide a sound basis for the analysis of vapor diffusion.

Appendix B - List of Reports and Papers - This appendix summarizes the reports and papers produced as part of this LDRD.

The material presented in this report is of a summary nature in order to prevent duplication of information; details are provided in referenced technical reports and papers which are listed in Appendix B. The set of reports and papers are available from the lead author. 


\subsection{REVIEW OF PREVIOUS WORK}

As discussed by Ho and Webb $(1996,1998)$, vapor diffusion in porous media has been studied in many diverse disciplines ranging from soil science to engineering. The applications that motivated these studies are also diverse, ranging from soil heating problems in agriculture to drying of textiles and foodstuffs in engineering and manufacturing. Unfortunately, the diversity of the applications and disciplines has led to a potpourri of analyses and solution techniques associated with heat and moisture transport in porous media.

The most important modeling and experimental data for EVD are from the soil science area. The "mechanistic" model of Philip and deVries (1957) stands out, in part because it is the first model to discuss the mechanisms of EVD, and in part because the model is widely used even today. Other important modeling contributions include Jury and Letey (1979), who! quantified the value of enhancement which has been widely cited. Much of the experimental work in soil science was conducted by Cary and coworkers over a number of years. In addition, Cass et al. (1984) obtained data which indicated the variation of EVD with saturation. These contributions are reviewed in more detail below. Ho and Webb $(1996,1998)$ summarize additional contributions to the field.

Note that there are two different enhancement factors. The two types are a mechanistic enhancement factor, $\eta$, which is the enhancement of the vapor diffusion rate compared to the gas diffusion rate, and the porous media factor, $\beta$, which is relative to diffusion in free space. The two factors are simply related by the gas diffusion values of tortuosity, porosity, and gas saturation, or

$$
\beta=\tau \varphi S_{g} \eta .
$$

\subsection{Soil Science}

\subsubsection{Models}

\subsubsection{Philip and deVries}

The first widely accepted analysis for EVD came from Philip and deVries (1957), who introduced a theory for heat and moisture movement in porous materials. They assumed that the moisture is transported in both the gas and liquid phases under moisture and temperature gradients in the soil.

Philip and deVries postulated a number of mechanisms that enhance vapor transport compared to gas diffusion. The first mechanism includes pore-level phase change effects in liquid islands between solid particles. The postulated transport of water across liquid islands 


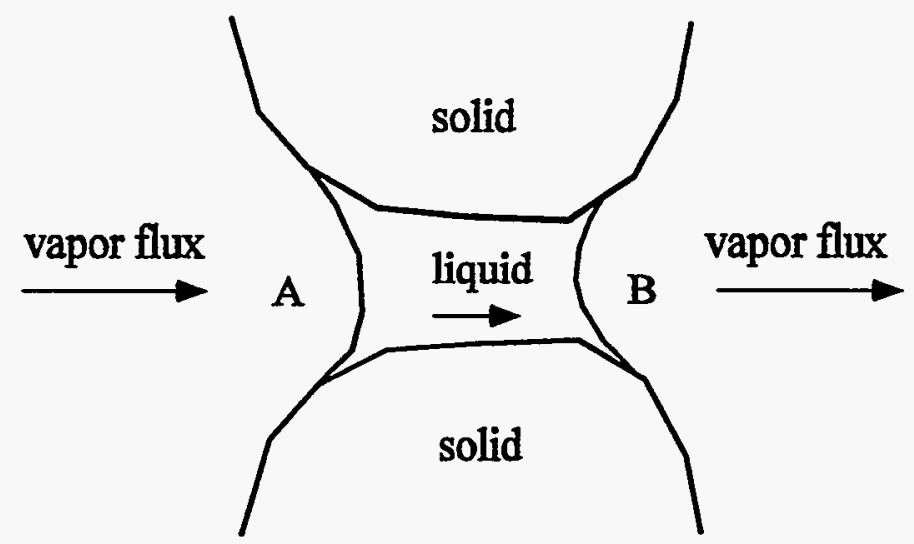

Figure 2-1

Water Vapor Flux Across Liquid Islands

is due to condensation of water vapor on the "hot" side of the liquid island and subsequent evaporation on the "cool" side. The curvature of the liquid decreases at A and increases at B, causing capillary driven transport of the liquid from A to B. The resulting liquid flow through the island equals the rate of evaporation and condensation, and the total flux adjusts itself to equal the vapor flux in the air-filled pores. This mechanism tends to decrease the tortuosity and increase the effective flow area for the vapor phase compared to a gas component and is discussed in more detail in section 3.0 of this report. The second mechanism considers local temperature gradients in the air-filled pores, which may be significantly higher than the average temperature gradient as a result of different thermal conductivities. Both of these postulated mechanisms act to increase the value of the thermal vapor diffusivity. Note that Philip and deVries assume that enhancement occurs in the presence of a temperature gradient. If there is no temperature gradient, there is no EVD.

The Philip and deVries (1957) model has been used by numerous investigators even today including Milly (1982, 1984a,b, 1996), Shurbaji and Phillips (1995), Braud et al. (1995) and Nassar and Horton (1997). (Note that Philip and deVries, 1957, ignored the dependence of $h$ on temperature as corrected by Nakano and Miyazaki, 1979).

The application of the enhancement factor exclusively to the thermal gradient term is due to the perceived dominance of the temperature gradient on the proposed mechanisms for enhanced vapor diffusion. Pore-scale modeling and some ongoing experiments, which will be discussed later, indicate some additional mechanisms for EVD; enhanced vapor diffusion is seen even if no temperature gradient is imposed. Milly (1997, personal communication) also speculated that the enhancement factor may apply to the other terms, although he only applied it to the thermal gradient in his analyses (Milly, 1982, 1984a,b, 1996). 


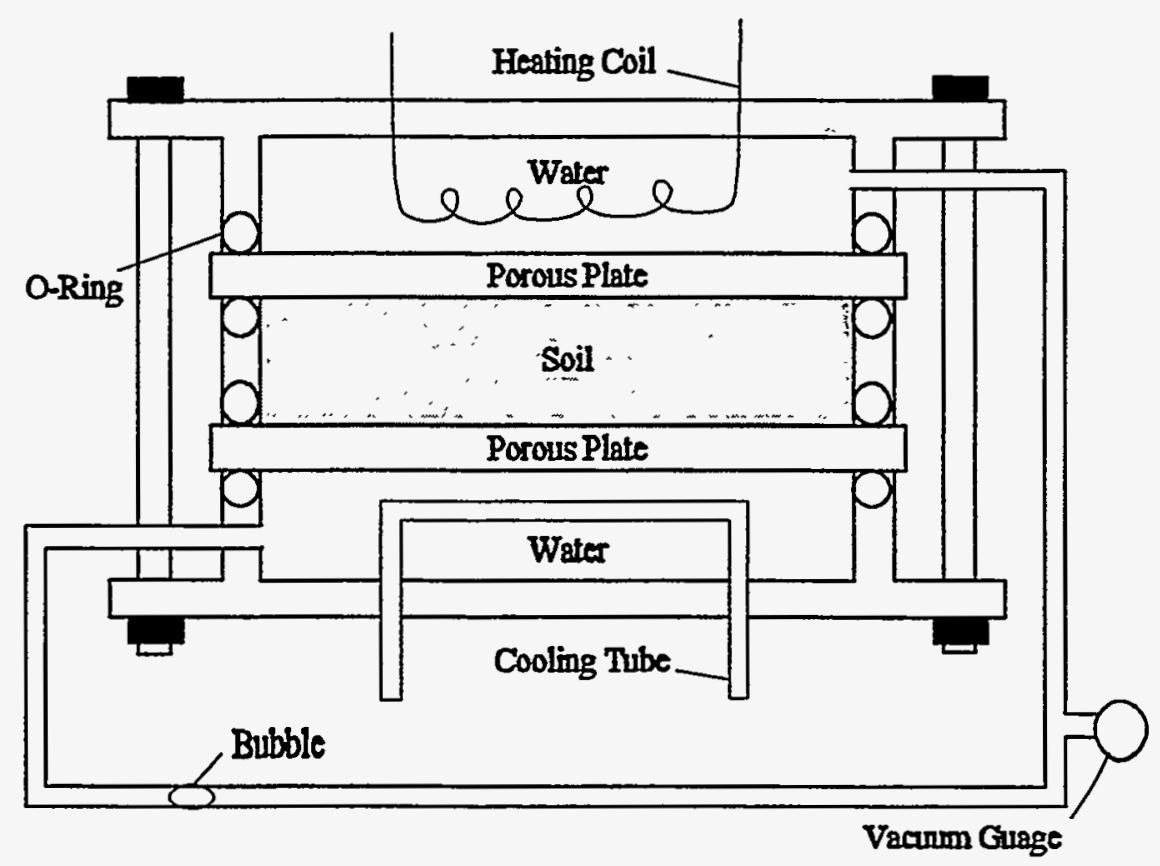

Figure 2-2

Experimental Apparatus of Cary

\subsubsection{Jury and Letey}

One of the most influential papers in promoting the use of the enhanced vapor diffusion coefficient is from Jury and Letey (1979). In that paper, they interpreted the results of several experiments (many from Cary) and determined the enhancement factor for vapor diffusion. They concluded that the enhancement factor $\beta\left(=\tau \varphi S_{g}\right)$ ranged between 1.0 and 3.5 for a number of experiments. They also claimed that a fixed value of $\beta$ could be used $(\beta=1.83)$ in most circumstances that would predict vapor transport within a factor of 2 .

\subsubsection{Experiments}

\subsubsection{Cary}

From 1962 to 1979 , Cary published a number of papers that investigated thermally driven moisture transport in porous media. He derived the expressions for vapor and heat flow based on the thermodynamic theory of irreversible processes (Cary and Taylor, 1962a\&b). Cary backcalculated the necessary coefficients for his models from his numerous experiments (Cary and Taylor, 1962a\&b, Cary, 1963, 1964, 1965, 1979). His most important contribution is the large number of experiments he conducted.

Figure 2-2 shows a sketch of the experimental apparatus that produced much of the experimental data that he and others have used to verify formulations of heat and moisture transport and to demonstrate the existence of enhanced vapor diffusion. In these experiments, a 
soil sample is held between two porous ceramic plates. Above and below the ceramic plates are water reservoirs that are heated and cooled, respectively, to yield constant temperatures. The water reservoirs are connected by an external tube that contains an air bubble. A pressure difference can be applied across the two reservoirs. The thermal gradient established by the two water baths induced a flow of water (liquid and vapor) through the soil sample and through the external flow tube. The total water flux was measured by the movement of the air bubble through the calibrated flow tube that connected the two water reservoirs. Temperatures were also recorded at several locations within the soil sample. Slight variations to this apparatus were made by Cary between 1962 and 1979, but the general principle remained the same. Vapor diffusion rates were calculated from the total water movement and the heat flux data. To estimate the thermal flow component, pressure-driven flow was calculated and subtracted from the total water movement. The measured temperature dependence of the heat flux, along with the thermal component of water (liquid + vapor) was used to infer the vapor diffusion rate.

\subsubsection{Cass et al.}

Most of the inferred values of enhanced vapor diffusion, such as by Cary (1965) who deduced vapor movement from total water movement, are at discrete values for different average temperatures and moisture contents. No continuous variation of a parameter, such as the moisture content, was performed. The data from Cass et al. (1984) are significantly different. Their data, which inferred vapor diffusion rates from heat flux/thermal conductivity information, give a continuous variation in the vapor diffusion rate, and the enhancement, as a function of moisture content at different temperatures. Their empirical fit, which is discussed later, is also important because it considers the behavior of the enhancement factor at various moisture content limits.

\subsubsection{Data-Model Comparison}

The models of Philip and deVries (1957) and Jury and Letey (1979) were described above. These models are compared to the curve fits of data presented by Cass et al. (1984) and Campbell (1985) in this section. Also presented is a model by Cary (1979). Note that all the models apply the enhancement to the vapor flux due to a thermal gradient only.

Cass et al. (1984) presented curve fits to their data in the form

$$
\eta=A+B S_{l}-(A-D) \exp \left[-\left(C S_{l}\right)^{E}\right]
$$

and, $\beta$ can be calculated from

$$
\beta=\tau \varphi S_{g} \eta
$$


Table 2-1. Cass et al. (1984) Curve Fit Coefficients

\begin{tabular}{|l|c|c|c|c|c|}
\hline & A & B & C & D & E \\
\hline Lysimeter Sand, $3.5^{\circ} \mathrm{C}$ & 13.5 & 3 & 10 & 1.3 & 3 \\
\hline Lysimeter Sand, $22.5^{\circ} \mathrm{C}$ & 9.5 & 2 & 8 & 0.5 & 3 \\
\hline Lysimeter Sand, $32.5^{\circ} \mathrm{C}$ & 8.0 & 3 & 10 & 1.0 & 3 \\
\hline Portneuf Silt Loam, $32.5^{\circ} \mathrm{C}$ & .9 .5 & 3 & 3.5 & 1.0 & 4 \\
\hline
\end{tabular}

Their curve fit considers the limits at low liquid saturations such that the value of $\eta$ should approach 1 at zero liquid saturation, and $\eta$ should approach 0 for liquid-saturated conditions. The experimental data are consistent with these expectations. The experimental values of $A, B, C, D$, and $E$ are given in Table 2-1.

Campbell (1985) proposed a fit which is presumably based on the Cass et al. (1984) data:

$$
\eta=9.5+6 \theta-8.5 \exp \left[-\left[\left(1+2.6 f^{-1 / 2}\right) \theta\right]^{4}\right]
$$

where $\theta$ is the moisture content $\left(\varphi S_{1}\right)$, and $f$ is the clay fraction content.

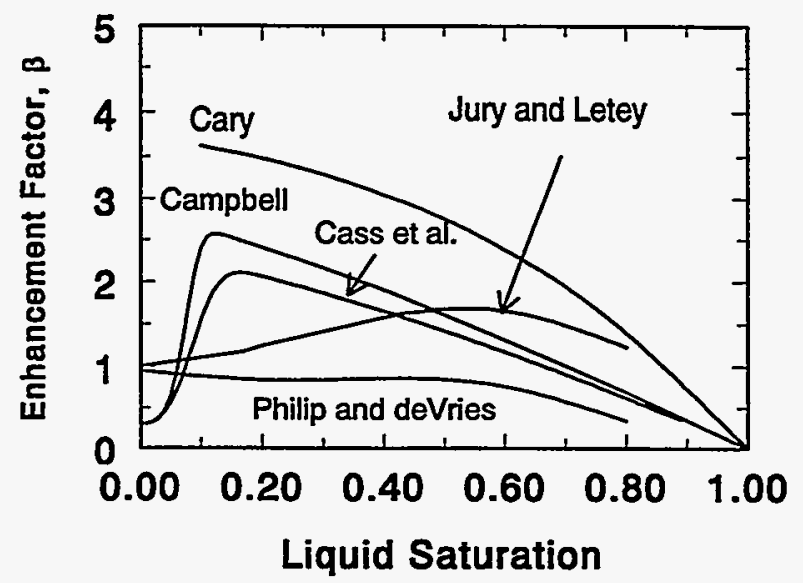

Figure 2-3

Model Comparison

The values of $\beta$ from the various models for sand are compared in Figure 2-3. Cass et al. (1984) did a similar comparison for their correlation and the models of Cary (1979) and Jury and Letey (1979). The prediction of Cary (1979) is taken from the Cass et al. (1984) plots. As discussed above, Cass et al. (1984) argued that $\beta$ should approach $\tau \varphi$ at zero liquid saturation, and should approach zero at full liquid saturation. As can be seen, only the correlation of Cass et 
al. (1984) and the fit of Campbell (1985) adhere to this dramatic variation in the porous media factor at low liquid saturations, where enhanced vapor diffusion is most important.

The long history and acceptance of EVD in soil science has prompted the use of this enhancement factor in recent works ranging from geomorphic investigations of the planet Mars (Clifford, 1991) to performance assessments of high-level radioactive waste disposal (Pruess and Tsang, 1993). However, no direct measurements of enhanced vapor diffusion have been made. For example, Jury and Letey (1979) referenced the column experiments of Weeks et al. (1968), who performed heat and moisture transport experiments in horizontal columns of soil. Only temperatures and suctions were measured at several locations in the experiment, which had its ends held at constant hot and cold temperatures. To calculate the value of the enhancement factor, $\beta$, they assumed that only vapor-phase flow existed. Effects of liquid transport were not considered, even though they acknowledged its existence.

\subsection{Engineering Studies}

\subsubsection{Models}

Many engineering studies investigating heat and moisture transport in porous materials can be found in the drying literature. Drying of various materials ranging from textiles to foodstuffs encompasses many of the processes that are also investigated by soil science researchers. However, the engineering discipline has not embraced the area of EVD as fully as the soil science discipline. This section reviews the significant contributions made in the area of heat and moisture transport in porous media as applied to drying theory in the engineering discipline.

\subsubsection{Luikov}

Russian researchers have credited Luikov with discovering the phenomenon of moisture thermal diffusion and establishing the importance of temperature gradients on causing moisture transfer in porous materials (Lebedev, 1961; Mikhailov, 1973). In his theory, Luikov employs the theory of irreversible thermodynamics to derive expressions for liquid and vapor flow (Luikov, 1975). The resulting flux expressions are similar to the equations derived by Philip and deVries (1957). However, Luikov does not postulate the possibility of enhanced vapor diffusion by any of the mechanisms proposed by Philip and deVries (1957).

\subsubsection{Whitaker}

Whitaker developed a comprehensive set of equations governing the simultaneous transport of mass, momentum, and heat in a porous medium (Whitaker, 1977). He derives these equations using volume-averaged quantities in the balance laws for mass, momentum, and energy. By integrating quantities over a representative elementary volume, the local equations of transport can be transformed into macroscopic equations for combined mass, momentum, and 
energy transport in a porous medium. No mention is made of enhanced vapor diffusion in his work. We have discussed our pore-scale modeling results with Professor Whitaker. Future reviews on this subject by Whitaker may include references to some of the work presented in this report (Whitaker, personal communication, 1997).

\subsubsection{Experiments}

Numerous experiments have been described in the engineering literature pertaining to the movement of moisture in porous media. A large number of these pertain to the drying of porous materials such as soil or foodstuffs. A famous paper by Ceaglske and Hougen (1937) investigated the one-dimensional drying of wet sand.

In most of the experiments, the porous media starts out fully saturated and undergoes a drying transient. Even though detailed mechanistic models are sometimes used to interpret the data, enhanced vapor diffusion is not important during most of the drying process because it occurs at high liquid saturations. EVD will only be significant at lower liquid saturations where vapor transport is significant.

\subsection{Discussion}

The existing models of EVD presented in past studies are qualitative; no mechanistic model of EVD has yet been developed. In addition, the data for EVD are inferred from other measurements, such as total water movement or thermal conductivity/heat flux. Therefore, based on the review of the current literature by Ho and Webb $(1996,1998)$, it was recommended that a mechanistic model for EVD be developed, and that direct measurements of EVD be performed.

In order to try to decide the appropriate experiments to conduct to try to measure EVD in a porous media, Ho and Webb $(1996,1998)$ modeled the two types of experiments typically performed in the soil science and engineering literature. In the first experiment, a capped column of sand similar to the experiments of Cary (1965) was used to compare predicted and measured moisture content profiles during an imposed temperature gradient. The second experiment compares predicted and measured saturation and temperature profiles for external convective drying of an open-ended column of glass beads, similar to the experiment performed by Ceaglske and Hougen (1937). These two experiments were chosen because of the availability of the material and system parameters that were reported. The sensitivity of experimentally measured quantities on the effects of enhanced vapor diffusion were examined in these different systems.

The closed-end systems similar to Cary were insensitive to the existence of enhanced vapor diffusion. Measured values of the moisture content, which were frequently used to determine total water movement and to infer vapor movement showed very little change when EVD was included in the model. 
In contrast, the open-ended, transient drying experiment were very sensitive to the diffusion coefficients. However, the high initial saturation of this experiment and the heating configuration did not allow for rigorous assessment of the enhanced vapor diffusion coefficient. Transient experiments that maintain a constant temperature gradient with low liquid saturations seemed more promising than closed, steady-state systems in providing useful data to assess enhanced vapor diffusion.

\subsection{Summary}

Although many claims have been made regarding enhanced vapor diffusion, only indirect evidence exists to support EVD including the responsible mechanisms. In experiments performed in soil science, vapor flux was always inferred from other measurements such as total water flow, moisture content, and thermal conductivity/heat flux; none of the previous experiments measured the vapor flux directly. More comprehensive models of moisture transport presented in the engineering literature have been used in drying problems, but the experiments have not focused on systems where vapor-phase diffusion driven by thermal or concentration gradients is dominant.

In order to answer the question concerning the existence of EVD, mechanistic models were developed to evaluate the processes involved, and direct measurements of EVD were performed. Both of these areas were addressed in the LDRD as discussed in the next two sections. 


\subsection{PORE-SCALE ANALYSIS}

In order to try to understand the processes and mechanisms involved in enhanced vapor diffusion (EVD), pore-scale analyses have been performed. Two different types of pore-scale analysis are presented in this section; analytical and numerical. The analytical approach was initially taken to try to determine the possible significance of EVD before proceeding to a numerical approach. The numerical approach was used to investigate the mechanisms associated with EVD.

\subsection{Analytical Approach}

\subsubsection{Pore-Scale Analysis - Thermal Gradient}

A simple pore-scale analysis was performed by Ho and Webb $(1996,1998)$ to estimate the steady-state mass flow of water across liquid islands due to condensation and evaporation mechanisms caused by a thermal gradient. The geometry is depicted in Figure 3-1. A comparison of the flux "through" the liquid island (Path A-A) to the flux of water vapor caused by Fickian diffusion around the liquid island (Path B-B) is made to discern the relative importance of enhanced vapor diffusion mechanisms.

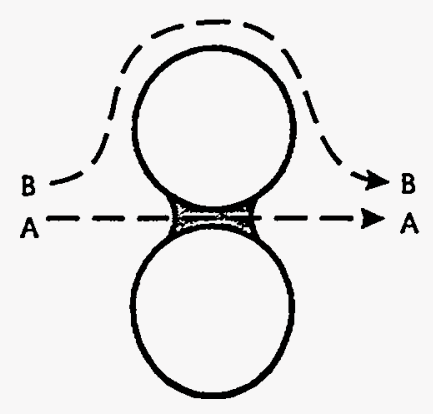

Figure 3-1

Pore-scale Transport Paths

(A-A) Enhanced Vapor Diffusion

(B-B) Fickian Diffusion

\section{Path A-A}

Consider the space between two solid particles that contains entrapped liquid water as shown in Figure 3-2. A thermal gradient in the water-wet porous medium induces vapor diffusion from the hot region (left side) to the colder regions (right side). An energy balance is performed on a control volume of the surface of the liquid island exposed to the hotter side (Figure 3-2). The latent heat of condensation on the upstream side of the liquid island is balanced by conduction through the liquid island

$$
m_{v, E V D} h_{f g}=\lambda_{l} A_{t} \frac{\Delta T}{L}
$$




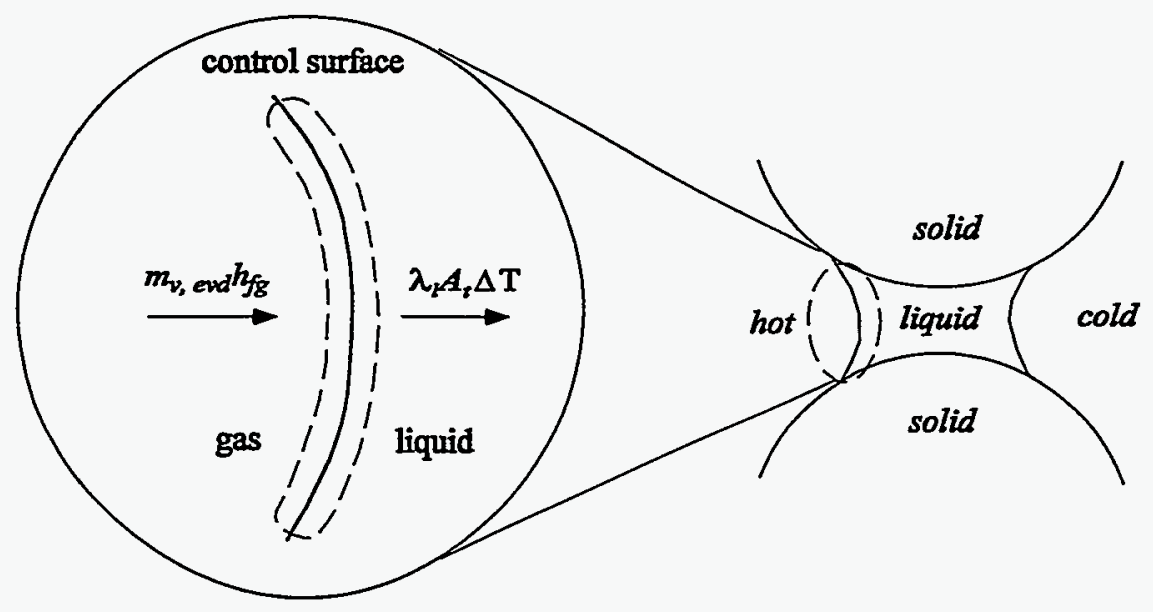

Figure 3-2

Control Surface for Energy Balance

where $m_{v, E V D}$ is the mass flow rate of vapor that condenses on the upstream end of the liquid island, $h_{f_{g}}$ is the latent heat of condensation, $\lambda_{l}$ is the liquid thermal conductivity of water, $A_{t}$ is the cross-sectional area of the surface of the liquid island, $\Delta T$ is the temperature difference across the liquid island, and $L$ is the linear distance across the liquid island. Rearranging equation (3-1) yields an expression for the mass flow of vapor due to the postulated enhanced vapor diffusion mechanisms of condensation/evaporation

$$
m_{v, E V D}=A_{t} \frac{\lambda_{l}}{h_{f g}} \frac{\Delta T}{L}
$$

\section{$\underline{\text { Path B-B }}$}

Fick's law is now used to determine the mass flow of vapor if vapor diffusion has to occur around (rather than through) the liquid island shown in Figure 3-1

$$
m_{v, F i c k}=D_{12} A_{p} \nabla C=-D_{12} A_{p} \frac{\partial C}{\partial T} \frac{\partial T}{\partial x}
$$

where $m_{v_{\text {Fick }}}$ is the mass flow rate of vapor around the liquid island due to Fickian diffusion, $D_{12}$ is the binary diffusion coefficient of air and water vapor in free space, $A_{p}$ is the cross-sectional area of pore space available for diffusion, and $C$ is the concentration (density) of water vapor. The ideal gas law is used to express the water vapor concentration, and the temperature gradient 
is expressed in terms of the temperature difference across the liquid island and the tortuosity, $\tau^{-1}$ (actual path length divided by linear path length, $L$ )

$$
m_{v, \text { Fick }}=D_{12} A_{p} \frac{M_{v}}{R T} \frac{\partial P_{v}}{\partial T} \frac{\Delta T}{\tau^{-1} L}
$$

where $M_{v}$ is the molecular weight of water $(18 \mathrm{~kg} / \mathrm{kmol}), R$ is the ideal gas constant $(8300 \mathrm{~J} / \mathrm{kmol}-\mathrm{K})$, and $P_{v}$ is the saturated vapor pressure of water.

$\underline{\text { Ratio }}$

Equation (3-2) is divided by equation (3-4) to obtain a ratio of the mass flow rates due to enhanced vapor diffusion mechanisms and Fickian diffusion:

$$
\frac{m_{v, E V D}}{m_{v, F i c k}}=\frac{A_{t}}{A_{p}} \frac{\lambda_{l}}{h_{f g} D_{12}} \frac{R T}{M_{v}} \frac{\tau^{-1}}{\frac{\partial P_{v}}{\partial T}}
$$

A value of this ratio on the order of one or more indicates that enhanced vapor phase diffusion can be significant relative to Fickian diffusion in transporting water vapor through a water-wet porous medium under a thermal gradient. Table 3-1 gives values for several parameters in equation (3-5) at $20^{\circ} \mathrm{C}$.

Table 3-1. Parameter values at $20^{\circ} \mathrm{C}$.

\begin{tabular}{|l|c|}
\hline$\lambda_{l}(\mathrm{~W} / \mathrm{m}-\mathrm{K})$ & 0.6 \\
\hline$h_{f g}(\mathrm{~J} / \mathrm{kg})$ & $2.45 \times 10^{6}$ \\
\hline$D_{I 2}\left(\mathrm{~m}^{2} / \mathrm{sec}\right)$ & $2 \times 10^{-5}$ \\
\hline$\partial \mathrm{P}_{\mathrm{v}} / \partial \mathrm{T}$ & 146 \\
\hline Viscosity (Pa.s) & $10^{-3}$ \\
\hline
\end{tabular}

Plugging the values in Table 3-1 into equation (3-5) yields the following expression:

$$
\frac{m_{v, E V D}}{m_{v, F i c k}}=11.3 \frac{A_{t}}{A_{p}} \tau^{-1}
$$


Assuming that the cross-sectional area of the liquid island and the pore space available for vapor diffusion are similar and that the tortuosity is on the order of 1 , equation (3-6) yields the following:

$$
\frac{m_{v, E V D}}{m_{v, F i c k}} \sim 10
$$

This result indicates that the contribution of water vapor transport by condensation and evaporation mechanisms across liquid islands may be significant when compared to Fickian diffusion around liquid islands.

\subsubsection{Pore-Scale Analysis - Concentration Gradient}

A similar analysis can also be performed when the conditions are isothermal and only a concentration gradient exists. The mass flow rate "through" the liquid island is driven by diffusion from the upstream boundary to the upstream end of the liquid island, and similarly from the downstream end of the liquid island to the downstream boundary. Flow in the liquid island itself is driven by the pressure difference caused by the change in curvature of the meninsci. The curvature at both ends of the liquid island will lead to a vapor pressure slightly below saturation due to vapor-pressure lowering effects; these effects are discussed in more detail later in this section. The magnitude of the vapor pressure lowering effect is on the order of $0.1 \mathrm{~Pa}$, which is small compared to the saturated vapor pressure of water of about $2337 \mathrm{~Pa}$ at $20^{\circ} \mathrm{C}$.

If vapor diffuses "through" the liquid island, and if the resistance in the liquid island is small, the flux due to EVD through the island will be much greater than around the liquid island because of the much shorter path, or

$$
m_{v, E V D}=-D_{12} A_{p} \nabla C=-D_{12} A_{p} \frac{\Delta C}{x-L}
$$

where $\mathrm{x}$ is the total distance of path A-A. The term $\mathrm{x}-\mathrm{L}$ is the vapor portion of the path A-A. The pore area is used in this case because most of the diffusion will be in the pore.

For Fickian diffusion, assuming that the distance B-B is 3 times that of A-A, the expression is

$$
m_{v, \text { Fickian }}=-D_{12} A_{p} \nabla C=-D_{12} A_{p} \frac{\Delta C}{3 x}
$$


because the diffusion occurs in a tortuous path. If the resistance of the liquid island is small, which remains to be proven, and $\mathrm{L}$ is assumed equal to $0.5 \mathrm{x}$, the ratio of diffusion mass fluxes will be

$$
\frac{m_{v, E V D}}{m_{v, F i c k}} \sim 6 .
$$

In order to bound the resistance of the liquid island, consider the pressure gradient necessary across the liquid island to support the vapor diffusion mass flux. Assume a large value of the diffusion mass flux such that the vapor pressure changes from fully saturated water vapor to completely dry over $1 \mathrm{~m}$ at $20^{\circ} \mathrm{C}$, or $0.0173 \mathrm{~kg} / \mathrm{m}^{3} / \mathrm{m}$. If EVD exists, the mass flux will be approximately that in free space, or

$$
m_{v}=-D_{12} \nabla \rho_{v}=3.7 \times 10^{-7} \frac{k g}{m^{2}-s}
$$

Assuming that the pore throat is only $10 \%$ of the total flow area, the mass flux through the pore throat becomes

$$
m_{v, \text { Pore }}=3.7 \times 10^{-6} \frac{\mathrm{kg}}{\mathrm{m}^{2}-\mathrm{s}}
$$

Using Poiseulle flow in the pore throat,

$$
\frac{d P}{d x}=-\frac{8 m_{v} \mu_{l}}{\rho_{l} r^{2}}
$$

For a viscosity value of $10^{-3} \mathrm{~Pa}-\mathrm{s}$, and a pore radius of $5 \mu \mathrm{m}$, the pressure gradient becomes

$$
\frac{d P}{d x} \sim-1.2 \frac{P a}{m} .
$$

For a liquid island length of about $50 \mu \mathrm{m}$, the pressure difference is

$$
d P_{\text {liquid island }} \sim-6 . x 10^{-5} P a .
$$


A typical pressure difference across an air-water interface is $15 \mathrm{kPa}$ for a $5 \mu \mathrm{m}$ radius of curvature. The pressure difference given by equation 3-15 is between the upstream and downstream ends of the liquid island, which translates into a change in curvature across the liquid island. Because the pressure difference is inversely related to the radius of curvature, the above pressure difference corresponds to an extremely small change in the radius of curvature $\left(\Delta \mathrm{R}_{\mathrm{c}} \sim 2 . \times 10^{-8} \mu \mathrm{m}\right)$.

\subsubsection{Discussion}

The above pore-scale analyses have qualitatively determined that EVD can exist, including the approximate magnitude. A mechanistic model of these pore-scale processes has been developed to quantify EVD processes as described in the next section. Experiments have also been conducted to confirm these results as summarized in Section 4.0.

\subsection{Numerical Approach}

A numerical, mechanistic model of the pore-scale processes involved in EVD has been developed by Webb and Ho (1997) and subsequently applied by Webb (1998a, 1999). Because of the significance of the model, the various papers referenced above have been combined in this section.

Vapor diffusion in porous media in the presence of its own liquid has often been analyzed similar to air diffusion. Air diffusion rates in porous media are much lower than in free space due to the presence of the porous medium and any liquid present. However, enhanced vapor diffusion has also been postulated such that the diffusion rate may approach free-space values. The mechanisms postulated to account for this enhancement include condensation/evaporation across isolated liquid islands in the porous media and an increased temperature gradient in the gas phase. In order to try to understand the mechanisms involved in such an enhancement, porescale models have been developed as summarized in this section. Additional details are given in Webb and Ho (1997), Webb (1998a), and Webb (1999).

Interestingly, the same phenomena of enhanced vapor diffusion due to liquid island processes has been noted in the capillary condensation literature. Due to vapor pressure lowering effects, condensation occurs in small pores in a porous media. Weisz (1975) has estimated the apparent increase in vapor diffusivity due to liquid island effects. As noted by Weisz (1975), this effect was first experimentally discovered by Carman and coworkers between 1950 and 1952 (Carmen and Malherbe, 1950, Carmen, 1952, Carmen and Raal, 1951a,b) (which is before Philip and deVries, 1957). Representative models include Lee and Hwang (1986), who developed a liquid island model for flow in a capillary, and Rajniak and Yang (1996), who proposed a pore network model for capillary condensation. However, to the authors' knowledge, a detailed investigation of the pore-scale processes such as presented in this study has not been performed. 
Pore-scale modeling of vapor diffusion in porous media is considered including the interactions of the vapor with the liquid islands. The parameters selected for this investigation are more typical of soils than for capillary condensation applications, which typically involve much smaller pores. The Dusty-Gas Model is used to simulate air-vapor advection and diffusion in a pore network including Knudsen and ordinary (Fickian) diffusion (Webb, 1996, 1998b). Surface diffusion effects are not considered. Kelvin's equation is used to estimate vapor pressure lowering effects at the liquid island/gas-liquid interface, and the Young-Laplace equation is used to evaluate gas-liquid pressure differences at either end of the liquid island. Concentration gradients are applied to the pore-scale model to calculate the vapor and air flow rates which are then compared to pure gas diffusion in the porous medium and in free space.

In somewhat similar work, Steele and Nieber (1994a,b) performed gas diffusion porescale modeling in an unsaturated medium. However, their investigation only considered gas diffusion (as opposed to vapor diffusion) and the transport of dissolved gas through stationary liquid; vapor diffusion effects were ignored. As will be evident from the results shown in this paper, vapor diffusion, along with the condensation/evaporation across liquid islands, may also influence gas diffusion. The present emphasis is on vapor diffusion, although some gas diffusion results are also presented.

In another study, Plumb and Prat (1992) evaluated the effective vapor diffusion coefficient for drying conditions using a pore-scale model. Their model calculated significant enhancement compared to gas diffusion rates, similar to enhanced vapor diffusion. However, the increase in vapor diffusion is due to evaporation from the liquid islands present in the pore-scale model. Capillary pressure and vapor pressure lowering processes were not considered, and condensation on the upstream end of the liquid island was not evaluated. This model is discussed in more detail in Prat (1993).

\subsubsection{Model Description}

In the present investigation, a rigorous pore-scale numerical model is developed to investigate the fundamental processes involved in the enhancement of vapor diffusion in porous media. Conservation of mass, flux, and energy are considered in the gas, liquid and solid phases. At the gas-liquid interface, capillary pressure due to local curvature is considered, and vapor pressure lowering is included as described below.

\subsubsection{Conceptual Model}

The conceptual model employed in the present study is depicted in Figure 3-3. The porous media is considered to be a series of randomly-arranged spheres. Heat transfer occurs between the spheres due to particle-to-particle contact, while flow of gas occurs around the spheres and around any liquid islands present. The liquid saturation is assumed to be low such that the liquid is confined to pendular rings, or "liquid islands", and no global flow of liquid 


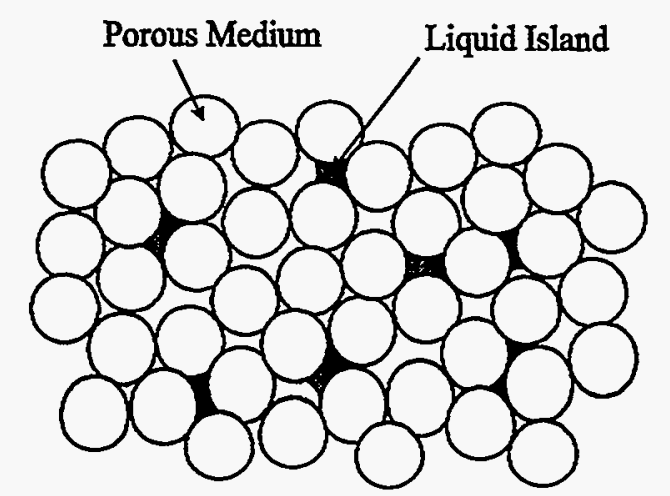

Figure 3-3

Porous Medium Conceptual Model

occurs. Gas and vapor flow due to advection and diffusion can occur due to pressure, temperature, and/or concentration gradients.

\subsubsection{Simplified Representation}

Figure 3-4 shows the simplified representation that was used in the present study based on the conceptual model shown in Figure 3-3. The particles are assumed to be arranged in rows, and the liquid islands are assumed to occur on a regular basis. Geometrical symmetry is invoked as indicated by the dashed-line box in the figure. A two-dimensional representation has been used for simplicity, and the solid particles are represented as cylinders.

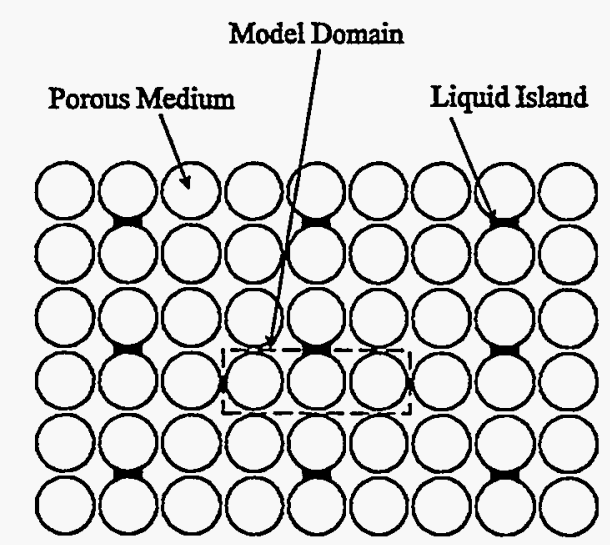

Figure 3-4

Porous Medium Simplified Representation 


\subsubsection{Numerical Method}

For the present study, the TOUGH2 (Pruess, 1991a) code has been employed. TOUGH2 is a widely-used code for simulating flow and transport in fractured and porous media. Typical applications include environmental remediation, nuclear waste isolation, and geothermal processes (Pruess, 1995, 1998). TOUGH2 is an integrated finite difference code that solves mass, flux, and energy conservation equations for fluid flow in a porous media. The formulation for an arbitrarily-shaped domain, $\mathrm{V}$, is as follows for the accumulation, flux, and source terms (Pruess and Narasimhan, 1985):

$$
\frac{d}{d t} \int_{V} M_{\mathrm{k}} d V=\int_{\Gamma} F_{\mathrm{k}} \cdot n d \Gamma+\int_{V} q_{\mathrm{k}} d V
$$

where $\kappa$ is water, air, or heat. The fluid mass accumulation term is

$$
M_{\kappa}=\phi \sum_{\beta=1}^{2} S_{\beta} \rho_{\beta} \omega_{\beta, \kappa}
$$

where $\beta$ is the liquid or the gas phase. The heat accumulation term is

$$
M_{3}=(1-\phi) \rho_{R} C_{R} T+\phi \sum_{\beta=1}^{2} S_{\beta} \rho_{\beta} u_{\beta}
$$

The flux terms for the gas and liquid phase advection, which are given below, are based on permeabilities consistent with a porous media formulation. In order to consider shear flows in the pores, the permeabilities are varied spatially to simulate a velocity distribution. This transformation is discussed later in this section.

The flux term for the gas phase is based on the Dusty Gas Model (DGM) (Mason and Malinauskas, 1983), which considers advection, ordinary diffusion, and Knudsen diffusion, including the coupling between the various mechanisms. The original version of TOUGH2 uses an advective-dispersive formulation in which the fluxes from the various mechanisms are simply added together; the coupling among the mechanisms is not considered. The difference between these two formulations is discussed in detail by Webb $(1996,1998 \mathrm{~b})$ and in Appendix A. For the DGM, the gas mass flux of component $\kappa$ in the gas phase for a binary system can be written as 


$$
\begin{aligned}
& F_{\mathrm{\kappa}}=J_{\mathrm{\kappa}} M_{\mathrm{\kappa}}=-M_{\kappa} \frac{D_{\kappa}^{K} D_{\mathrm{\kappa}} \cdot \frac{P_{g}}{R T} \nabla x_{\mathrm{\kappa}}}{D^{*}} \\
& -M_{\kappa} \frac{D_{\kappa}^{K}\left(D_{\mathrm{k \kappa}}+D_{\mathrm{\kappa}^{*}}^{K}\right) x_{\mathrm{\kappa}} \frac{\left(\nabla P_{g}-\rho_{g} g_{c}\right)}{R T}}{D^{*}} \\
& -M_{\kappa} x_{\mathrm{\kappa}} \frac{k P_{g}}{\mu_{g}} \frac{\left(\nabla P_{g}-\rho_{g} g_{c}\right)}{R T}
\end{aligned}
$$

where $\kappa=$ air or water vapor, and $\kappa^{*} \neq \kappa$, and

$$
D^{*}=D_{\mathrm{k \kappa}}+x_{\mathrm{\kappa}} D_{\mathrm{k}^{*}}^{K}+x_{\mathrm{\kappa}^{*}} D_{\mathrm{\kappa}}^{K}
$$

The above equations for the Dusty Gas Model are written in terms of mole fraction rather than mass fraction because mole fraction is a more natural variable for diffusion.

In equation 3-19, the flux of component $\kappa$ consists of a diffusive flux (first and second terms) and an advective flux (third term). The diffusive flux has ordinary diffusion (mole fraction gradient) and Knudsen diffusion (pressure gradient) components.

The flux term for the liquid phase is simply

$$
F_{l}=-\frac{k}{\mu_{l}} \rho_{l} \omega_{l, \mathrm{\kappa}}\left(\nabla P_{l}-\rho_{l} g\right)
$$

where the assumption of separation of phases, which is discussed below, has been used (relative permeability $=1$.).

The heat flux term is simply

$$
F_{3}=-K \nabla T+\sum_{\substack{\beta=1, g \\ \kappa}} h_{\beta, \mathrm{x}} F_{\beta, \mathrm{x}}
$$


Capillary pressure and vapor pressure lowering effects can be included in TOUGH2. By setting the control volume porosity equal to 0.0 or 1.0 , the control volumes can be used to represent solid or fluid regions.

TOUGH2 models elements, or control volumes, and connections between the centers of these elements. The standard version assumes that if more than one phase is present in an element, the phases are completely mixed. In the present model, the only elements which contain more than one phase are the elements at the ends of the liquid island, or the interface volumes. For simplicity, the liquid island length is defined so the interface is exactly in the middle of an interface element, and the element is half liquid and half gas. Due to capillary forces and the pore-scale nature of the present model, the phases will be separate. The flow path from the interface volume to the gas phase will be entirely gas. Similarly, the flow path between the interface element and the liquid island will be all liquid. The flow path transport parameters used in the code, such as the saturation and fluid transport properties, were modified to reflect. separate, rather than mixed, phases.

The applicability of the Dusty Gas Model to the pore scale needs to be discussed. In particular, the simple Knudsen diffusion representation of elastic molecule-wall collision breaks down as the pore dimension decreases, and Knudsen diffusion no longer applies. This diffusion regime for small dimensions is called configurational diffusion and is often encountered in diffusion through membranes. Cunningham and Williams (1980) discuss the transition between the two regimes and conclude that the approximate transition is at a capillary radius of $50 \AA$, or $0.005 \mu \mathrm{m}$. As will be seen later, the pore sizes considered in the present study are considerably larger than this transition radius, so the Dusty Gas Model is applicable. Note also that Sotirchos and Burganos (1988) have also used the Dusty Gas Model in their analysis of multicomponent gas diffusion in pore networks.

Other numerical approaches, including lattice-gas methods, could be applied to the present pore-scale problem. These methods may be more accurate than the present approach but at a cost of more model development time and effort. As discussed by Ho and Webb (1996, 1998), the existence of and the processes involved in enhanced vapor diffusion were still being evaluated when this model was developed. In fact, enhanced vapor diffusion had never been directly calculated by a mechanistic model, such as the one developed here. An approximate method, such as outlined in this paper, is an efficient approach for these initial investigations into developing a mechanistic model for EVD.

\subsubsection{Model Geometry}

Three different geometries were studied:

1) one-dimensional linear system;

2) two-dimension single pore; and

3) two-dimensional pore network. 
Table 3-2

Pore-Scale Model Parameters

Dimensions

Pore Radius

$5 \mu \mathrm{m}$

Equivalent Permeability

$2 \times 10^{-12} \mathrm{~m}^{2}$

Particle Radius

$50 \mu \mathrm{m}$

Model Porosity

0.322

Diffusion

Binary Diffusion Coefficient

$2.42 \times 10^{-5} \mathrm{~m}^{2} / \mathrm{s}$

Knudsen Diffusion Coefficient - Air $\quad 1.54 \times 10^{-3} \mathrm{~m}^{2} / \mathrm{s}$

Knudsen Diffusion Coefficient - Vapor $1.96 \times 10^{-3} \mathrm{~m}^{2} / \mathrm{s}$

The use of the one-dimensional linear system allows for a simple evaluation of the effect of the liquid island on the vapor diffusion rate, while the two-dimensional single pore includes the variation in cross-sectional area in a pore. Finally, the two-dimensional pore network considers the competition between vapor diffusion through open pores and through liquid islands. The model parameters, including the nodalization, are discussed in the next section.

\subsubsection{Model Parameters}

The model parameters are summarized in Table 3-2 and are discussed in detail below.

\subsubsection{Pore-Scale Dimensions}

The dimensions for the current pore-scale model have been chosen to be consistent with the enhanced vapor diffusion data tabulated by Jury and Letey (1979). An average value of the capillary head (pressure) data is approximately $300 \mathrm{~cm}$ (range of $<10 \mathrm{~cm}$ to $5 \times 10^{4} \mathrm{~cm}$ with a median of $269 \mathrm{~cm}$ ). Using Young-Laplace's equation for the pressure difference across a curved surface (Dullien, 1992)

$$
\Delta P=\frac{2 \sigma}{r}
$$

and an air-water surface tension, $\sigma$, at $20^{\circ} \mathrm{C}$ of $72.8 \mathrm{dynes} / \mathrm{cm}$, a typical minimum pore radius is 5 $\mathrm{x} 10^{-6} \mathrm{~m}$, or $5 \mu \mathrm{m}$.

In the present two-dimensional simulations, additional assumptions have been made in the numerical model in the application of TOUGH2. The spheres are represented by an 
octagonal geometry; an octagon was selected rather than a square in order to approximate some of the variation of the pore cross-sectional area. A non-symmetrical octagon was employed such that the faces parallel to the $\mathrm{x}$ and $\mathrm{y}$ directions have a slightly different length than the diagonal faces. This shape allows for the use of a regular grid consisting of square elements except at the diagonal faces of the solid; square elements are desirable when using the 9-point differencing scheme as discussed below. On the diagonal faces, the square elements are divided into two equal triangles, one which is solid and one which is fluid.

The particle diameter in the numerical model has been chosen to be $50 \mu \mathrm{m}$, which supports the use of square elements, and gives a reasonable model porosity value of 0.322 . An octagonal representation of a cylinder is used in the TOUGH2 model. The height and width of the octagon is $100 \mu \mathrm{m}$, consistent with the particle diameter.

The standard version of TOUGH2 employs a 5-point stencil to connect the elements in the $\mathrm{x}$ - and $\mathrm{y}$-directions. This numerical scheme is not appropriate for flow along the diagonal surfaces in the model or for flow between the square and triangular elements. Therefore, a 9point differencing scheme has been used which adds diagonal connections between elements. The main advantage of the 9-point scheme in the present model is connections parallel to the diagonal surfaces of the solid particles which allows for a more reasonable octagonal shape rather than a "stair-step" surface. As shown by Pruess and Bodvarsson (1983) and Pruess (1991b), grid orientation effects can also be significant for the 5-point scheme, especially when a diagonal surface is present; these effects are greatly reduced when a 9-point scheme is employed.

The nodalization of the three models is shown in Figure 3-5. For simplicity, the boundary elements are not shown. For the one-dimensional linear system, 24 square elements were used including boundary elements on either end of the model; the effective model length is $110 \mu \mathrm{m}$ (22 active elements x $5 \mu \mathrm{m}$ ). Making use of symmetry, the two-dimensional single-pore model is 24 elements long and 11 elements wide, and the effective model dimensions are $110 \mu \mathrm{m}$ long by $55 \mu \mathrm{m}$ wide. Finally, the two-dimensional pore network is 68 elements long and 22 elements wide. The two end columns represent boundary conditions, so the effective dimensions are 330 $\mu \mathrm{m}$ long and $110 \mu \mathrm{m}$ wide.

\subsubsection{Permeability}

The analogy between Darcy's law and laminar flow between parallel plates has been used (deMarsily, 1986), or

$$
k=\frac{r^{2}}{12}
$$

and the equivalent permeability for the minimum pore dimension of $5 \mu \mathrm{m}$ is $2 \times 10^{-12} \mathrm{~m}^{2}$. 


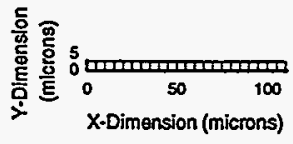

(a) One-Dimensional Linear Model

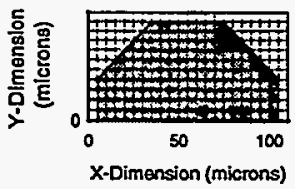

(b) Two-Dimensional Single Pore

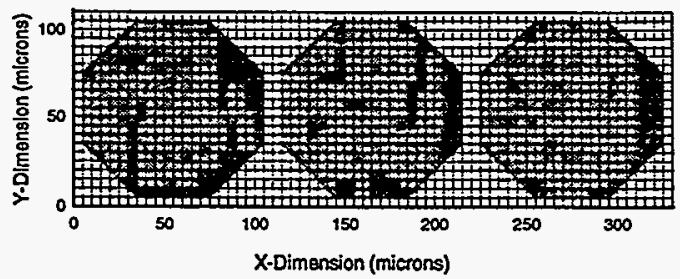

(C) Two-Dimensional Pore Network

Figure 3-5

Pore-Scale Models

The analogy between Darcy's law and laminar flow between parallel plates is based on the distance between solid surfaces and results in a parabolic velocity profile for a constant flow area channel. In the present model, the flow area varies dramatically in the direction of flow, so the suitability of a parabolic velocity profile is questionable. This problem has been at least partially addressed by Brown et al. (1995), who calculated velocity profiles between undulating surfaces of a hypothetical fracture. For the present particle geometry, the results from Brown et al. (1995) indicate that, under steady flow conditions, the fluid velocity profile will be nearly parabolic at the pore throat and "Gaussian" at the wider part of the channel. The fluid velocity is also dependent on the shape of the channel which is not captured in the analogy. Nevertheless, for simplicity, locally parabolic velocity profiles will be implicitly assumed in the present model by relying on the parallel plate analogy. Because the flow modeling is primarily concerned with diffusion, the error introduced through the use of this analogy should be small.

Accepting the parallel plate analogy for the present study, the effective permeability must vary normal to the flow direction in order to produce the desired parabolic velocity profile. The effective permeability for a given element can be derived by integrating the velocity profile over 


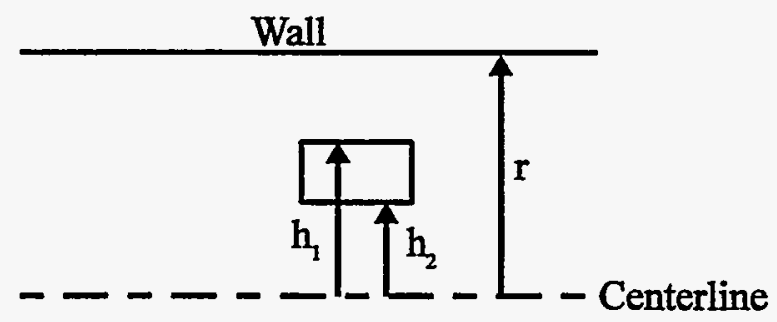

Figure 3-6

Effective Permeabilities for a Parabolic Velocity Profile

the respective coordinates as shown in Figure 3-6. For an element with coordinates $h_{1}$ and $h_{2}$ from the center of the channel to the edges of the element, the effective permeability is given by

$$
k=\frac{1}{2}\left(\frac{r^{2}}{4}-\frac{1}{12}\left(h_{2}^{2}+h_{1} h_{2}+h_{1}^{2}\right)\right) \text {. }
$$

For any given element, there may be different radii in the horizontal and vertical directions as indicated in Figure 3-7. Assuming parabolic profiles in both the horizontal and vertical directions, these radii result in different horizontal (h) and vertical (v) effective permeabilities according to the above relationship.

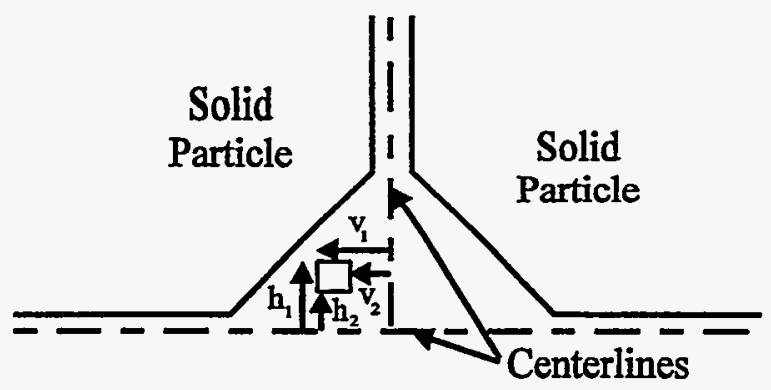

Figure 3-7

Definition of Horizontal and Vertical Effective Permeabilities

\subsubsection{Diffusion}

The binary diffusion coefficient for the present study is $2.42 \times 10^{-5} \mathrm{~m}^{2} / \mathrm{s}$ at the analysis conditions of $10^{5} \mathrm{~Pa}$ and $20^{\circ} \mathrm{C}$ (Pruess, 1991a). The Knudsen diffusion coefficient is calculated by the following formula from Cunningham and Williams (1980) for perfectly diffuse moleculewall collisions (coefficient of diffuse reflection $=1.0$ ), or 


$$
D_{x}^{K}=\frac{2}{3} r v
$$

where $v$ is the mean molecular speed

$$
v=\left(\frac{8 k_{B} T}{\pi M_{\kappa}}\right)^{1 / 2}
$$

and $k_{B}$ is Boltzmann's constant. For the present minimum pore dimension of $5 \mu \mathrm{m}$, this equation gives the value of $15.4 \mathrm{~cm}^{2} / \mathrm{s}$ or $1.54 \times 10^{-3} \mathrm{~m}^{2} / \mathrm{s}$ for air at $20^{\circ} \mathrm{C}$. For water vapor, the value for air can simply be scaled by the inverse of the square root of the ratio of molecular weights, resulting in a value of $1.96 \times 10^{-3} \mathrm{~m}^{2} / \mathrm{s}$. No modifications of the diffusion coefficients are made to account for the presence of the porous medium because the present model is a pore-scale approach.

Similar to the effective permeability discussion, any given element may have different radii in the horizontal and vertical directions. However, as discussed in detail by Cunningham and Williams (1980), the velocity profile due to Knudsen diffusion is uniform and is independent of distance from the wall. Therefore, the Knudsen diffusion coefficient is simply a function of the horizontal and vertical radii and, unlike the effective permeability, is not a function of the local coordinates.

\subsubsection{Liquid Island}

The model for the liquid island is one of the major pieces of the current pore-scale analysis. Capillary pressure across the gas-liquid interface and vapor pressure lowering is included. Capillary pressure is a function of position, or length, of the liquid island as illustrated in Figure 3-8; the capillary pressure near the minimum pore dimension (A), which corresponds to a short liquid island, is much higher than for a much larger dimension (B), or a much longer liquid island. By geometry, the radius of curvature for a given contact angle can be calculated as a function of position, or equivalently, liquid island length. Assuming a contact angle of $0^{\circ}$, the capillary pressure as a function of position is shown in Figure 3-9, where the coordinate (x) is zero at the minimum pore dimension. A maximum $x / R$ value of 0.75 , where $R$ is the particle radius, was used in the development of the capillary pressure function.

Figure 3-8

Schematic for the Liquid Island

Capillary Pressure Function

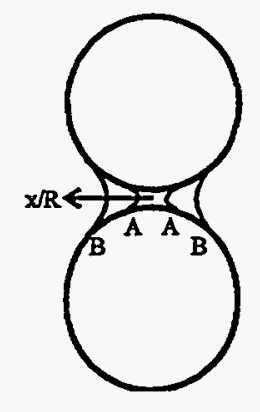




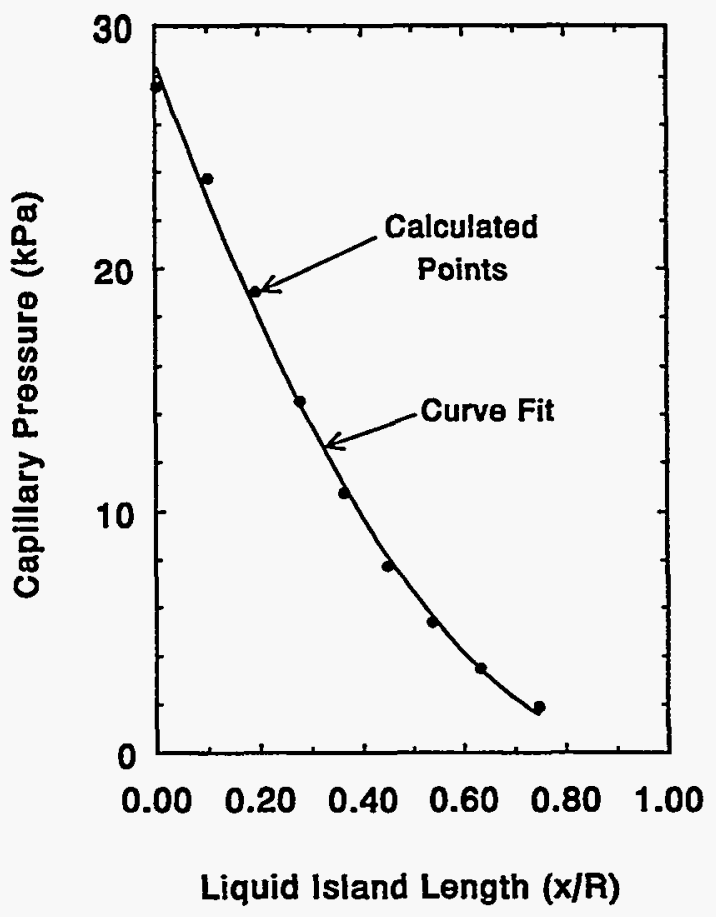

Figure 3-9

Liquid Island Capillary Pressure Function

The capillary pressure due to the gas-liquid interface results in local vapor pressure lowering due to the curvature (Dullien, 1992). This effect can be quantified through Kelvin's equation which can be written as

$$
P_{v}=f_{V P L} P_{s a t}
$$

where

$$
f_{V P L}=\exp \left(\frac{M_{w} P_{c}}{\rho_{w} R T}\right)
$$

where the capillary pressure is defined as $P_{\text {liquid }}$ minus $P_{\text {gas }}$ which is negative. For the assumed uniform temperature of $20^{\circ} \mathrm{C}$, the saturated water vapor pressure is $2337 \mathrm{~Pa}$. For the maximum capillary pressure of about $30 \mathrm{kPa}$ as shown in Figure 3-9, the vapor pressure lowering factor is 0.99978 , or a minimum vapor pressure of $2336.5 \mathrm{~Pa}$, which results in a maximum vapor pressure lowering of only about $0.5 \mathrm{~Pa}$. 
Even though the magnitude of vapor pressure lowering is small, and its effect is often ignored, it can have a large influence on enhanced vapor diffusion. For example, flow through liquid islands is driven by condensation and evaporation on both ends of the liquid island. Without vapor pressure lowering, steady-state enhanced vapor diffusion as defined in this study does not occur.

\subsubsection{Heat Conduction}

For heat conduction between touching spheres, the results of Nozad et al. (1985) have been used as corrected by Shonnard and Whitaker (1989) and Sahraoui and Kaviany (1992). A heat transfer area equivalent to $0.2 \%$ of the side of an equivalent square is used in the present study. As discussed by Shonnard and Whitaker (1989), this value is for an unconsolidated medium and is certainly not a universal parameter value. More recently, Hsu et al. (1995) investigated the appropriate contact area for the Nozad et al. data resulting in yet another estimate of $1.0 \%$. The contact area has been varied between $0.2 \%$ and $2.0 \%$ to include the range of the various investigators. Note that the effective area is highly dependent on the particle shape and contact between the particles. As discussed by Shonnard and Whitaker (1989), experiments using the same particles which had been deformed due to other uses resulted in dramatically different effective thermal conductivities. Therefore, this effective heat transfer area is highly uncertain and will obviously be much larger for consolidated medium.

\subsubsection{Boundary Conditions}

The above models (one-dimensional linear, two-dimensional single pore, twodimensional pore network) have been used in a number of investigations with different boundary conditions. Webb and Ho (1997) subjected all three models to a concentration gradient with no temperature gradient. Steady-state enhanced vapor diffusion conditions were calculated. In further studies, Webb (1998a) used the two-dimensional pore network to investigate the impact of transient boundary conditions that may occur in drying including the effect of vapor pressure lowering. Again, only a concentration gradient was imposed across the model. Finally, Webb (1999) used the two-dimensional pore network to look at the effect of a temperature gradient on the results. These studies are discussed in the next three sections.

\subsubsection{Steady-State Concentration Gradient Boundary Conditions}

The boundary conditions on the models consist of left-hand and right-hand side boundary columns with uniform conditions. A small concentration gradient is imposed across the model for the present simulations; zero pressure and temperature gradients are assumed. The objective is to calculate steady-state vapor diffusion through the various models such that there is no net change in the liquid island mass. Transient vapor diffusion, including net condensation or evaporation of liquid, is considered in the next section. 
In order to calculate steady-state vapor diffusion, the condensation and evaporation rates on the liquid island must be equal. For a zero concentration gradient, no condensation or evaporation should occur on the liquid island at equilibrium. This equilibrium condition is slightly dependent on the liquid island capillary pressure, and therefore the liquid island length, due to vapor pressure lowering effects, and the vapor pressure is slightly lower than saturation. A small concentration gradient is imposed across the model to calculate vapor diffusion; this gradient is centered around this equilibrium condition. This equilibrium condition results in slightly superheated conditions $\left(\mathrm{P}_{\mathrm{vap}}<\mathrm{P}_{\text {sat }}\right)$. The concentration gradient is sufficiently small such that the vapor pressure at the boundaries is always less than $P_{\text {sat }}$ to avoid condensation and evaporation at locations other than the liquid island. Within this limit, the results scale with the magnitude of the concentration gradient such that any enhancement is gradient independent. In all cases, a false transient was performed to achieve steady-state conditions such that mass flow rates into and out of the model were essentially the same.

The simulation results for all three models are presented below. Many of the results from the pore-scale model are compared to the free-space gas diffusion results calculated by Fick's law, or

$$
F_{i, \text { Fickian diffusion }}=-D_{12} \rho_{g} \nabla \omega_{i}
$$

with an air-water vapor density of $1.178 \mathrm{~kg} / \mathrm{m}^{3}$ and the binary diffusion coefficient as listed in Table 3-2. For a binary air-water vapor system, the diffusive mass fluxes according to Fick's law will be equal and opposite. The assumption of equal and opposite mass fluxes in a binary system is inconsistent with Graham's law of diffusion as discussed later.

\subsection{One-dimensional Linear System}

A one-dimensional linear model based on the minimum pore dimension has been used as the initial model investigating enhanced vapor diffusion as depicted earlier in Figure 3-5, A liquid island is centered in the one-dimensional model, and the appropriate boundary conditions are imposed. Because the flow area is uniform, the capillary pressure is constant, and there is no unique liquid island position. In this case, there is also a stability concern, since any change in the liquid island length has no effect on the liquid island capillary pressure or vapor pressure lowering. In order to keep the liquid island in the center of the linear model, and to allow feedback between the liquid island length and condensation/evaporation processes, the twodimensional capillary pressure curve shown earlier in Figure 3-9 was used. 


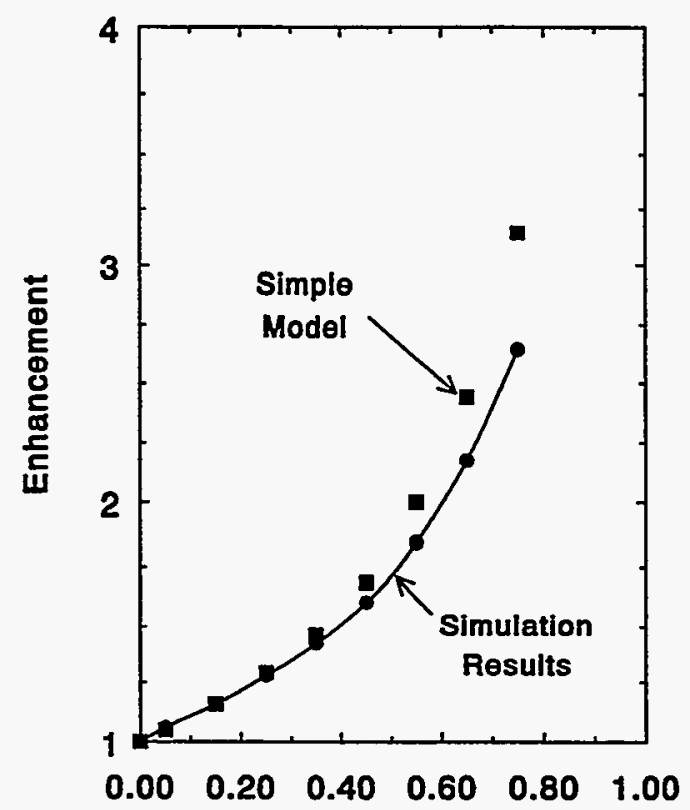

Liquid Island Length $(x / R)$

Figure 3-10

Enhancement of Vapor Diffusion for the One-Dimensional Linear Model

When there is no liquid island, pure gas diffusion occurs. The present simulations use the Dusty Gas Model which is more appropriate to a porous medium than Fick's law as discussed by Webb $(1996,1998 \mathrm{~b})$ and in Appendix A. For Fick's law, the water vapor and air diffusive mass fluxes are equal and opposite. However, in porous media, Graham's law of diffusion applies, and the diffusive mass fluxes will be proportional to the square root of the ratio of the molecular weights (Mason and Malinauskas, 1983). (Also note that the mole fluxes are not equal). For the one-dimensional linear model, the vapor diffusive mass flux is $2 \%$ lower than Fick's law, while the air diffusive mass flux is $25 \%$ higher than Fick's law; this difference is consistent with the ratio predicted by Webb (1998b). As a result of the unequal diffusive mass fluxes, the porous media factors will be different for water vapor and for air.

The enhancement in vapor diffusion as a function of liquid island length is given in Figure 3-10. Enhancement is defined as the vapor diffusion rate compared to the all-gas case, or $\eta$. As shown in the figure, vapor diffusion may be significantly enhanced in the presence of a liquid island. The longer the liquid island, the greater the enhancement. 
Also shown in Figure 3-10 is a function which is simply proportional to the length of the vapor diffusion path in the model, assuming that the liquid island has zero resistance (note that the total model length is $110 \mu \mathrm{m}$ whereas the particle diameter is $100 \mu \mathrm{m}$ ). The shortened diffusion path length model overestimates the enhancement given by the simulations because the liquid island resistance is neglected.

\subsection{Two-dimensional Single Pore}

Figure 3-5 shows the two-dimensional single pore model which includes variation in cross-sectional area in a pore. Initial results with no liquid island (all gas) indicate that the porous media factor, $\beta$, for water vapor is 0.147 compared to Fick's law value in free space. As mentioned above, the porous media factor for air will be different than for water vapor and is 0.186 for the all-gas case. These porous media factors (air and water vapor) are reasonably consistent with the theoretical value predicted by Ryan et al. (1981) of approximately 0.17 at the model porosity and with the experimental data shown by them for unconsolidated media.

Figure 3-11 plots the enhancement in vapor diffusion for the two-dimensional single pore model. Similar to the one-dimensional linear results, vapor diffusion is significantly enhanced when a liquid island is present and increases with liquid island length. The "kink" in the results

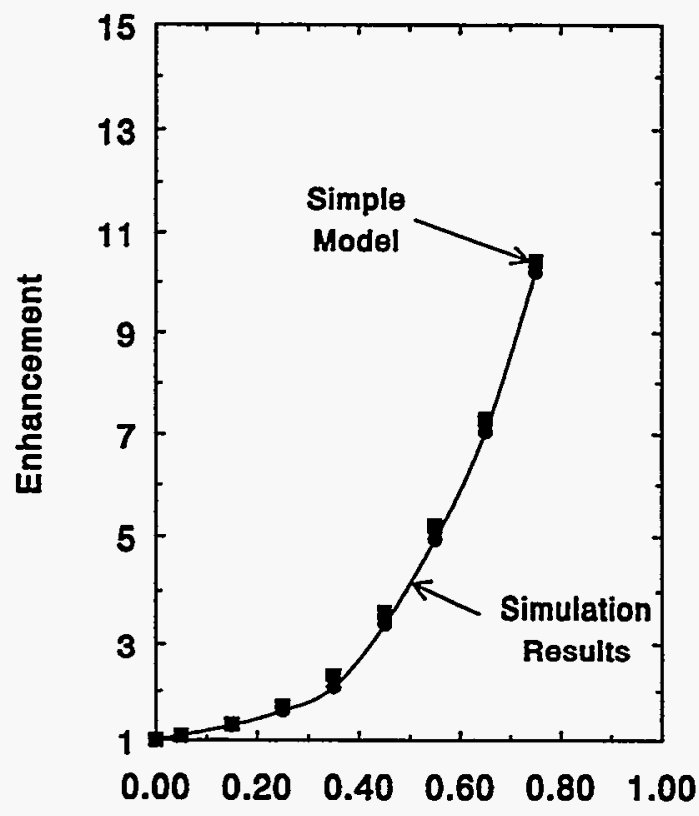

Liquid Island Length ( $x / R)$

Figure 3-11

Enhancement of Vapor Diffusion

for the Two-Dimensional Single Pore Model 
at a liquid island fractional length of 0.40 is due to the change in the model pore flow area at that location caused by the octagonal representation of the solid particles. In the present case, the enhancement is up to an order of magnitude for the longest liquid island considered. Simplified model results are also shown in Figure 3-11. The model simply integrates the diffusion equation over the gas portion of the model including the variation in pore area, assuming that the liquid island portion has zero resistance. This simplified model agrees very well with the simulations.

Figure 3-12 shows the porous media factor, $\beta$, for vapor diffusion. The value increases from the all-gas value of 0.147 to a maximum value of 1.5 , or $50 \%$ larger than the free-space value. Recall that Jury and Letey (1979) estimated that the porous media factor would be approximately 1.0. Because this model only considers a single pore, there is no diffusion of gas from one boundary to the other (dissolved gas diffusion is not included). Air diffusion results will be presented for the two-dimensional pore network which is discussed next.

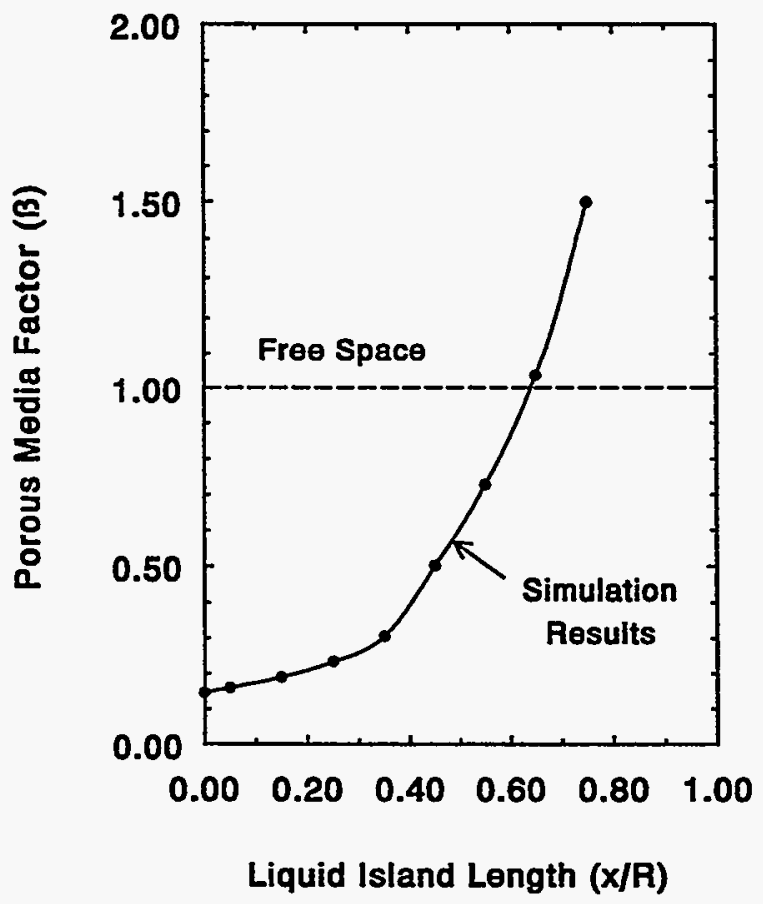

Figure 3-12

Vapor Diffusion Porous Media Factor

for the Two-Dimensional Single Pore Model 


\subsection{Two-dimensional Pore Network}

The two-dimensional pore network is the most general of the three models described in this study and is intended to investigate the competition between open pores and liquid islands. In this model, only a single liquid island is introduced, so any overall enhancement will be small. In addition to the competition for vapor diffusion between the liquid island and open pores, this model addresses diffusion of the gas phase (air) in the presence of a liquid island.

The case for gas-only diffusion, or diffusion without liquid islands, gives results identical to the two-dimensional single pore for the porous media factor. Because the boundary conditions specified zero pressure gradient, all flow is due to diffusion. The mass flow vectors for water vapor and air are given in Figure 3-13. Because of the concentration gradient imposed, vapor diffuses from right-to-left, while air diffuses from left-to-right. The mass flux of air is higher than the mass flux of water vapor consistent with Graham's law of diffusion as discussed earlier.

For cases with a liquid island, even though the boundaries have the same total pressure, advection and Knudsen diffusion occur. While vapor essentially flows "through" the liquid island, gas (air) is effectively blocked by the liquid island and builds up on the upstream end.

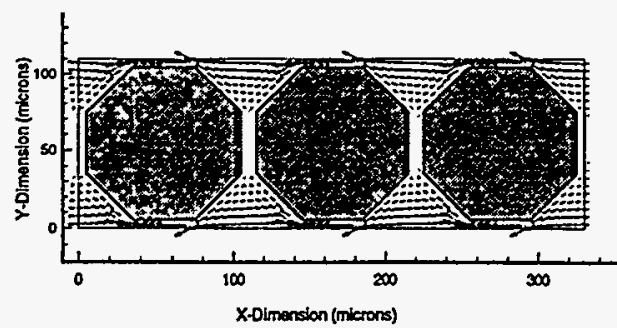

(a) Water Vapor Total Mass Flow Vectors

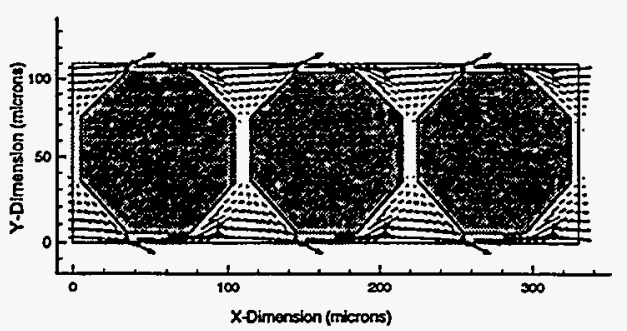

(b) Air Total Mass Flow Vectors

Figure 3-13

Mass Flow Vectors for the Two-Dimensional Pore Network Model For All Gas Conditions 


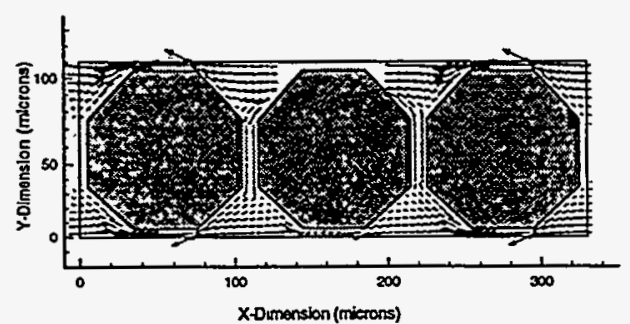

(a) Water Vapor Total Mass Flow Vectors

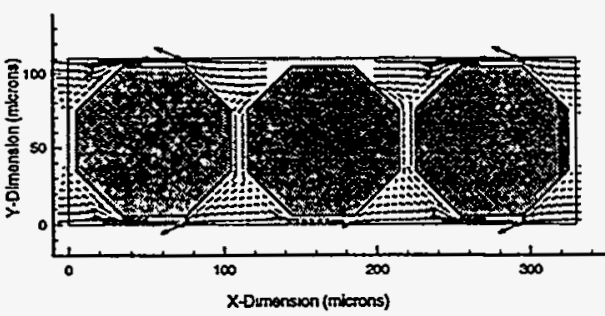

(b) Water Vapor Diffusion Mass Flow Vectors

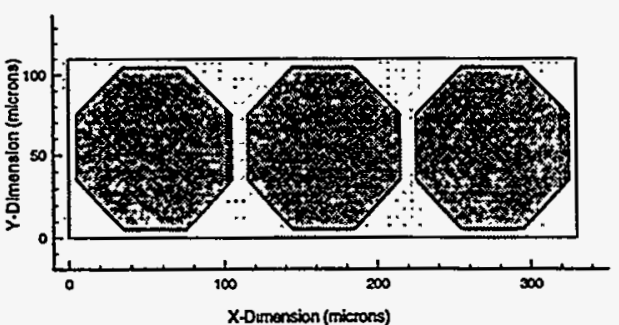

(c) Water Vapor Advection Mass Flow Vectors

Figure 3-14

Water Vapor Mass Flow Vectors for the Two-Dimensional Pore Network Model For a Liquid Island Length of $x / R=0.75$

Because air is stagnant at the liquid island, diffusion away from the liquid island caused by the buildup of air must be balanced by advection of air (and water vapor) towards the liquid island. Therefore, there are diffusion, advection, and Knudsen diffusion contributions to vapor and air flow.

Figures 3-14 and 3-15 show the mass flow vectors for water vapor and air in the case of the longest liquid island, respectively. The liquid island is assumed to be in the top pore throat of the center particle. Three velocity vectors plots are shown for the total, diffusive, and advective mass velocity contributions using the same scale. Knudsen mass velocity fluxes are not shown since their contribution is small in the present simulations. Vapor essentially diffuses "through" the liquid island via condensation/evaporation mechanisms as indicated by the vector plots. The total vapor mass flow is dominated by diffusive fluxes; the advective contribution is small as indicated by the vector plots. Upstream, the liquid island "pulls" vapor from the open-pore side of the model, even though this vapor flow path is considerably longer than if the vapor flowed 


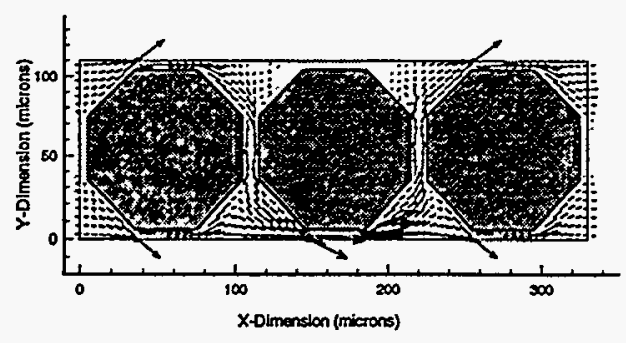

(a) Air Total Mass Flow Vectors

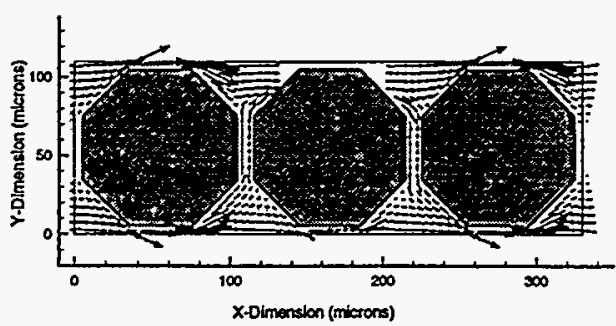

(b) Air Diffusion Mass Flow Vectors

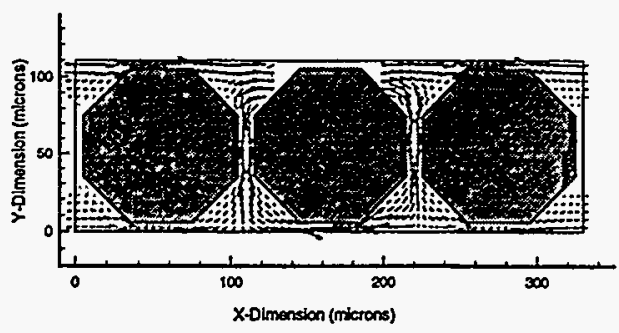

(C) Air Advection Mass Flow Vectors

Figure 3-15

Air Mass Flow Vectors for the Two-Dimensional Pore Network Model For a Liquid Island Length of $\mathrm{x} / \mathrm{R}=0.75$

straight through. On the downstream portion, the vapor flux from the liquid island is "pushed" back to the open-pore side. The vapor mass velocity vectors are slightly larger than for the allgas case indicating enhanced vapor diffusion. For the air, the flow pattern is just about the opposite of the vapor. Since the liquid island effectively blocks air flow, the air must go around the liquid island, and air is stagnant next to the interface. At the right end of the liquid island, vapor diffuses toward the gas-liquid interface; therefore, air diffusion will be opposite and away from the interface. Because air is stagnant, a small pressure gradient will be established to balance the air diffusive flow rate. This pattern is seen in the velocity vectors. There is a significant advective air flow rate in the middle of the model, especially near the liquid island.

The water vapor concentration profiles in the $\mathrm{x}$-direction in the top and bottom rows are shown in Figure 3-16; the water vapor mass fractions are normalized to the difference across the model. For the all-gas case, the top and bottom row conditions are identical due to symmetry. The gradient is smaller in the open portions of the pores and increases dramatically in the pore throats as expected. When the liquid island is present, the profiles are significantly different. For 
the bottom row with the open pore, the gradient is larger than the all-gas case in the first and last pores, indicating that the liquid island increases vapor diffusion through open pores compared to the all-gas case. In the middle pore, which is opposite the liquid island, the gradient is significantly smaller than the all-gas gradient, indicating that significant flow is diverted to the liquid island side. For the liquid island row, the gradient and the vapor diffusion rate is higher than the other cases. The profile is "missing" in the location of the liquid island because there is no vapor. The vapor mass fraction is practically the same on either end of the liquid island, indicating that the water vapor essentially diffuses "through" the liquid island with minimal resistance. These profiles indicate that not only does vapor diffuse "through" the liquid island, but that the liquid island enhances the diffusive flow in open pores, which is also indicated in the mass flux vectors in Figure 3-14.

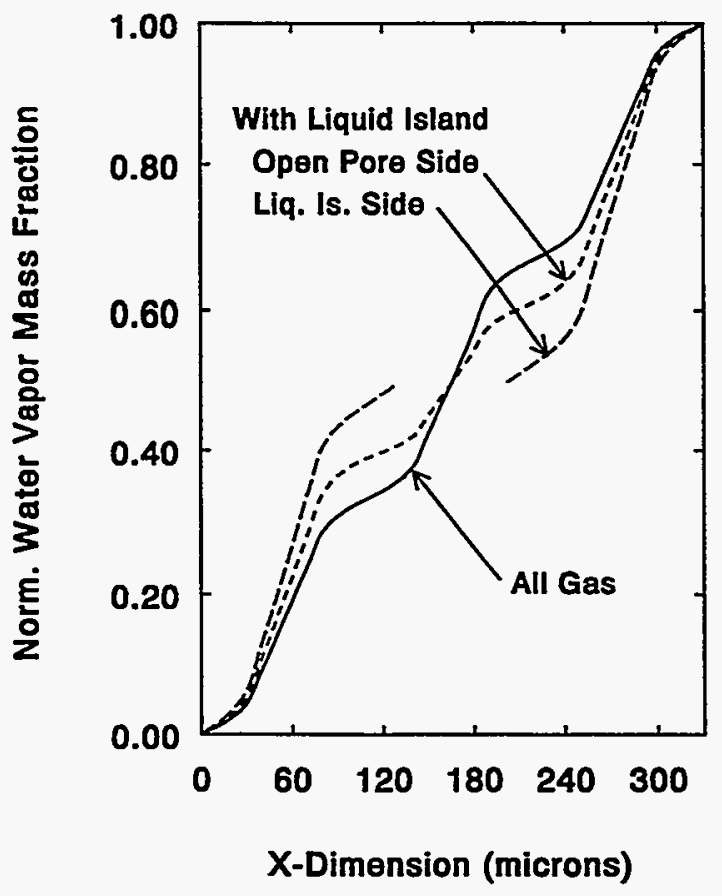

Figure 3-16

Normalized Water Vapor Mass Fraction Profiles

The vapor diffusion enhancement for the pore network with a single liquid island is given in Figure 3-17; the reason for the "kink" in the results at a liquid island length of 0.4 is due to the model pore flow area variation discussed before. For the present results, all flow mechanisms (diffusion plus advection plus Knudsen diffusion) are lumped together into a total "apparent" diffusive flow. The maximum enhancement is only about $30 \%$ (compared to over a factor of 10 for the single pore) because only one liquid island is specified. The competition between a liquid island and an open pore is the primary purpose of this model. Again, the simple model gives reasonable results. 


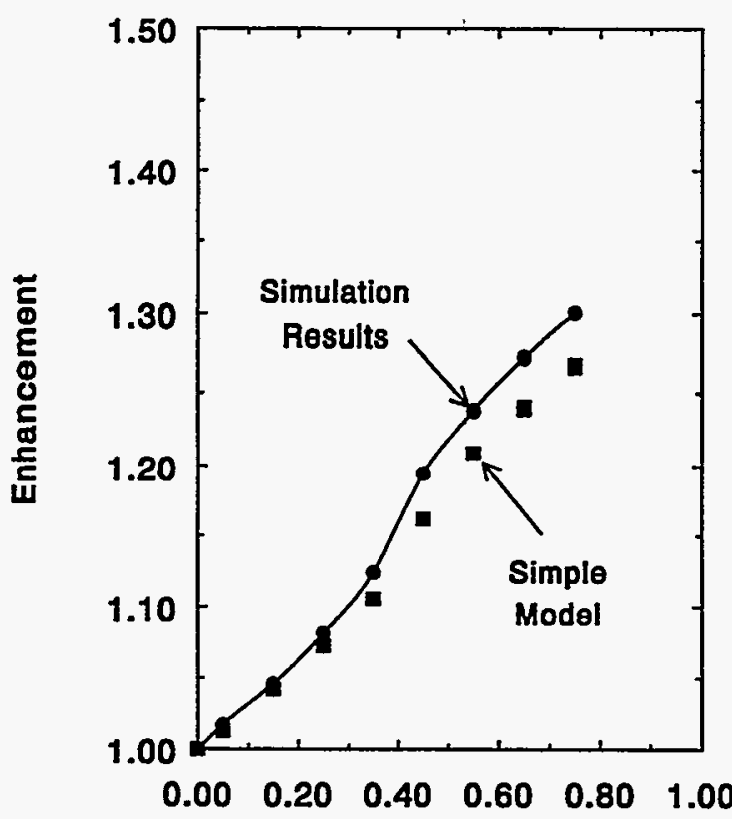

Liquid Island Length $(x / R)$

Figure 3-17

Enhancement of Vapor Diffusion

for the Two-Dimensional Single Pore Model

In contrast to vapor diffusion, which is enhanced by the presence of a liquid island, the diffusion of air is reduced with the introduction of a liquid island, probably due to the blocking effect of the liquid island which increases the path length for the flow of air. The porous media factor drops from 0.186 for the all-gas case to 0.136 with the shortest liquid island. The air porous media factor increases slightly as the liquid island length increases to 0.141 for the longest liquid island. The water vapor and air porous media factors as a function of liquid island length are shown in Figure 3-18 for the two-dimensional pore network.

\subsection{Discussion}

The results from the present pore-scale modeling investigation indicate that significant enhancement of vapor diffusion in porous media is indeed possible. The one-dimensional linear and two-dimensional pore models support the possibility of significant enhancement. Enhanced vapor diffusion in porous media may be comparable to free-space values. The two-dimensional pore network model supports the idea that vapor may preferentially flow through liquid islands rather than through open pores. Vapor is diverted from an open pore to a pore with a liquid island. 


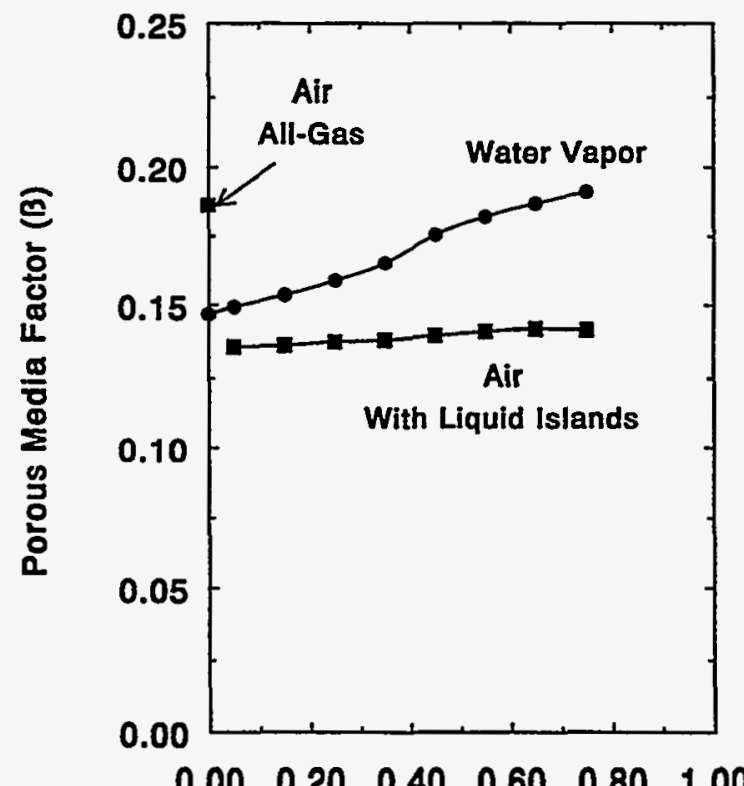

Liquid Island Length $(x / R)$

Figure 3-18

Water Vapor and Air Porous Media Factors

for the Two-Dimensional Pore Network Model

In all three models discussed above, the liquid flow rate in the liquid island is equal to the vapor diffusion rate, resulting in steady-state conditions. The driving force for the liquid flow is the difference in capillary pressure between the condensing and evaporation interfaces. The location of the interfaces, and the resulting capillary pressure, is calculated by the model based on the condensation/evaporation rate and flow to and from the interfaces.

The present results represent the first known mechanistic calculation of enhanced vapor diffusion in porous media. Based on the present results, enhanced vapor diffusion is possible or even probable. The enthusiasm for the present simulation results must be tempered with reality. While the present pore-scale model indicates that enhanced vapor diffusion is possible, only experimental data can confirm and quantify the process.

In many current engineering studies, gas and vapor diffusion are treated similarly, often using Fick's law. In addition to problems with Fick's law as discussed earlier, if enhanced vapor diffusion exists, gas and vapor diffusion should be treated differently in the presence of liquid. While gas diffusion is reduced due to the liquid, vapor diffusion may be considerably increased. 


\subsection{Conclusions}

Three pore-scale models of enhanced vapor diffusion in porous media (one-dimensional linear, two-dimensional single pore, and a small two-dimensional pore network) have been developed. The conclusions of the present investigation are:

1. Significant enhancement of vapor diffusion is possible in the presence of liquid islands. The enhancement increases dramatically as the length of the liquid island increased.

2. Vapor diffusion rates in pore-scale models may approach those in free space due to liquid island effects.

3. Gas and vapor diffusion should be treated differently. Gas diffusion decreases slightly in the presence of liquid islands, while vapor diffusion may be considerably enhanced.

4. A simple diffusion model assuming that the liquid island has zero resistance to diffusion gives reasonable results compared to detailed simulations.

\subsubsection{Transient Boundary Conditions - Drying}

Transient boundary conditions have been applied to the above two-dimensional pore network model in order to simulate drying conditions. For these simulations, only the longest liquid island is considered $(x / R=0.75)$. The resulting vapor pressure lowering (VPL) is only about $0.033 \mathrm{~Pa}$, or $0.0014 \%$ of the saturated value. Even though the magnitude of vapor pressure lowering is small in magnitude and its effect is often ignored, it can have a large influence on enhanced vapor diffusion. For example, flow through liquid islands is driven by condensation and evaporation on both ends of the liquid island. Note that without vapor pressure lowering, steady-state enhanced vapor diffusion does not occur under isothermal conditions as discussed above.

The boundary conditions consist of left-hand and right-hand side boundary columns with uniform conditions in each column as above. For steady-state vapor diffusion in which the flow rate into and out of the model are equal, the condensation and evaporation rates on the liquid island must be the same. A small concentration gradient, which is centered around this equilibrium condition, is then imposed to calculate steady-state vapor diffusion. As the concentration gradient is increased, the higher vapor-pressure (higher relative humidity (RH)) boundary is limited by the saturation vapor pressure; this condition is the limit of steady-state vapor diffusion. In order to increase the gradient further, the lower vapor-pressure (lower $\mathrm{RH}$ ) boundary value is decreased resulting in transient drying boundary conditions. In this case, net evaporation occurs from the liquid island, and the mass flow rate into and out of the model are not equal. In the present calculations, the lower vapor-pressure boundary was reduced all the way to a zero vapor pressure (zero RH) condition. As will be seen, this combination of steadystate and transient drying calculations results in about a five to six order-of-magnitude variation in the vapor pressure $(\mathrm{RH})$ gradient across the model. 
The enhancement factor for vapor diffusion is defined as the calculated vapor flux with a liquid island divided by the vapor flux for all-gas conditions. The vapor flux for all-gas

conditions is easily calculated by simply removing the liquid island. Similarly, for a liquid island under steady-state boundary conditions, the vapor fluxes into and out of the model are the same, and the enhancement factor is simply the vapor flux divided by the all-gas flux for the same boundary conditions.

However, for transient drying conditions, the situation is more complicated. The vapor flux is spatially variable, i.e., the vapor flux into the model is not equal to the vapor flux out of the model due to net liquid island evaporation. For the purposes of defining an average enhancement factor, the average of the mass flux into and out of the model is used. As will be seen, this definition has many advantages.

The basic model used in the present study was shown earlier in Figure 3-5c. A liquid island is located in the top center throat of the model. As mentioned above, with vapor pressure lowering, the equilibrium vapor pressure at either end of the liquid island is only reduced by about $0.033 \mathrm{~Pa}$, resulting in an equilibrium relative humidity of 99.9986 percent.

Both steady-state and transient conditions are considered. Steady-state occurs when the boundary conditions are centered around the liquid island condition such that condensation and evaporation rates are equal. The range of boundary relative humidities is dramatically limited for this situation such that the minimum relative humidity $(\mathrm{RH})$ value of the lower vapor pressure boundary is about 99.9972 percent (with a higher vapor pressure boundary condition RH of 100 percent). For transient conditions, in which the condensation and evaporation rates are not equal, the lower $\mathrm{RH}$ boundary can decrease all the way to 0 . This boundary condition imposes a severe vapor pressure gradient which results in strong evaporation from both ends of the liquid island.

\subsection{With Vapor Pressure Lowering}

Steady-state vapor diffusion results for the vapor velocity vectors are shown in Figure 319 for all-gas conditions and for a liquid island with two different RH differences. The liquid island is located in the top pore of the center particle. The velocity vectors are normalized with respect to the $\mathrm{RH}$ difference to facilitate comparisons. The plots are practically identical for the two RH differences shown and indicate that vapor diffuses "through" the liquid island. More vapor diffuses through the liquid island than through the open pore on the opposite side of the center particle even though the pore geometries are the same. This behavior is caused by the liquid island which reduces the effective diffusion path for the vapor and actually "pulls" vapor towards it. As the RH difference is increased further, transient vapor diffusion occurs, and there is net evaporation from the liquid island as shown in the vector plots in Figure 3-20. These velocity vectors are different than for steady-state due to the significant net evaporation from the liquid island, especially at the downstream end. Eventually, there is evaporation from both ends of the liquid island due to the strong vapor pressure gradient. 


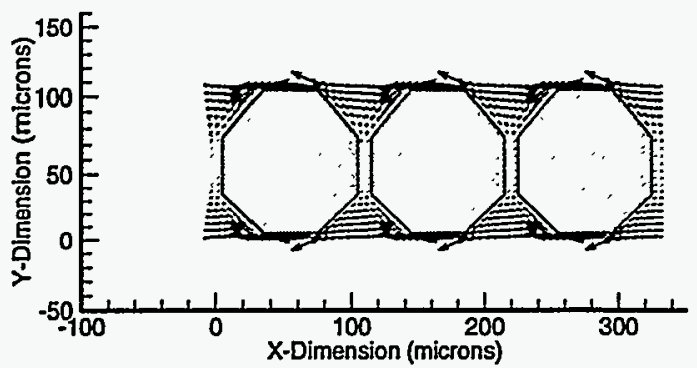

(a) All-Gas Conditions - $\triangle \mathrm{PH}=0.001 \%$

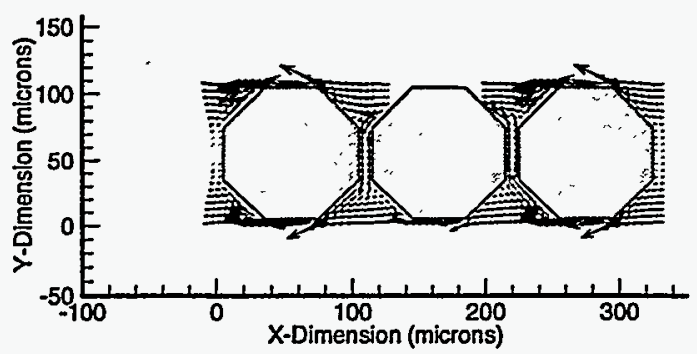

(b) With Liquid Island - $\triangle \mathrm{RH}=0.001 \%$

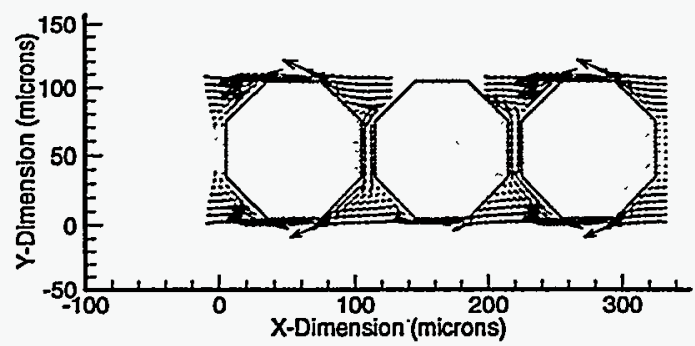

(c) With Liquid Island - $\triangle \mathrm{RH}=0.002 \%$

Figure 3-19

Velocity Vectors with VPL for Steady-State Boundary Conditions

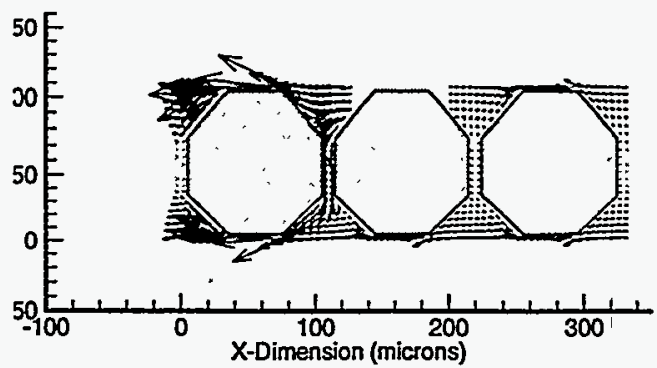

(a) With Liquid Island - $\triangle \mathrm{RH}=0.006 \%$

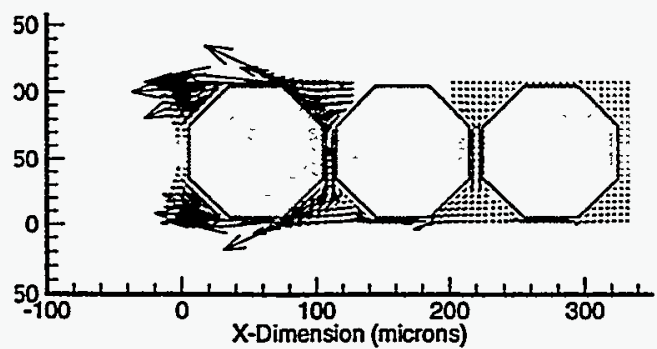

(b) With Liquid Island - $\Delta \mathrm{RH}=0.1 \%$

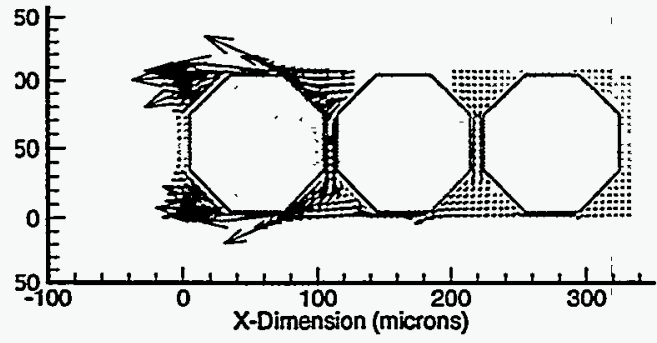

(c) With Liquid island - $\Delta \mathrm{RH}=100 \%$

Figure 3-20

Velocity Vectors with VPL for Transient Conditions

The results from the various simulations can be summarized by plotting the enhancement of the vapor mass flux across each boundary as well as the average value as depicted in Figure 321. The enhancement value is defined as the vapor mass flux across a boundary divided by the vapor mass flux across the boundary for all-gas conditions with the same boundary conditions. For steady-state boundary conditions, the vapor mass flux across the lower and higher $\mathrm{RH}$ boundaries is the same by definition, and the value of the enhancement factor is constant at about 1.3. (Note that if more liquid islands were present, the enhancement factor would be much 


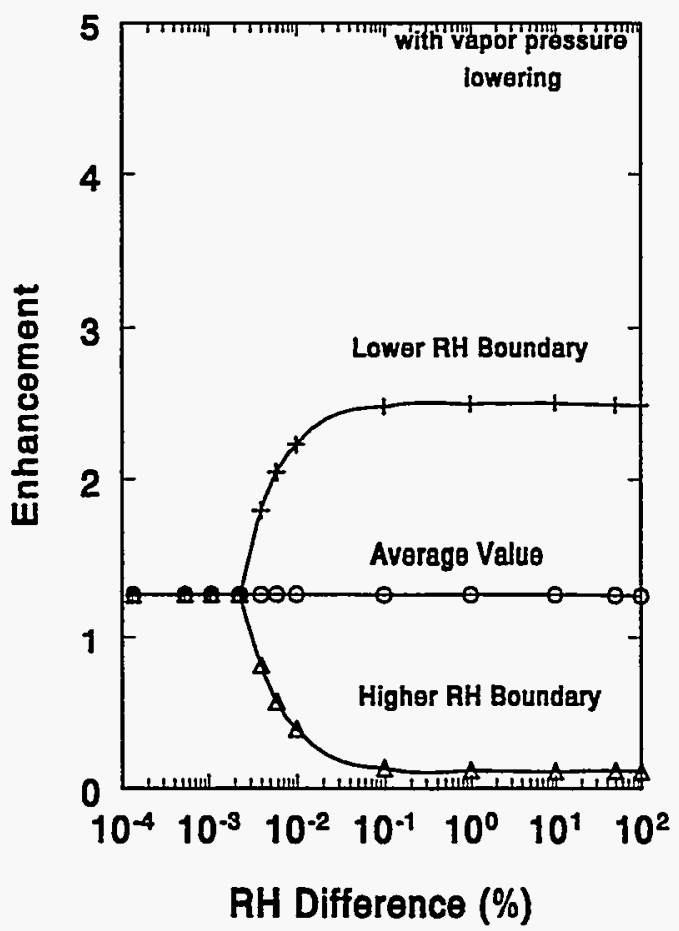

Figure 3-21

Enhancement Factors with Vapor Pressure Lowering

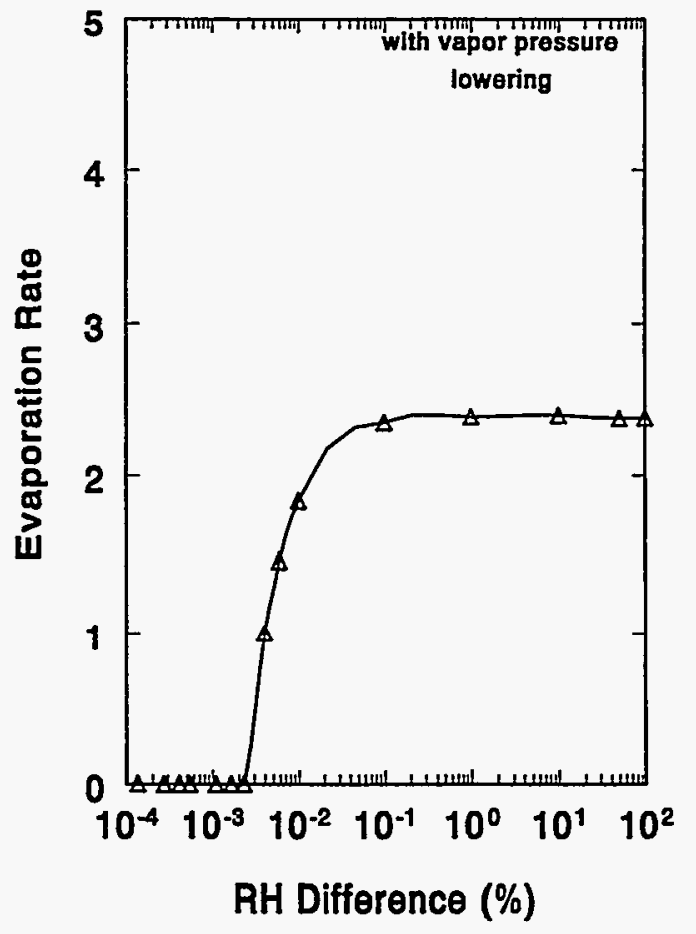

Figure 3-22

Evaporation Rate

With Vapor Pressure Lowering

larger. For example, Webb and Ho (1997) calculated enhancement factors greater than 10.) With transient boundary conditions, the enhancement factors for each boundary change, but somewhat surprisingly, the average value remains constant and equal to the steady-state boundary results. Therefore, based on these simulations, enhancement factors based on the average vapor mass flux are the same for steady-state and transient conditions. These results imply that the enhancement factor data obtained by Gu et al. (1998) were not affected by the transient nature of the experiments as long as the average vapor mass flux is used.

The influence of the boundary conditions on the net evaporation rate from the liquid island is important in determining the liquid island evolution for transient conditions. If net evaporation occurs, the liquid island will disappear with time. Figure 3-22 shows the net liquid island evaporation rate (mass flux to lower RH boundary minus mass flux from higher RH boundary) normalized to the all-gas diffusion rate. For steady-state conditions, no net evaporation occurs, so any liquid island would persist. The vapor mass flux from the higher $\mathrm{RH}$ boundary and the mass flux to the lower RH boundary are equal. As indicated by the velocity vector plots, a significant amount of vapor "diffuses" through the liquid island. As the RH difference is increased into the transient regime, net evaporation increases considerably such that most of the flow is due to evaporation of the liquid island. 


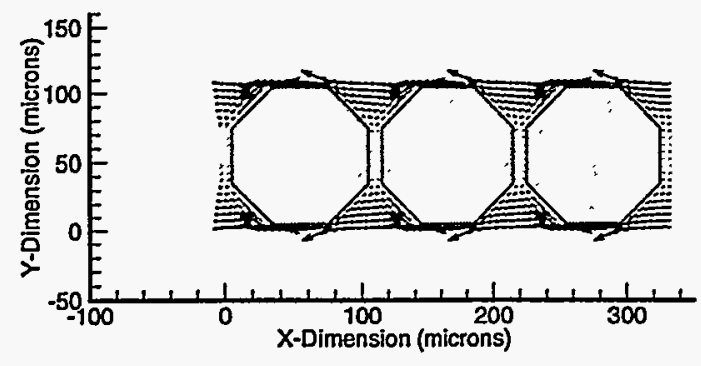

(a) All-Gas Conditions - $\triangle \mathrm{RH}=0.001 \%$

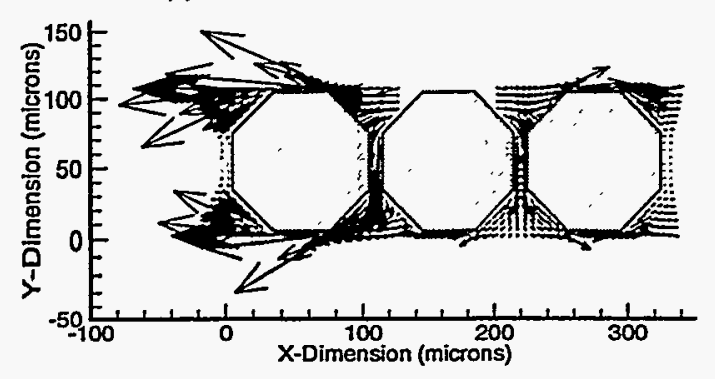

(b) With Liquid lsland - $\Delta \mathrm{RH}=0.001 \%$

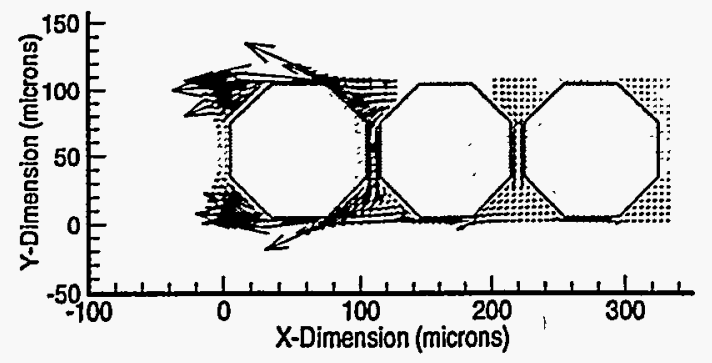

(c) With Liquid Island - $\triangle \mathrm{RH}=0.002 \%$

Figure 3-23

Velocity Vectors without VPL for Steady-State Boundary Conditions

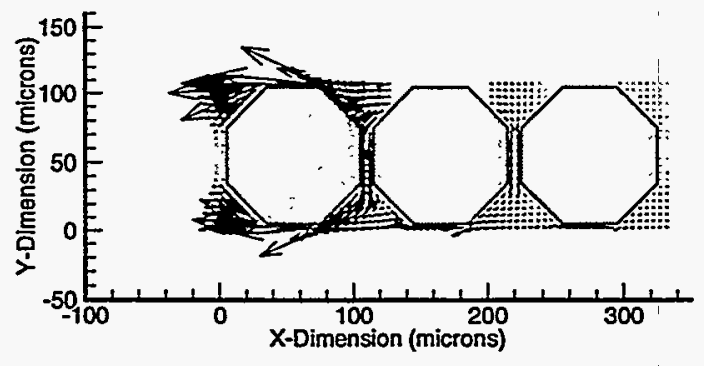

(a) With Liquid Island - $\triangle \mathrm{RH}=0.006 \%$

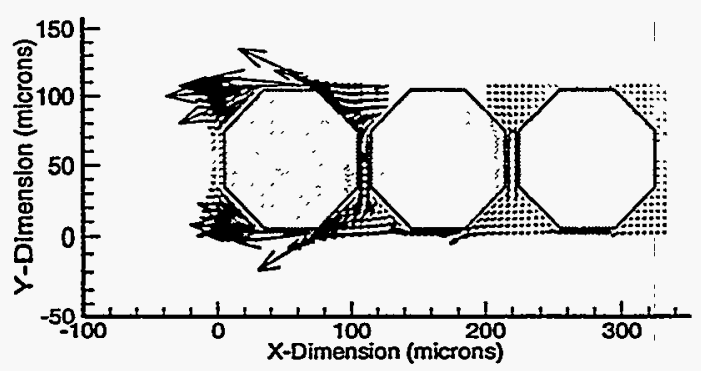

(b) With Liquid island - $\triangle \mathrm{RH}=0.1 \%$

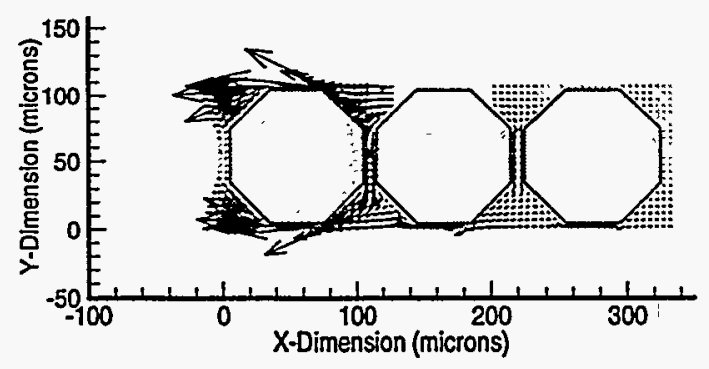

(c) With Liquid Island - $\triangle \mathrm{RH}=100 \%$

Figure 3-24

Velocity Vectors without VPL for Transient Boundary Conditions

\subsection{Without Vapor Pressure Lowering}

Vapor pressure lowering is often ignored in pore-scale models. Vapor diffusion results for the vapor velocity vectors are shown in Figure 3-23 without vapor pressure lowering (VPL) for the same conditions as Figure 3-19. As mentioned earlier, steady-state conditions do not exist without vapor pressure lowering. For the lower $\mathrm{RH}$ difference, evaporation occurs from both 
ends of the liquid island. As the RH difference increases, evaporation shifts toward the lower $\mathrm{RH}$ end of the liquid island. (With vapor pressure lowering, net evaporation did not occur for these RH differences.) For transient boundary conditions, the velocity vectors given in Figure 324 indicate that evaporation still occurs predominantly from the lower RH end of the liquid island. The results for the larger RH differences are practically identical to those with vapor pressure lowering. In summary, for steady-state and the lower RH difference transient boundary conditions, the results are significantly different with and without vapor pressure lowering. For transient conditions at the higher RH differences, the results with and without vapor pressure lowering converge.

The enhancement factors from the various simulations without vapor pressure lowering are shown in Figure 3-25; the factors with vapor pressure lowering are also included for comparison purposes. While the average value of the enhancement factor is constant and is the same with or without vapor pressure lowering, the behavior of the boundary enhancement factors differs dramatically. For steady-state conditions without vapor pressure lowering, the enhancement factor is negative for small RH differences indicating vapor flow toward the higher $\mathrm{RH}$ boundary. The RH of the liquid island is 1.0 because vapor pressure lowering is neglected. For transient conditions, the enhancement factors are independent of $\mathrm{RH}$ difference. At the higher RH differences, the enhancement factors with and without vapor pressure lowering converge.

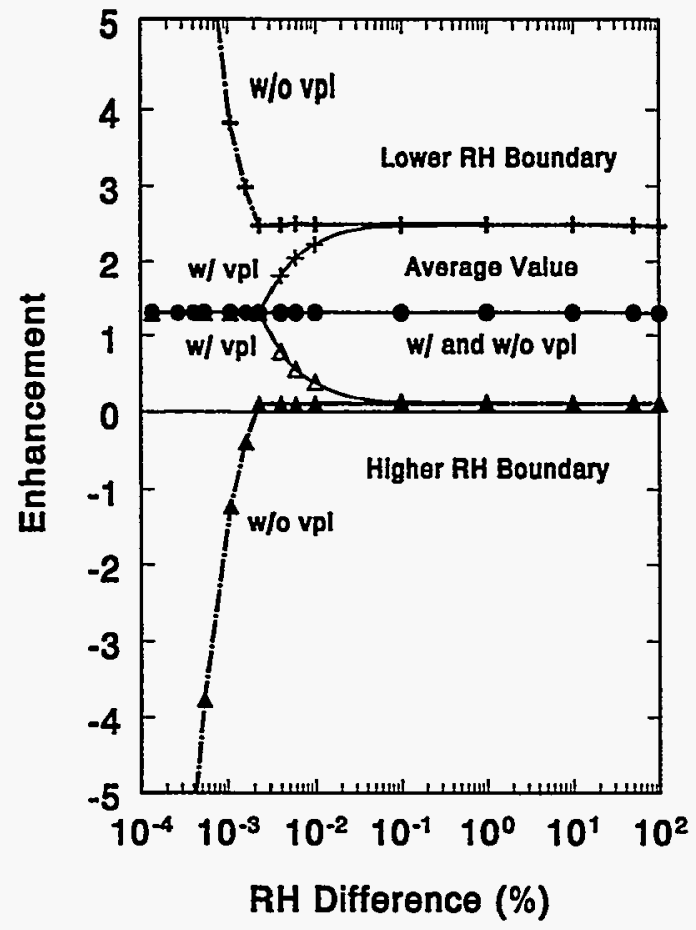

Figure 3-25

Enhancement Factors

with and without VPL

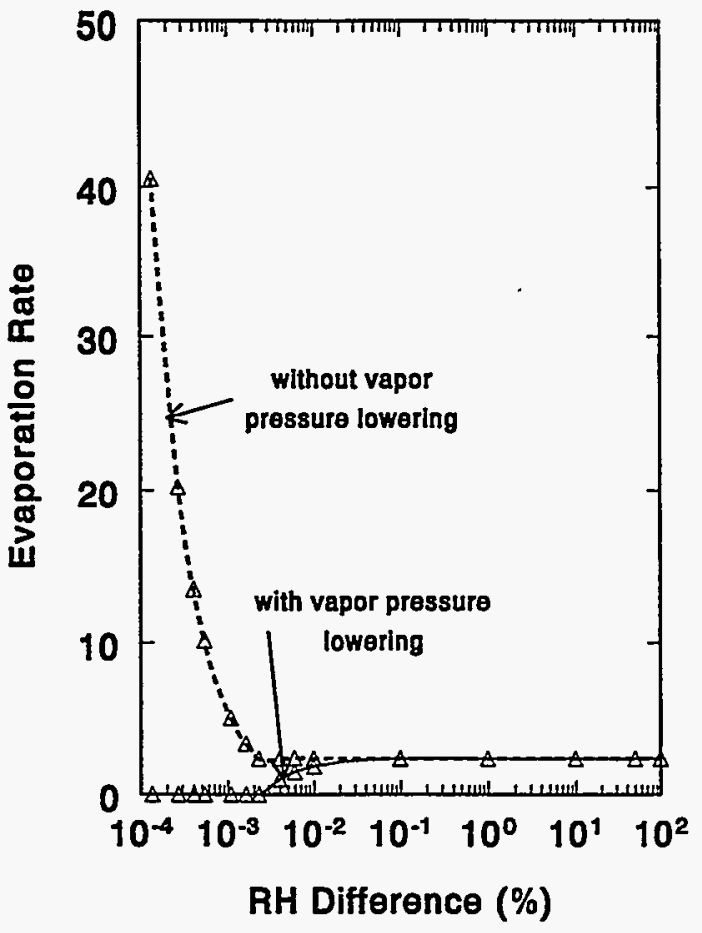

Figure 3-26

Evaporation Rate with and without VPL 
The influence of the boundary conditions on the net evaporation rate from the liquid island is important as shown in Figure 3-26; results with and without vapor pressure lowering are included. For steady-state conditions without vapor pressure lowering, the evaporation rate is large and decreases with increasing RH difference; in contrast, the net evaporation rate with vapor pressure lowering is zero. For transient conditions, the evaporation rate without vapor pressure lowering is constant. The results with and without vapor pressure lowering converge as the $\mathrm{RH}$ difference increases.

\subsection{Discussion}

Enhanced vapor diffusion occurs under steady-state and transient conditions. While the details of the pore-scale behavior are a function of steady-state or transient conditions and the magnitude of the RH difference, the average enhancement factor based on the average mass flux is constant. These results have important implications on experimental and modeling efforts. As discussed in Gu et al. (1998), steady-state vapor diffusion experiments are difficult to perform, and only transient data were obtained. Based on the present study, their enhancement factors using the average vapor mass flux do not need to be corrected for transient effects and have the same numerical value as for steady-state conditions. These results also simplify the modeling effort because evaporation and vapor diffusion mass flow rates can be combined using a single enhancement factor.

In many previous analyses of vapor diffusion, vapor pressure lowering has been ignored. While the magnitude of vapor pressure lowering is numerically small compared to the total vapor pressure, the effect on vapor diffusion on the pore scale can be significant as shown in this study. Vapor pressure lowering can dramatically influence the liquid island evolution as indicated by the net liquid island evaporation rate, especially for small RH differences.

The magnitude of the vapor diffusion enhancement factor is a function of many different parameters, and the value given in this paper should not be considered to be representative of all conditions. For example, Webb and Ho (1997) calculated enhancement factors up to and greater than 10. The magnitude also changes dramatically with the location of the liquid island. For example, in the present model, if the liquid island were moved to the pore next to the lower $\mathrm{RH}$ boundary, the average enhancement factor would jump from 1.30 to 7.65 .

It should be kept in mind that the present results are with a concentration gradient only; there are no temperature or pressure gradients. In addition, the $\mathrm{RH}$ gradient is imposed over three particle diameters, or $330 \mu \mathrm{m}$. It also must be noted that these conclusions are based on modeling results only, and that pore-scale modeling is only "a useful concept rather than a physical reality". Nevertheless, these results indicate that vapor pressure lowering should be considered in future pore-scale modeling efforts. 


\subsection{Conclusions}

The conclusions from the present pore-scale modeling study of transient and steady-state vapor diffusion under partially-saturated conditions under a concentration gradient are:

1. The details of vapor diffusion on the pore scale in the presence of its own liquid are strongly affected by the boundary conditions of the model. The flow patterns and enhancement factors across the boundaries vary with the magnitude of the $\mathrm{RH}$ difference.

2. The enhancement factor is constant with respect to boundary conditions if it is based on the average vapor flux. Therefore, data such as $\mathrm{Gu}$ et al. (1998) do not need to be corrected for transient effects if the enhancement factors are based on the average value.

3. Vapor pressure lowering should be considered in pore-scale analysis of vapor diffusion in the presence of its own liquid. While the magnitude of vapor pressure lowering may be small compared to the total vapor pressure, it can have significant impact on local vapor condensation, evaporation, and the evolution of liquid islands within the pores.

\subsubsection{Temperature Gradient Boundary Conditions}

Temperature gradient boundary conditions have been applied to the above twodimensional pore network model in order to simulate nonisothermal conditions. Steady-state concentration and temperature gradients are imposed across the model for the present simulations; a zero pressure gradient is assumed. The top and bottom are symmetry boundaries.

Due to vapor pressure lowering at the liquid island interface, the equilibrium vapor pressure is slightly lower than saturated vapor. The equilibrium vapor pressure of the liquid island used in this study is reduced by $0.033 \mathrm{~Pa}$ compared to the saturated vapor pressure at $20^{\circ} \mathrm{C}$, or a relative humidity $(\mathrm{RH})$ of 99.9986 percent. The $\mathrm{RH}$ difference across the model was 0.0013 percent, which was centered around the liquid island equilibrium vapor pressure, or an $\mathrm{RH}$ gradient of approximately $0.039 / \mathrm{m}$. A small temperature gradient is also imposed across the model. The temperature difference is $0.0002^{\circ} \mathrm{C}$ centered around $20^{\circ} \mathrm{C}$, or a model temperature gradient of approximately $0.61{ }^{\circ} \mathrm{C} / \mathrm{m}$.

In many situations, such as for saturated vapor conditions, the concentration and temperature gradients are dependent on each other through the saturated vapor pressure, and the two gradients can be related. However, under other conditions, such as in the near surface of the vadose zone or in drying problems, the two parameters may be completely unrelated. For example, at the surface of the vadose zone, the temperature may be higher than in the subsurface, while the vapor concentration at the surface may be lower than in the subsurface, especially for arid conditions. Therefore, the two gradients are considered to be independent of each other in the present paper for generality. This approach is consistent with gas-phase diffusion, where concentration and temperature gradients are considered separately. 


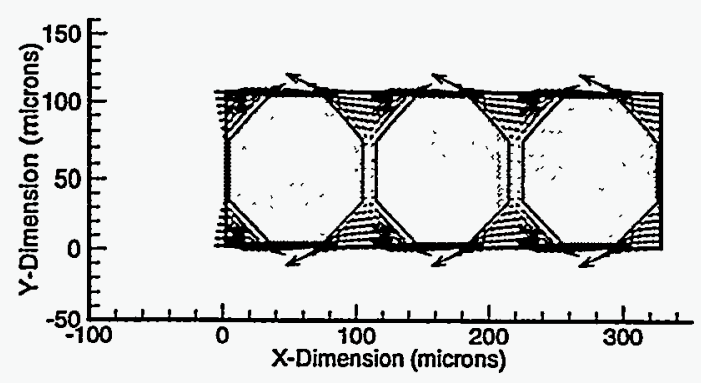

(a) Water Vapor Velocity Vectors

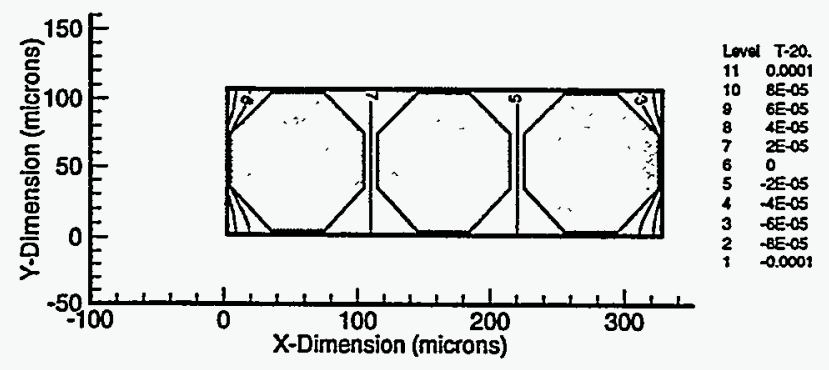

(b) Temperature Contours

Figure 3-27

Results for All-Gas Conditions With Concentration and

Temperature Gradient Boundary Conditions

\subsection{Results}

The basic model used in the present study was shown earlier. The liquid island is located in the top center throat of the model. Figure 3-27 shows the vapor velocity vectors and temperature contours for all-gas conditions with a temperature gradient; vapor velocity results with and without a temperature gradient are essentially identical. This result is not surprising because diffusion in the model is only driven by a concentration gradient; the Soret effect due to a temperature gradient is not included. Vapor diffuses from right to left. The velocity increases significantly in the pore throats of the model. Although not shown, air diffuses from left to right with a slightly larger mass flow rate in agreement with Graham's Laws (see Webb, 1998b). The temperature gradient is symmetrical between the top and the bottom.

Figure 3-28 presents the results with a liquid island with no temperature gradient; these results are the same as given in earlier studies. Vapor essentially diffuses "through" the liquid island due to condensation/evaporation processes. The vapor diffusion rate is about 30 percent higher than for all-gas conditions, or an enhancement factor of about 1.30, as indicated by the longer velocity vectors. The temperature contours due to the liquid island phase change are also given in Figure 3-28. These temperature differences are extremely small and may be about the numerical noise level of the calculation, although the symmetry of the results is encouraging. 


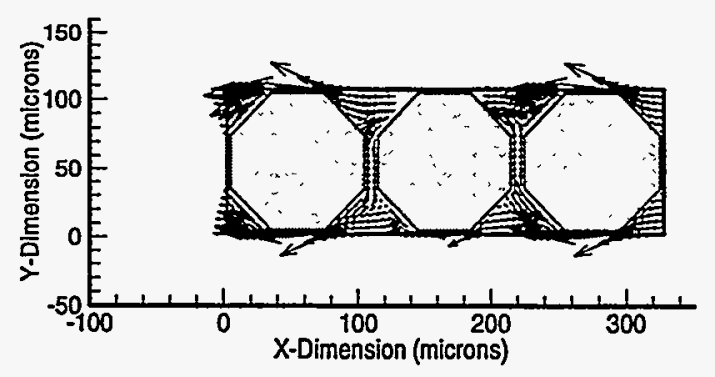

(a) Water Vapor Velocity Vectors

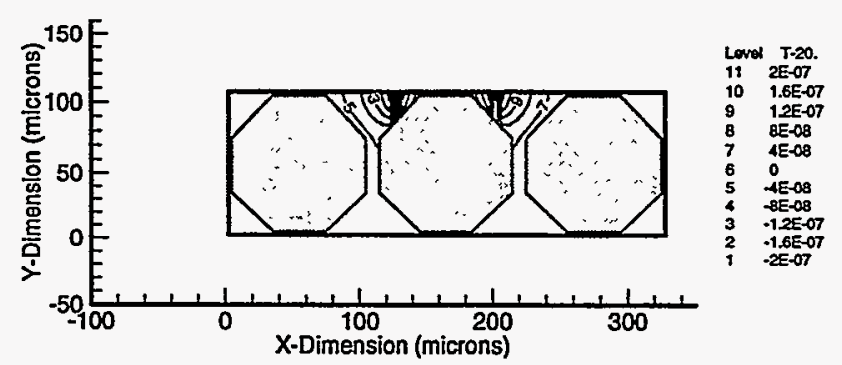

(b) Temperature Contours

Figure 3-28

Results for A Liquid Island With Concentration Boundary Condition

The results in figures 3-29 and 3-30 are for a liquid island including a temperature gradient in addition to the concentration gradient. Figure 3-29 is for an aiding temperature gradient, while Figure 3-30 is for an opposing temperature gradient. The definition of aiding and opposing temperature gradients is based on thermal diffusion for water vapor. As measured by Gu et al. (1998), water vapor diffusion under a temperature gradient goes from hot to cold. Similarly, the model of Philip and deVries (1957) predicts enhanced vapor diffusion from hot to cold. Therefore, an aiding temperature gradient is where both gradients presumably act to promote vapor diffusion. In this case, one boundary would have a higher concentration and higher temperature (HC-HT), while the other boundary would have a lower concentration and lower temperature (LC-LT). For an opposing gradient, the temperature gradient would be reversed, or the boundary conditions would be (HC-LT) and (LC-HT).

The vapor velocity vectors change slightly with the imposition of a temperature gradient, although the general behavior remains the same. An aiding temperature gradient (HC-HT to LCLT) decreases the vapor diffusion rate slightly from 1.30 to 1.19 , while the opposing gradient (HC-LT to LC-HT) increases the enhancement factor from 1.30 to 1.40 . Thus, an aiding temperature gradient actually decreases the vapor diffusion rate, while an opposing temperature gradient increases the vapor diffusion rate. Note that the temperature contours for the two cases overlay one another. 


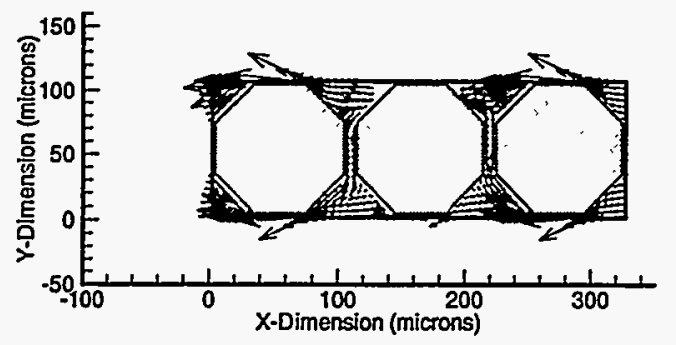

(a) Water Vapor Velocity Vectors

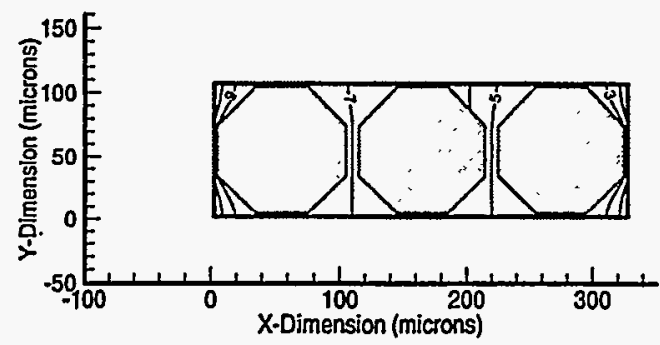

(b) Temperature Contours

Figure 3-29

Aiding Temperature Gradient

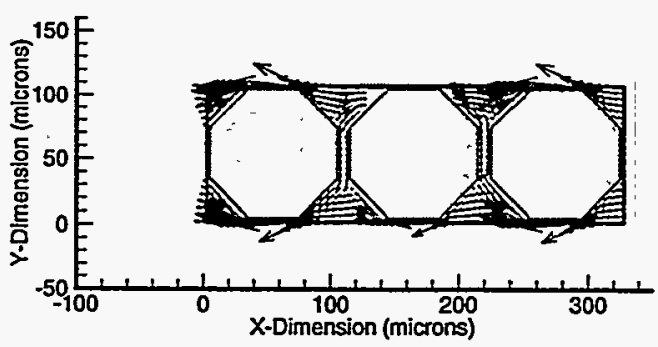

(a) Water Vapor Velocity Vectors

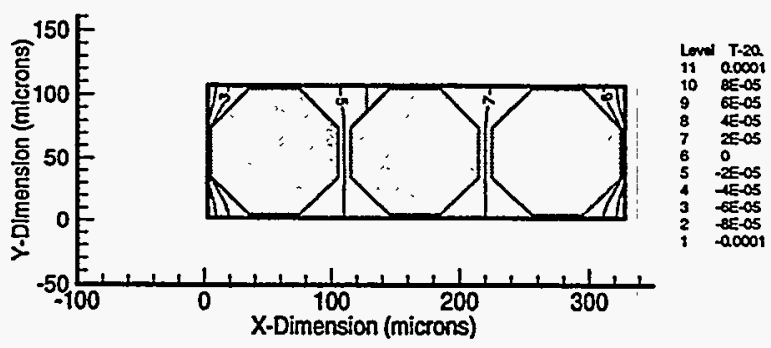

(b) Temperature Contours

Figure 3-30

Opposing Temperature Gradient

\section{Results for Liquid Island With Concentration Gradient Temperature Gradient Boundary Conditions}

The processes responsible for the influence of the temperature gradient need to be examined. Philip and deVries (1957) postulated that there would be an increased temperature gradient in the gas phase which would lead to enhanced vapor diffusion. This same process may be expected to be operative for all-gas conditions, but no enhancement was seen in the present model calculations. The processes responsible for the temperature effect in the present calculations were studied by imposing a temperature gradient without any concentration gradient. Somewhat surprisingly, vapor diffusion occurred with no concentration gradient. The enhancement factor was about 0.106 compared to all-gas conditions with a concentration gradient, or the difference in the enhancement factors seen previously for a temperature gradient.

Figure 3-31 shows the velocity vectors and temperature contours for this case of a temperature gradient with no concentration gradient (left side hot and right side cold). The velocity vectors scale is ten times larger than in the previous vapor velocity figures for clarity. Due to the imposed temperature gradient, vapor evaporates from the warmer end of the liquid island and condenses on the cooler end, setting up an internal recirculation pattern. The higher vapor pressure at the warmer end causes vapor diffusion to the left boundary, while the lower vapor pressure at the cooler end causes vapor to flow into the model from the right boundary. For all-gas conditions, there is no vapor diffusion for these same boundary conditions. 


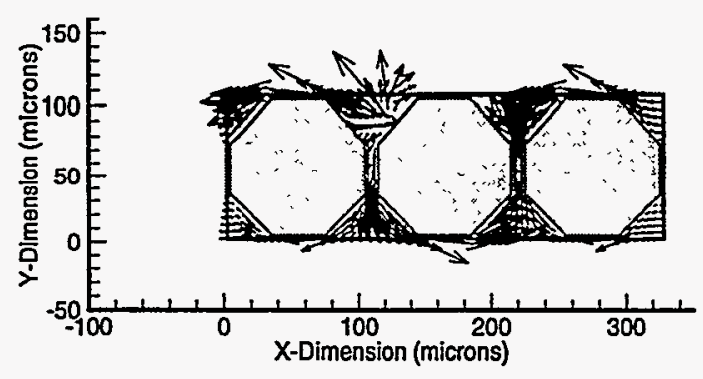

(a) Water Vapor Velocity Vectors - 10x Scale

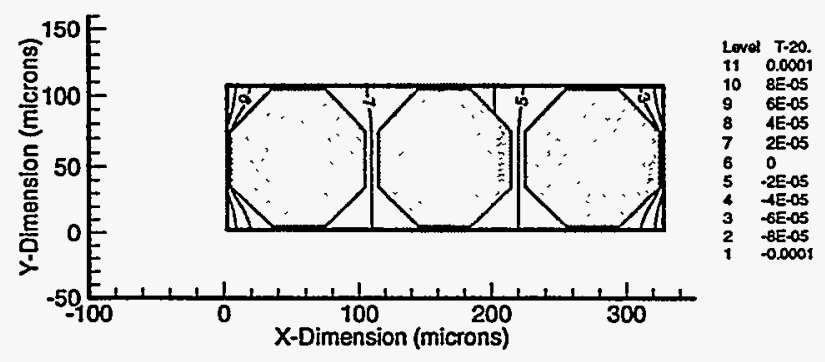

(b) Temperature Contours

Figure 3-31

Results for Liquid Island With No Concentration Gradient and Opposing Temperature Gradient Boundary Conditions

The above results indicate that vapor diffusion due to a temperature gradient causes transport from the cold boundary to the hot boundary. Results from Gu et al. (1998) indicate that thermal diffusion based on free-space thermal diffusion of water vapor in air causes transport from hot to cold. The net effect is that the liquid island processes appear to act opposite the Soret effect. Using the Soret coefficient of $3.9 \times 10^{-9} \mathrm{~kg} / \mathrm{m}-\mathrm{s}$ (Gu et al., 1998) and the imposed temperature gradient, the expected thermal diffusion mass flux is about 34 percent of the concentration gradient mass flux. The high percentage of thermal diffusion is due to the large temperature gradient relative to the concentration gradient in the model. Based on the temperature gradient enhancement of 0.106 , the net effect is about -35 percent $(0.106 / 0.30)$ of the concentration mass flux. Because the effects are opposite (Soret coefficient is hot to cold; liquid island effects are cold to hot), the net effect of a temperature gradient may be negligible.

Finally, the effect of the solid-solid contact area fraction has been studied. All of the above results are for a contact area of 0.2 percent; the contact area has been increased to 2.0 percent for this sensitivity study for an opposing temperature gradient. The vapor velocity vectors (not shown) are slightly greater than in Figure 3-29, although the difference is small. The enhancement factor increases from 1.40 to 1.46, or only about a 4 percent increase for an order of magnitude increase in the solid-solid contact area. The temperature contours (not shown) are influenced much more than the velocity vectors and are more evenly distributed than in Figure 329 due to the higher contact area. The larger difference in the temperature contours, and the 
resulting minimal increase in the vapor diffusion rate, supports the minor effect of the gas phase temperature gradient on enhanced vapor diffusion.

\subsection{Discussion}

Pore-scale modeling of enhanced vapor diffusion under concentration and temperature gradients indicate that the concentration gradient effect is predominant; the temperature gradient effect is of lesser importance. The direction of the temperature gradient (aiding or opposing) has an impact on the enhancement factor. An aiding temperature gradient (based on the Soret coefficient) actually decreases the enhancement factor, while an opposing temperature gradient increases the enhancement factor. The temperature influence from the liquid island produces vapor diffusion from the cold boundary to the hot boundary. This direction is the opposite of the Soret effect, which causes diffusion of water vapor from the hot boundary to the cold boundary.

Due to the possible importance of the temperature gradient, the solid-solid contact area was increased by an order of magnitude. This increase was performed to see if the temperature gradient effect was influenced by the actual pore-scale temperature distribution. Only a minimal change $(\sim 4 \%)$ in the enhancement factor was noted.

Table 3-3 summarizes the enhancement factors calculated in this study. The magnitude of the vapor diffusion enhancement factor is a function of many different parameters, and the values given in this paper should not be considered to be representative of all conditions. For example, Webb and Ho (1997) calculated enhancement factors up to and greater than 10. The magnitude also changes dramatically with the location of the liquid island. For example, in the present model, if the liquid island were moved to the pore next to the lower RH boundary, the average enhancement factor would jump from 1.30 to 7.65 .

The present modeling results provide additional mechanisms beyond those postulated by Philip and deVries (1957). As discussed earlier, their approach assumes that enhanced vapor diffusion is driven by the thermal gradient; without a thermal gradient, no enhancement occurs. In the model results shown above, concentration gradients are the dominant driving force for EVD; temperature gradients are a minor influence. While it is recognized that Philip and deVries (1957) used a concentration gradient in their initial equation for diffusion, their subsequent expansion of the equation (see Nakano and Miyazaki, 1979, for a minor correction) and various assumptions arrived at enhancement of the thermal component only. Therefore, their model may need to be revisited based on these modeling results. 
Table 3-3

Summary of Enhancement Factors

\begin{tabular}{|c|c|}
\hline Conditions & $\underline{\text { Enhancement }}$ \\
\hline Factor \\
\hline All-Gas With Concentration Gradient \\
- No Temperature Gradient & 1.00 \\
- With Temperature Gradient & 1.00 \\
\hline Liquid Island With Concentration Gradient & \\
- No Temperature Gradient & 1.30 \\
- Aiding Temperature Gradient & 1.19 \\
- Opposing Temperature Gradient & 1.40 \\
10x Contact Area & 1.46 \\
\hline Liquid Island Without Concentration Gradient & 0.106 \\
\hline
\end{tabular}

\subsection{Conclusions}

The present pore-scale modeling studies using concentration and temperature gradients have resulted in the following conclusions:

1. For enhanced vapor diffusion, the concentration gradient effect is predominant. The temperature gradient effect is of lesser importance.

2. The direction of the temperature gradient (aiding or opposing) has an impact on the enhancement factor. An aiding temperature gradient decreases the enhancement factor, while an opposing temperature gradient increases the enhancement factor. The direction of the liquid island enhancement is opposite of the Soret effect.

3. The present modeling results provide additional mechanisms for EVD beyond those considered by the model of Philip and deVries (1957). Their model indicated that enhancement would only occur due to temperature gradients. The model may need to be modified to reflect the present results.

4. The effect of increasing the solid-solid contact area on the enhancement factors is minimal. 


\subsection{Discussion}

Analytical and numerical pore-scale analyses have been performed. The analytical analyses considered the various processes in a more qualitative manner and calculated significant enhancement of vapor diffusion. These results indicated that development of a numerical pore-scale model was warranted. The TOUGH 2 code was used to develop this pore-scale model, which is the first mechanistic model of enhanced vapor diffusion. The mechanistic model calculated significant enhanced vapor diffusion in steady-state and transient conditions. All the above approaches reached the same conclusion - enhanced vapor diffusion (EVD) is possible and may be significant. Vapor diffusion in the presence of liquid islands behaves considerably different than gas diffusion and should be treated differently.

The approaches considered in this section also differ considerably from the model of Philip and deVries concerning the driving force for EVD. While the analytical pore-scale approach with a temperature gradient reached similar conclusions to Philip and deVries, the concentration gradient pore-scale analysis and the mechanistic models reached different conclusions. According to these studies, EVD can be driven by a concentration gradient without the need for a temperature gradient. This result provides additional mechanisms beyond the model of Philip and deVries who assumed that a temperature gradient was necessary for EVD to occur.

In fairness, a concentration gradient and temperature gradient are often intertwined, such as the variation of the saturated vapor pressure with temperature. However, there are other situations such as drying where the vapor pressure is significantly less than saturation, so this separation of concentration and temperature gradients is necessary. In any event, the model of Philip and deVries should be revisited based on the present results, which include the experiments summarized in the next section.

While the present model indicates that enhanced vapor diffusion is possible, only experimental data can confirm the process. As noted by Ewing and Gupta (1993), pore-scale modeling is a "useful concept rather than a physical reality". The present authors support this notion. Experimental data obtained as part of this LDRD are summarized in the following section. 


\subsection{Summary of Experimental Data}

\subsection{Introduction}

As part of this LDRD, experiments that measured vapor diffusion directly were conducted to confirm the existence of EVD. The approach evaluates EVD at three different length scales; the pore scale, a two-dimensional network as represented by a fracture aperture, and a porous medium. These measurements are the first known experiments to obtain direct data for enhanced vapor diffusion.

Pore-scale experiments have been conducted at New Mexico Tech to investigate EVD at an individual pore scale where the mechanisms involved are well controlled. The experiments are made from glass to permit direct observation of the processes involved. These tests are complete, and the data are reduced. Some of the data will be presented in this summary, and the final report is expected to be available shortly.

At Sandia, a fracture aperture setup consisting of two roughened glass plates is being used to look at EVD in two dimensions including the effects of isolated liquid zones. As with the pore-scale tests, the plates are made of glass to permit direct observation of the processes. The pore-scale and fracture aperture experiments include visualization so the processes involved can be directly observed. Preliminary experiments have been completed, and final test apparatus modifications are underway. The experiments are not quite finished at present.

Finally, porous media tests were conducted at Washington State University using a packed bed of glass beads. These experiments are of a more-general three-dimensional geometry, which limited visualization. These tests have recently been completed. Two conference papers on the experimental data and modeling efforts have been written (Gu, 1998, 1999). These experiments benefitted from the fact that an existing experimental apparatus was used in the present experiments, which only needed minor modifications for the present study.

All three experimental investigations are discussed in more detail below, and the data obtained to date are summarized. The details for each experiment are different due to their varying states of completion.

\subsection{Pore-Scale Experiments (New Mexico Tech)}

The main purpose of the experiments conducted at New Mexico Tech was the direct measurement of EVD in a pore-scale geometry. These tests were conducted by Thomas Silverman, a graduate student, under the direction of Professor John L. Wilson of the Hydrology Program in the Earth and Environmental Science Department.

As discussed earlier, EVD is driven by condensation/evaporation across isolated liquid islands. The purpose of the New Mexico Tech tests was to investigate this mechanism in an 
individual pore. Controlling the temperature gradient in a pore-scale experiment is difficult. Therefore, it was decided to measure EVD under a concentration gradient. As shown by Webb and Ho (1997), significant enhanced vapor diffusion should also exist under these conditions.

In order to achieve reasonable vapor diffusion rates in a single pore, significant vapor pressure lowering must occur. As calculated by Webb and Ho (1997), a pore throat of $5 \mu \mathrm{m}$ results in only a $0.5 \mathrm{~Pa}$ reduction in the vapor pressure compared to a saturated vapor pressure of $2337 \mathrm{~Pa}$ at $20^{\circ} \mathrm{C}$. In order to obtain significant vapor pressure lowering without resorting to extraordinarily small dimensions, it was decided to induce vapor pressure lowering through the use of water-brine mixtures. On one side of the pore, essentially saturated water vapor conditions exist, while on the other side of the pore, a reduced vapor pressure is imposed through the use of a $\mathrm{LiCl}$ brine solution. The liquid island in the middle has a diluted brine solution with a vapor pressure approximately midway between the end states. This configuration is stable because if the liquid island has too high of a brine concentration, condensation from the pure water vapor source will be greater than evaporation to the brine source, resulting in dilution of the liquid island concentration. Conversely, if the liquid island concentration is too low, net evaporation will occur, and the brine concentration in the liquid island will increase.

The most significant problem is designing the test was the elimination of leakage of vapor from the test section. Numerous designs and sealing materials were investigated until the final setup was developed. The test section consists of two vertical columns with a pore of glass "drawn" between the two columns as shown in Figure 4-1. The minimum pore dimension is approximately $1 \mathrm{~mm}$. The column on the left has essentially pure water, while the column on the right has brine with a solid of the salt material $(\mathrm{LiCl})$ in order to maintain saturated brine conditions. There are openings at the top of each column to introduce the various liquids and the liquid island; the openings are sealed with rubber septa. The tips of two hypodermic syringe needles can be seen sticking through the septa in Figure 4-1b.

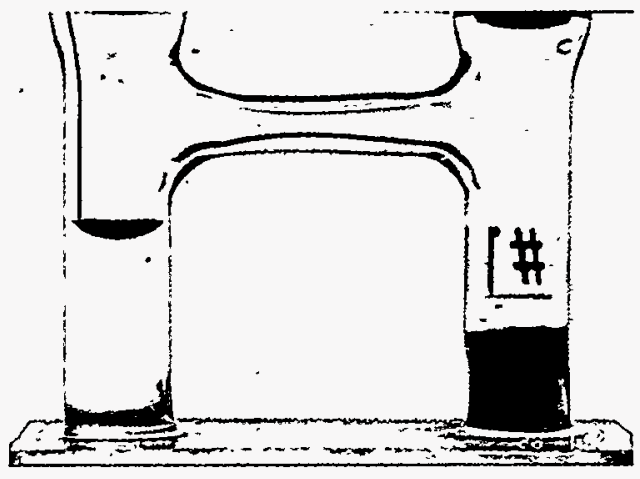

(a) All-Gas Conditions

Figure 4-1

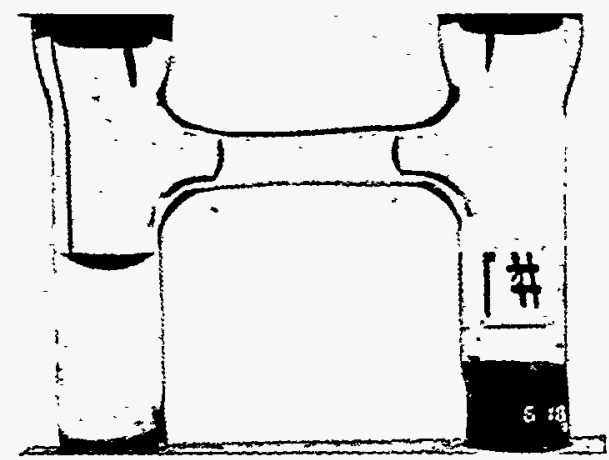

(b) Liquid Island Conditions

Pore-Scale Experimental Apparatus - Single Pore 
The above single-pore design has a drawback in that the vapor is essentially "forced" to diffuse through the liquid island. Therefore, a test design with two parallel pores was made as shown in Figure 4-2.

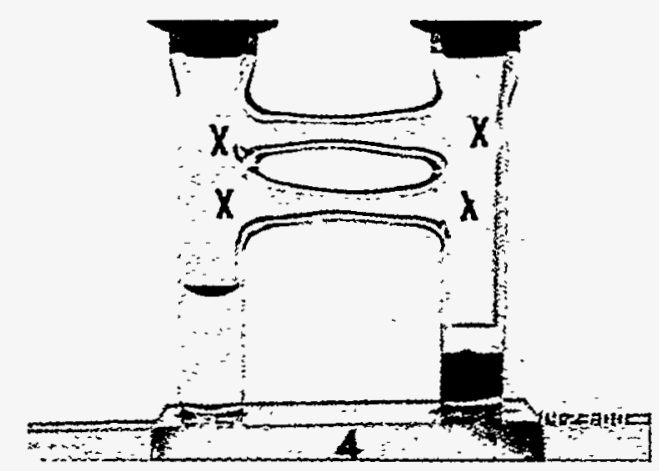

Figure 4-2

Pore-Scale Experimental Apparatus - Two Pores

Tests were conducted using a concentration difference imposed by the variation in the vapor pressure of the liquid brines. No temperature gradient was imposed. Note that the model of Philip and deVries (1957) assumes that EVD will only exist if there is a temperature gradient; therefore, the New Mexico Tech experiments should not see EVD. In contrast, the pore-scale modeling of Webb and Ho (1997) showed significant enhancement with a concentration gradient without a temperature gradient. These tests confirm the results of Webb and Ho (1997).

Some experimental data for the single-pore model are given in Figure 4-3. The drawdown rate in the pure-water column is plotted as a function of time for seven different liquid island lengths, including zero. As can be seen, the drawdown rate, and in effect the vapor diffusion rate, is extremely constant with time and increases significantly with liquid island length. The drawdown rates can be fit with a straight line giving a measure of the relative diffusion rate of the cases. This relative rate, which can be viewed as the enhancement relative to no liquid island, is plotted in Figure 4-4. For no liquid island, there is no enhancement, so the factor is 1.0. For the longest liquid island, the enhancement factor is about 3.

Additional tests were also performed with the two-pore model including gas/gas, gas/liquid, liquid/gas, and liquid/liquid tests (the fluid states $I / j$ refer to the top and bottom pores, respectively). These data will be included in the final report.

These experimental results confirm the existence of EVD on the pore scale. In addition, they demonstrate that EVD can occur due to a concentration gradient; a temperature gradient is not necessary. These results confirm the results of Webb and Ho (1997) and provide additional mechanisms for EVD beyond those given by the model of Philip and deVries (1957). 


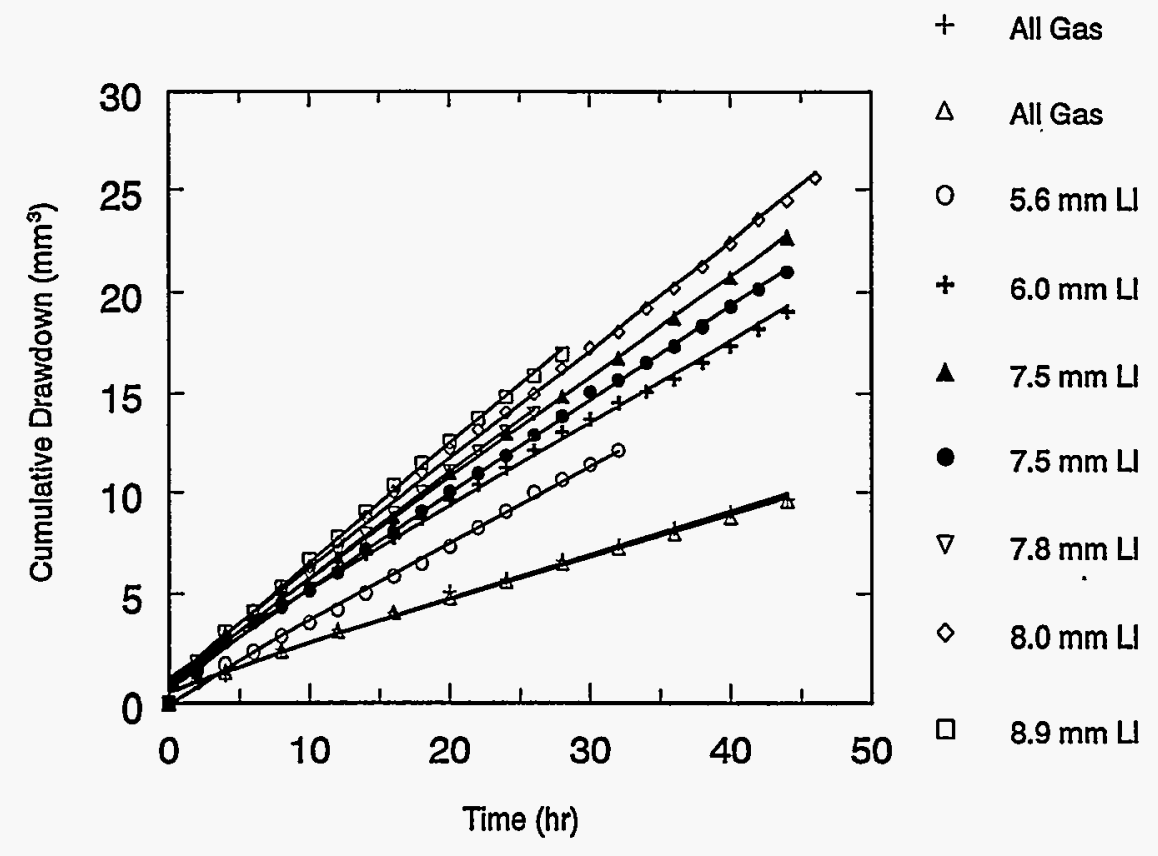

Figure 4-3

Single Pore Pure Water Drawdown Rate

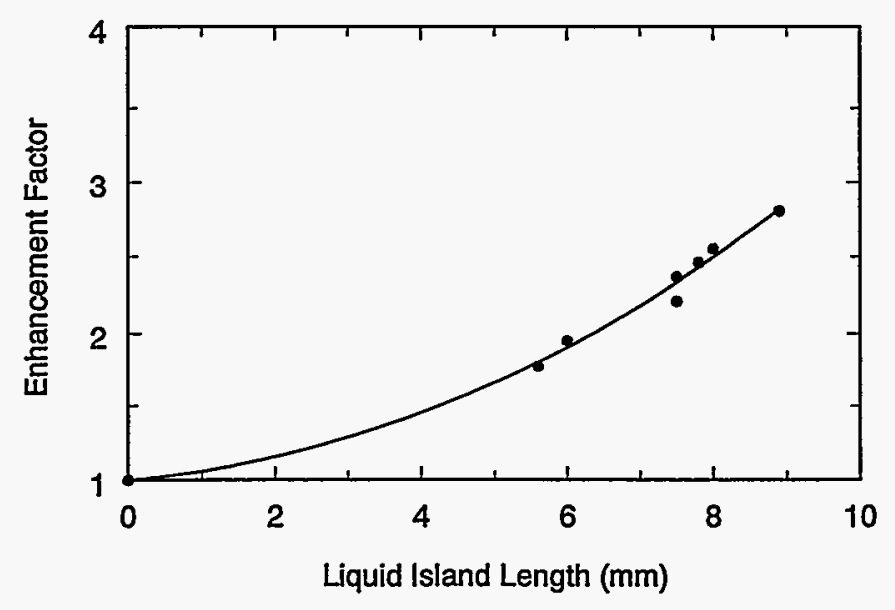

Figure 4-4

Enhancement Factors Vs. Liquid Island Length 


\subsection{Two Dimensional Network - Fracture Aperture Experiments (SNL)}

Fracture aperture experiments are being conducted in the Flow Visualization and Processes Laboratory (FVPL) at Sandia. These experiments are being conducted by Donald Fox under the direction of Robert J. Glass. The objective of these experiments is to measure enhanced vapor diffusion in a two-dimensional geometry where phase structure can be visualized in a geometrically complex system. The pore-scale tests at New Mexico Tech were focused on evaluation of mechanisms on a pore-scale with well-controlled conditions including geometry and visualization. The porous media tests at Washington State University are for a threedimensional geometry with limited visualization. The fracture aperture tests combine the two experiments by testing a complex geometry in two-dimensions with good visualization. For this purpose, a single fracture system using roughened glass plates has been used. This experimental system (see figure 4-5) has been used at the FVPL for other process elucidation studies including gravity driven fingering (Nicholl et al., 1994) and phase dissolution (Glass and Nicholl, 1995).
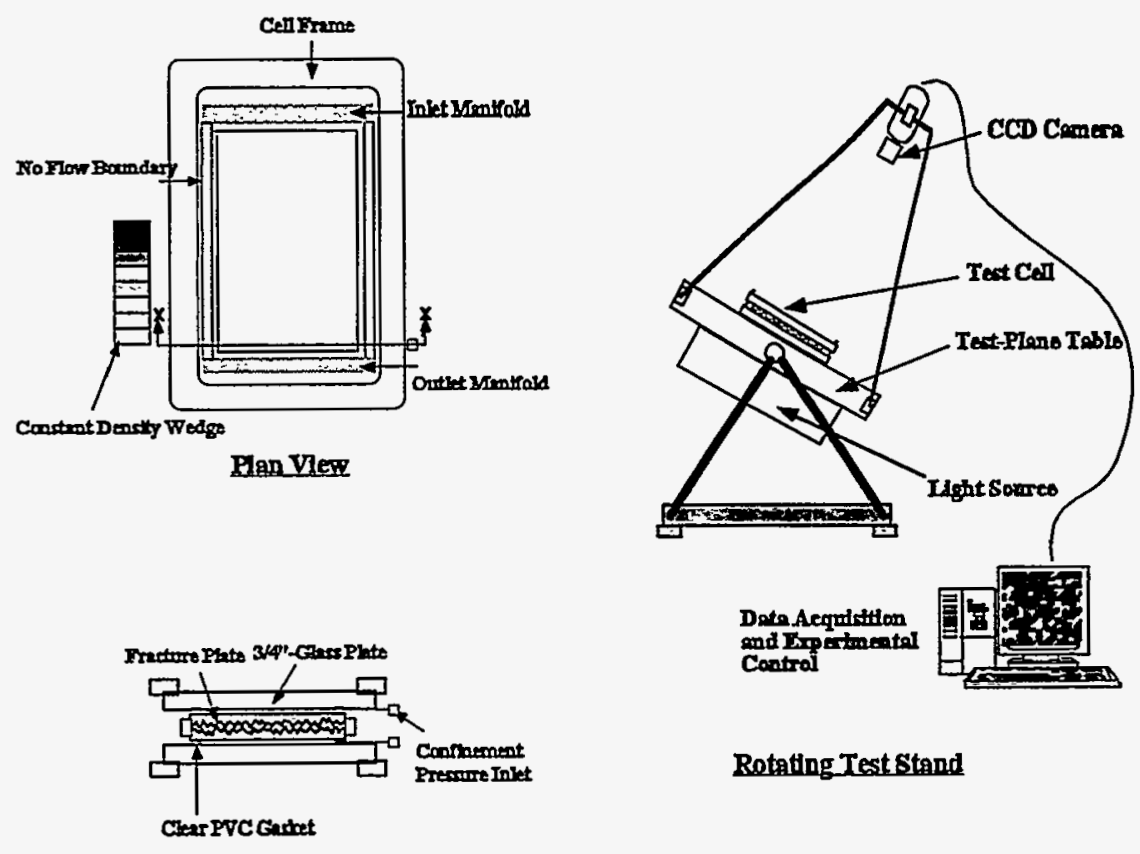

Rotafing Test Stand

Cross-Sectlon X-X'

Figure 4-5

Light Transmission Experimental System

The conceptual design for the experiment is a constant temperature, single fracture system subjected to transient evaporation between an upstream gas reservoir connected to liquid and a downstream reservoir at very low, controlled vapor pressure. Three initial conditions are being considered: full liquid saturation, full gas saturation, and continuous gas with fully entrapped liquid islands. The vapor flux through the system including change in liquid mass within the fracture system will be measured with respect to time. The inlet and outlet fluxes will be calculated by measuring the change in mass of upstream and downstream reservoirs. In 
addition, the mass retained within the fracture will be measured using the transmitted light imaging capabilities at the FVPL.

The aperture field of the glass-plate fracture is characterized using a light transmission technique developed and evaluated at the FVPL in the last two years. The technique yields the entire aperture field at the time of the experiment with a spatial resolution within the plane of the fracture of $\sim 0.15 \mathrm{~mm}$ and a total field size of $\sim 25 \times 15 \mathrm{~cm}$. The error in the measurement of local aperture on the glass plate fracture chosen for the EVD experiments has been determined to be $\sim 0.001 \mathrm{~mm}$ with a mean aperture of $\sim 0.1 \mathrm{~mm}$. The gas/liquid occupancy within the fracture is measured using digital images to define the initial condition (See figure 4-6) and as a function of time as the experiment progresses. The liquid volume within the fracture cell at any time is simply the occupancy field times the aperture field, which yields liquid volume within cell.

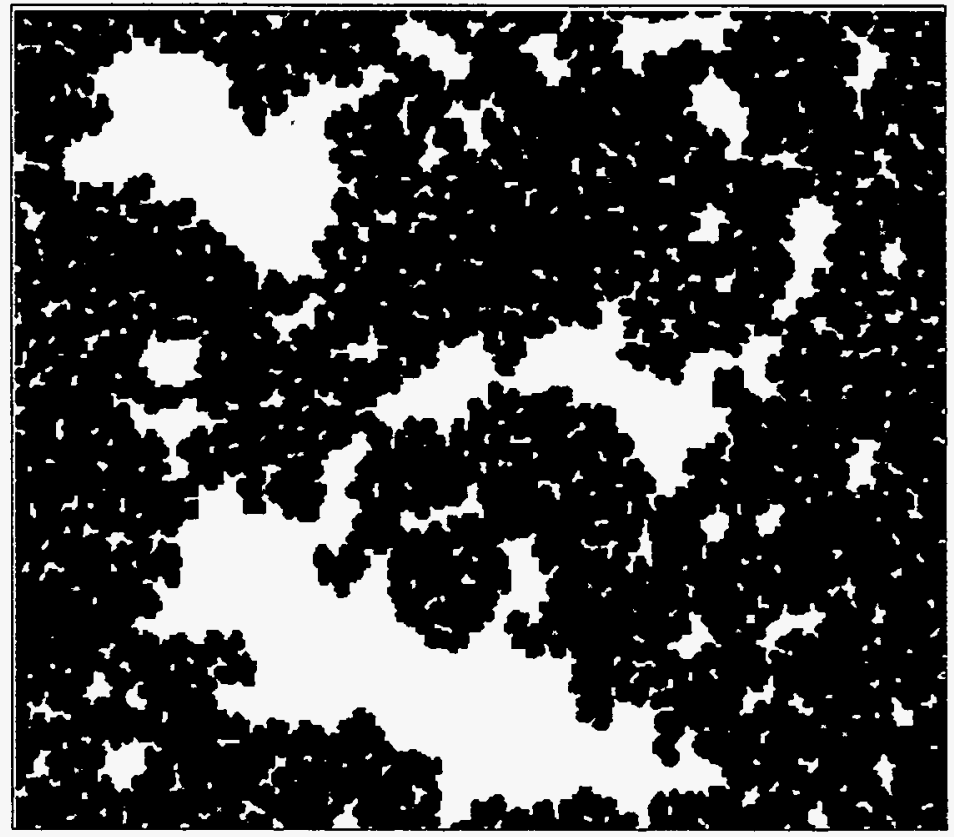

Figure 4-6

Typical liquid island structure at the start of an experiment (white is liquid island, black is gas/vapor)

The inflow reservoir is constructed out of clear PVC with cross-sectional area $\sim 30$ times fracture cross-section. The liquid within reservoir is at atmospheric pressure and is connected to fracture via stagnant gas. The flux out of liquid reservoir is measured by recording the liquid level in time with a digital camera to a resolution of $\sim 0.001 \mathrm{ml}$. The outflow reservoir contains a desiccant and is constructed out of clear PVC containing silica gel beads with active area' 10's to 100 's times greater than the fracture cross-sectional area. The reservoir is removed and weighed in time to measure total vapor flux through system. The test cell is designed for use with higher vapor pressure liquid (alcohol) so that tests can be performed rapidly. Significant effort was invested in the cell design so that it would seal to alcohol vapor. 
Preliminary tests with alcohol were conducted in early June 1998. The purpose of the test was to evaluate cell integrity (leaks to alcohol); consider whether standard photography could be used to measure gas/liquid occupancy; implement and evaluate inflow reservoir volume measurement; implement and evaluate experimental control program; and to determine time scales for experiment. The cell was demonstrated to be leak free over a test period of 21 days while the outflow of the fracture was sealed. After this initial leak test, the downstream fracture edge was opened to the room to begin evaporation. The gas/liquid phase occupancy data from standard photography was found to be much harder to reduce and of lower quality than the high resolution digital camera procedure. The inflow manifold level measured in time using digital camera worked well (see figure 4-7). However, an initial period where volume actually increased within the reservoir was noted and could have been due to the way we began the experiment. Some experimental control program problems were identified and fixed. The time scale for full dryout of the fracture was greater than 35 days when experiment was terminated.

Gas Diffusion Experimental System Setup

Meniscus Position, From Top of COHU Image.

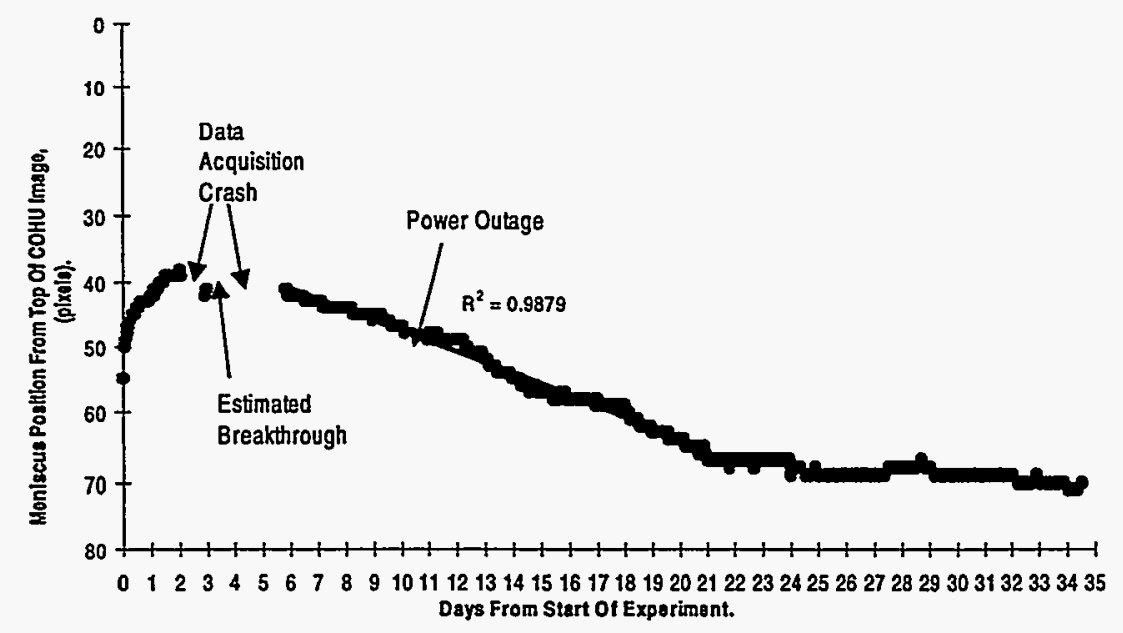

Figure 4-7

Inflow manifold level as a function of time

The final tests are in progress. Silica gel beads are being used as the desiccant in the downstream reservoir. Dye has been added to the alcohol so that we can obtain high quality raw phase structure photographically. A full suite of tests where the initial liquid island geometry is varied has begun. Upon completion, one to three experiments will be reproduced on the light visualization system using the high resolution digital cameras. 


\subsection{Porous Media Experiments (Washington State University)}

The porous media tests were performed by Limin $\mathrm{Gu}$, a graduate student, under the direction of Professor O.A. Plumb of the Department of Mechanical and Materials Engineering at Washington State University. Additional details are given in Gu (1998) and Gu et al. (1998, 1999). The objective of the Washington State University experiments was to obtain vapor diffusion in a three-dimensional geometry. Three types of experiments were conducted in a packed bed of glass beads.

1. Isothermal Experiments. Several isothermal experiments were conducted in free space (no porous media) in order to test the experimental apparatus and technique. A dry-bed tortuosity coefficient was then determined from the observed vapor flux through a dry packed bed under a relative humidity gradient and isothermal conditions.

2. Nonisothermal Experiments. Vapor diffusion under combined thermal and concentration gradients was measured and compared to the dry-bed isothermal experiments to determine the presence of enhanced vapor diffusion. The boundary conditions were essentially constant so the experiment was steady-state or a slow transient.

3. Drying Experiments. Drying experiments were conducted to investigate transient drying conditions.

The experimental apparatus is illustrated in Figure 4-8. The apparatus consists of a 51 $\mathrm{mm}$ wide $\times 51 \mathrm{~mm}$ deep $\times 95 \mathrm{~mm}$ high chamber constructed from acrylic. A bed of glass beads within the chamber serves as the porous medium. The chamber is capped with a jacket through which water can be circulated at a fixed temperature. Immediately below the water jacket is a fibrous wick, which is connected to an external reservoir. The wick provides a supply of saturated water at a fixed temperature. An electronic balance is utilized to monitor the water loss from the reservoir. The top is clamped to the chamber for easy disassembly.

The bottom of the chamber consists of a desiccant tray and a water jacket. The tray can be removed for weighing. The central section of the chamber, which contains the bed, is slip fit into the lower desiccant chamber. A wire screen at the bottom of the chamber supports the glass beads. Five thermocouples measure the temperature at the wick, the top of the bed, the middle of the bed, the bottom of the bed, and the top of the desiccant. The entire apparatus is insulated with $2.54 \mathrm{~cm}$ of foam insulation. The water jackets at the top and bottom of the apparatus are supplied with water from constant temperature baths. A gamma attenuation system is available to measure the saturation profile in the packed bed during the experiment.

For isothermal experiments the entire apparatus was placed in an environmental chamber maintained at the desired temperature. Dry glass beads $(450 \mu \mathrm{m})$ were carefully packed in the acrylic container a small amount at a time while shaking the container. A very consistent average porosity between 0.37 and 0.374 was achieved using this technique. The porosity for each experiment was determined using measurements of bed volume and weight. At the bottom, 


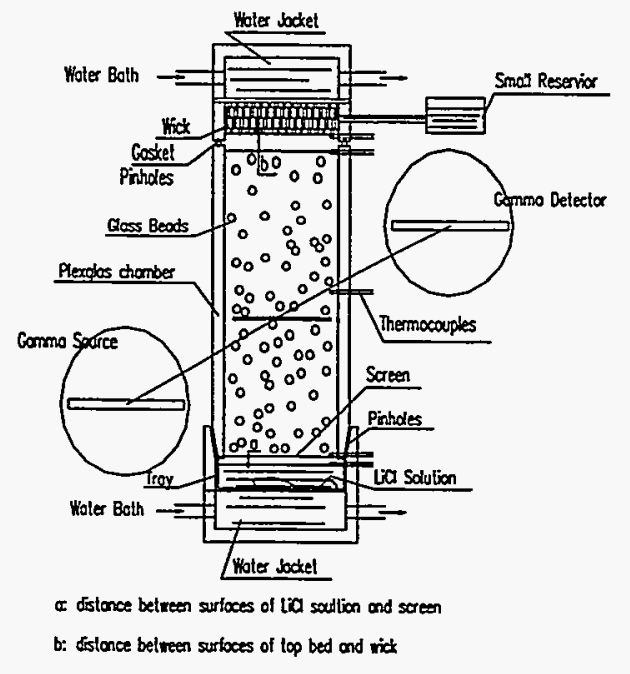

Figure 4-8

Experimental Apparatus

a saturated $\mathrm{LiCl}$ solution was utilized as the desiccant to provide a constant relative humidity of 0.13 , while at the top the saturated wick maintains a relative humidity of unity. Because of the small amount of mass required the wick does not need a continuous supply of water from the attached reservoir for the isothermal experiments. The vapor diffusion rate can be determined by weighing the wick to determine the mass loss and by weighing the desiccant to determine the mass gain. In addition the bed can be weighed to detect any liquid build up in the bed and check the overall mass balance. The electronic balance used for these measurements has an accuracy of \pm 0.01 grams. Weights were recorded every 12 or 24 hours depending on the mass transfer rates. The atmospheric pressure was also recorded twice daily.

For nonisothermal experiments the water circulated through the upper water jacket was maintained at a fixed temperature greater than that circulated through the lower water jacket. The configuration of the temperature gradient (hot on top) resulted in a stable density distribution to eliminate natural convection. Two constant temperature baths were used to supply the water to the jackets. Since the nonisothermal experiments were run for several days, the upper reservoir was utilized to maintain a water supply to the wick. A gamma attenuation system was used to measure the liquid saturation profile in the bed. During nonisothermal experiments water vapor diffusing from the top boundary continuously condensed in the cooler regions of the bed. The mass flux of vapor into and out of the packed bed was determined by weighing the desiccant and the bed.

For the drying experiment, the wick at the top was removed and the tray at the bottom was filled with distilled water instead of the $\mathrm{LiCl}$ solution. The bed was initially saturated to a low level to prevent significant flow in the liquid phase. The top boundary was maintained at a 
higher temperature with no water vapor flux, while the lower water jacket was maintained at a lower temperature and the lower air gap was assumed to be at the saturated vapor condition. During the drying experiments liquid water at the upper warm region of the bed evaporated and diffused to the cold lower region, finally condensing in the water tray. A drying front propagated from the top of the bed to the bottom of the bed. The temperature measurements, saturation distribution measurements and weight measurements were basically the same as those for the nonisothermal experiments.

Before presenting results for experiments involving enhanced vapor diffusion an enhancement factor must be defined. As discussed earlier, there are two different definitions for the enhancement parameter; one normalized using the effective diffusion coefficient for a dry porous medium, which will be termed, $\eta$ and one normalized using the free space diffusion coefficient, which is called $\beta$. Using the measured mass flux, the concentration and thermal diffusion coefficients, and other directly measured parameters, the enhancement factors $\eta$ and $\beta$ can be determined.

The values of these enhancement factors have different ranges and meanings. The factor $\eta$ is a direct measure of the degree of enhancement of vapor diffusion compared to gas diffusion. If $\eta$ is equal to 1.0 , there is no enhancement. In other words, this value indicates that vapor diffusion can be modeled similar to gas diffusion. If $\eta$ is not equal to 1.0 , the applicability of gas diffusion methods to vapor diffusion is questionable. The factor $\beta$ is normalized to free space diffusion values. If $\beta$ is equal to the product of the tortuosity, porosity, and gas saturation, (approximately 0.29), there is no enhancement. If $\beta$ equals 1.0 , vapor diffusion is significantly enhanced and is equal to the diffusion value in free space.

Because of the continuous condensation of vapor in the bed during the nonisothermal experiments, the measurements of the enhancement factor were made under transient conditions. It was possible to establish quasi-steady conditions (for periods of 6 - 10 hours) by adjusting the boundary conditions (temperature) during the course of the experiment. For transient experiments it is noted that the enhancement factor could be based on three different mass fluxes - all of which were measured - the flux entering the bed, the flux exiting the bed, and the average flux. According to modeling results of Webb (1998a), the average flux should be used for the enhancement factors. For the quasi-steady experiments the entering and exiting mass fluxes were equal and hence this problem did not exist.

Figures 4-9 and 4-10 show experimental results for the two enhancement factors (based on the average flux) defined above as a function of average saturation for a number of quasisteady cases and two transient experiments. The transient experiments were conducted using two different temperature differences. For the results identified as transient 1 , the upper and lower bed temperatures were maintained at 39.5 and $27.1^{\circ} \mathrm{C}$, respectively, while for transient 2 , they were maintained at 35.3 and $32.5^{\circ} \mathrm{C}$, respectively. Transient 1 spanned a period of 120 hours beginning with an initially dry bed and ending with an average saturation of 0.19 . Transient 2 ran for 243 hours and ended with a saturation of 0.22 . For the transient experiments 


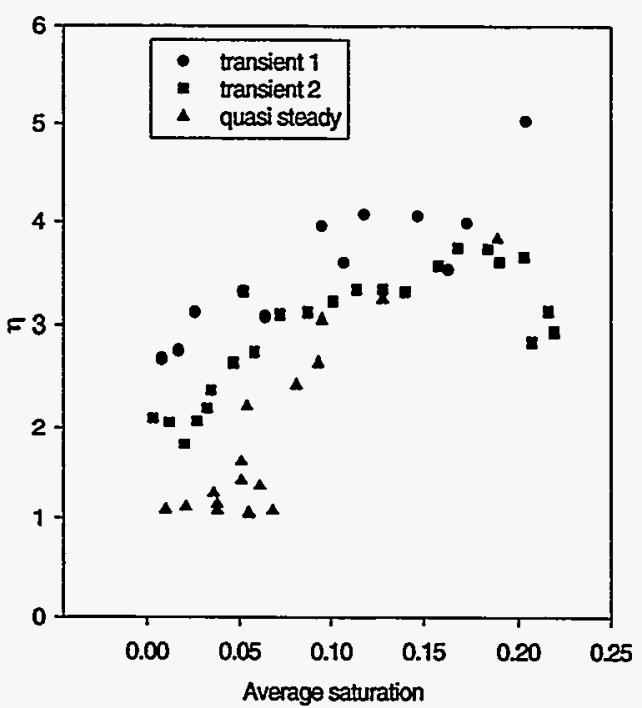

Figure 4-9

Experimental Enhancement Factor, $\eta$

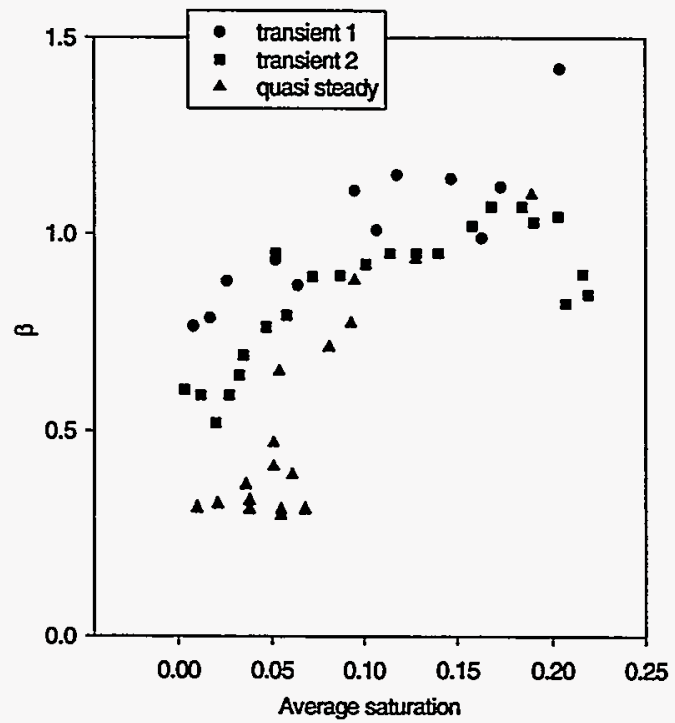

Figure 4-10

Experimental Enhancement Factor, $\beta$ 
enhancement factors are presented based on the average mass flux. The uncertainty in the enhancement factors presented is estimated to be $8.5 \%$.

As can be seen in the figures the results for the quasi-steady measurements fall slightly below those for the transient experiments based on the average of inlet and outlet mass fluxes. The results are qualitatively similar to those of previous investigators (Cass et al., 1984) in that for very low liquid content (saturation less than 0.05 ) there is very little enhancement. As the saturation is increased to 0.20 , where transport in the liquid phase should be expected to begin to contribute, the enhancement factor increases to approximately 1 when compared to free space $(\beta)$ and approximately 3.5 when normalized to the diffusion coefficient for a dry porous medium $(\eta)$. The temperature difference, or temperature gradient, does not appear to have a strong effect on the results. The enhancement factors for the two transient cases which have different imposed temperature gradients are comparable.

Because of the nature of these experiments the saturation distribution in the bed is both nonuniform and time dependent. The saturation distributions as a function of time and space for the two transient experiments are shown in Figures 4-11 and 4-12. In these figures, $x / L=0$ is at the bottom of the bed. The uncertainty in the measurement of saturation is estimated to be $7.5 \%$ when saturation is 0.1 , and $4.3 \%$ when saturation is 0.2 . The primary contributor to this

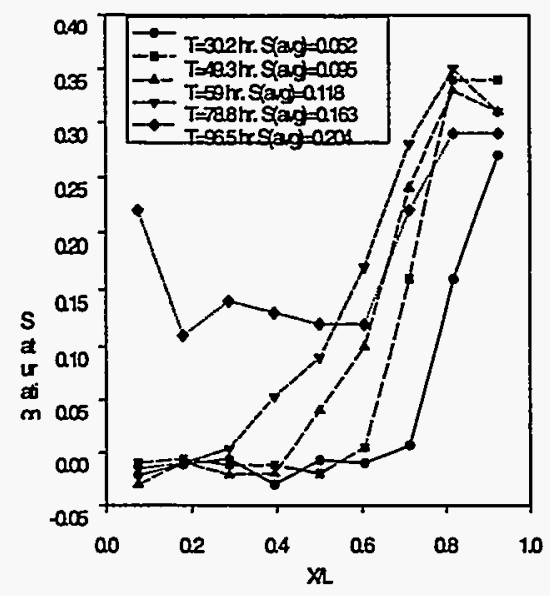

Figure 4-11

Saturation Profile vs. Time, Transient 1 Experiment 


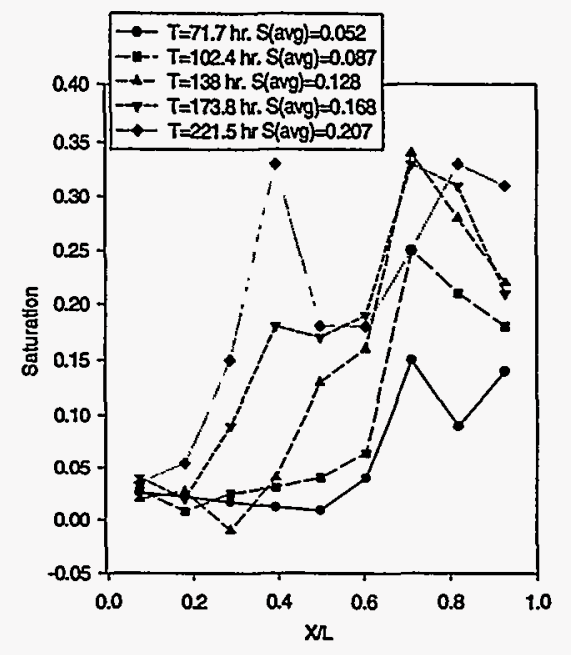

Figure 4-12

Saturation Profile vs. Time, Transient 2 Experiment

uncertainty is the variation in measured gamma counts. In both cases the saturation is high near the top of the bed due to condensation. This region of high saturation increases in thickness as the experiment progresses in time. The wet region is separated from the rest of the bed by a condensation front, which moves toward the bottom of the bed as time progresses. It appears that liquid phase transport begins to play a dominant role as the saturation approaches 0.3 since the saturation does not increase beyond this point. In both experiments the condensation rate exceeds the drying rate and hence the condensation front eventually moves to the lower surface along with higher saturations.

The temperature distributions in the bed are shown in Figure 4-13 for the two transient experiments. As can be seen in the figure, the temperature distributions are not linear and change very little with time in contrast to the saturation distributions. Latent heat exchange and variations in thermal conductivity due to saturation variations both contribute to the non-linear temperature distribution. In the case of a small temperature difference the temperature in the middle of the bed $(x / L=0.5)$ is actually lower than the temperature at the bottom of the bed $(x / L$ $=0.0$ ) due to the dominance of the latent heat associated with evaporation.

The results of one drying experiment are presented. Figure 4-14 shows the saturation profiles in an initially partially saturated packed bed as a function of time. This drying experiment was initialized from a hydrostatic saturation distribution with a temperature of $62.0^{\circ} \mathrm{C}$ at the top of the bed and a temperature of $26.0^{\circ} \mathrm{C}$ at the bottom of the bed. The experiment was run until the bed was completely dry at approximately 191 hours. The liquid water in the warm upper region evaporated, diffused through the cold lower region, and then condensed in the bottom water tray. After 143.8 


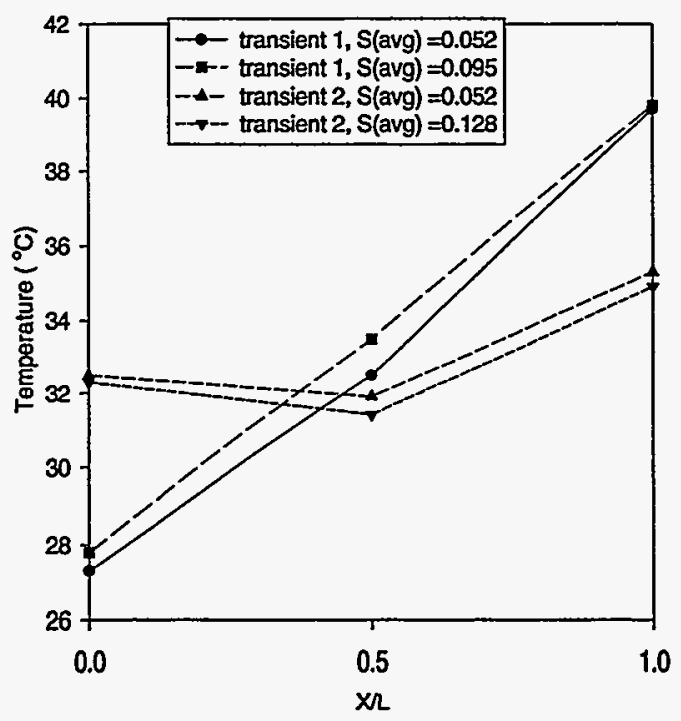

Figure 4-13

Packed Bed Temperature Profiles

hours, the lower bottom $10 \%$ of the bed contained some liquid near irreducible saturation. The experimental drying rates were quantified in two ways by the integration of the saturation distribution and by weighing the packed bed and the water tray. The results of these two methods agreed to within $\pm 7 \%$.

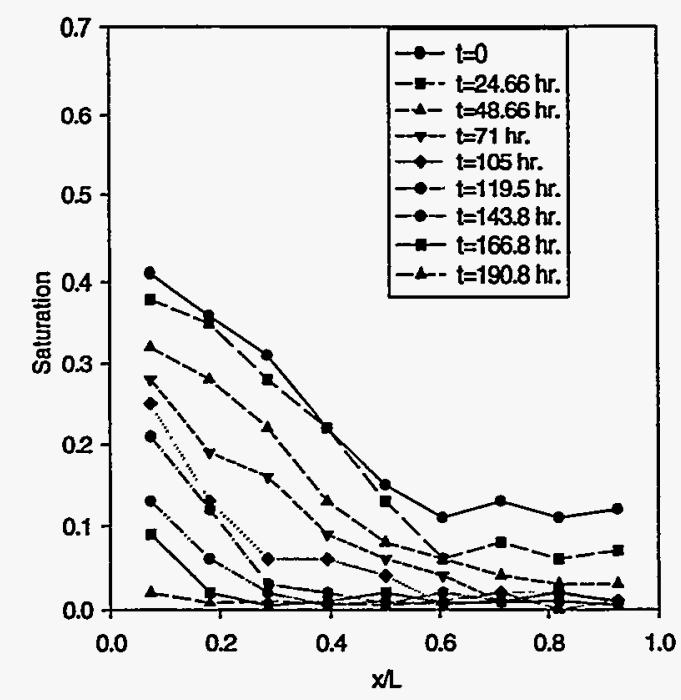

Figure 4-14

Saturation Profile vs. Time, Drying Experiment 
Nonisothermal experiments in packed beds of glass beads confirmed the existence of enhanced vapor diffusion resulting from pore-scale condensation and evaporation. The data from these experiments have been utilized to extract enhancement factors which depends on the average saturation in the bed. Although the experimental data is complicated by the transient nature of the experiment and nonuniform saturation distribution, the results are qualitatively similar to those reported previously. The measured enhancement factor defined as the effective diffusion coefficient normalized by the dry porous media diffusion coefficient increases from 1.0 to approximately 4.0 as the average saturation increases from 0.0 to 0.2 .

\subsection{Summary and Conclusions}

As part of the LDRD on Enhanced Vapor Diffusion (EVD) in Porous Media, experiments have been conducted to determine if EVD exists. The approach taken to answer this question is to experimentally measure EVD at three different length scales; a single pore, a fracture aperture, and a porous medium. These measurements are the first known direct data for enhanced vapor diffusion.

Pore-scale experiments have been conducted at New Mexico Tech to investigate EVD on an individual pore basis where the mechanisms involved are well controlled. These experimental results confirm the existence of EVD on the pore scale, which is a function of liquid island length. In addition, they demonstrate that EVD can occur due to a concentration gradient; a temperature gradient is not necessary. These results confirm the results of Webb and Ho (1997) and are contrary to the model of Philip and deVries (1957).

At Sandia, a fracture aperture setup consisting of two roughened glass plates is being used to look at EVD in two dimensions including the effects of isolated liquid zones. Preliminary experiments have been completed, and the final tests are underway.

Finally, porous media tests were conducted at Washington State University using a packed bed of glass beads. Significant EVD at the porous media scale was observed under transient and steady-state conditions.

In summary, it has been conclusively proven that enhanced vapor diffusion in porous media does exist, and that the enhancement factor may be a factor of 3 or more, which is consistent with the modeling results given earlier. 


\subsection{Discussion}

\subsection{General}

The modeling efforts in this report, including the development of the first mechanistic model, showed that EVD may occur and may be significant in the transport of vapor through porous media. Experimental measurements of EVD were conducted at the pore scale, in a twodimensional network - fracture aperture, and in a porous medium. These measurements, which are the first to directly measure enhanced vapor diffusion, support the modeling results in that EVD may be a significant transport mechanism for vapor transport in porous media. Note that the experimental section is not complete because final documentation is not yet available. When these reports are available, an addendum to this report will be issued.

\subsection{Definition of EVD Factors}

Comparison of the enhancement factors $\beta$ and $\eta$ from various investigators needs to be done carefully. The standard definition by Philip and deVries (1957) bases the factors on the thermally-induced vapor diffusion rate. In contrast, $\mathrm{Gu}$ (1998) base their enhancement factors on the total vapor diffusion rate (concentration plus temperature).

Note that the thermally-induced vapor diffusion rate from Philip and deVries (1957) is not the same as the thermal diffusion considered by Gu et al. (1998). Gu et al. (1998) calculated the total vapor diffusion rate from separate concentration and temperature gradients, similar to the procedure used in gas diffusion. Philip and deVries (1957) considered vapor diffusion due to a concentration gradient only and then proceeded to separate the concentration gradient into gradients of temperature and moisture content. As such, the temperature gradient includes the change in saturated vapor pressure with temperature for a constant relative humidity (Nakano and Miyazaki, 1979, corrected their expression to include the dependence of the relative humidity on temperature). The moisture content gradient term reflects the impact of vapor pressure lowering. Only the temperature gradient term was enhanced; the moisture content gradient term was not enhanced. Therefore, the enhancement factors of $\mathrm{Gu}$ should be smaller than those in previous studies which used the Philip and deVries (1957) model.

In the pore-scale experiments performed at New Mexico Tech, the vapor pressure was lowered through the use of salts rather than through the application of temperature and/or moisture content gradients. The model of Philip and deVries (1957) could not be expected to directly apply because the use of salts was not included. In any event, however, Philip and deVries (1957) assumed that a thermal gradient was necessary for enhancement. Even if they had considered a concentration gradient due to the use of salt solutions, they would not have enhanced that component. 


\subsection{Comparison of Modeling and Experimental Results}

The original two-dimensional enhancement results of Webb and Ho (1997), which are given in Figures 3-11 and 3-17 of this report, were presented as the enhancement as a function of liquid island length. For comparison to experimental data, these results have been converted to enhancement as a function of model saturation. The results for the two-dimensional single pore and pore network are shown in Figure 5-1. Also shown are the data of $\mathrm{Gu}$ et al. (1998), which were previously shown in Figure 4-9 of this report. The single pore results are considered to be the maximum enhancement factor because the liquid islands would be uniformly distributed among the pores. The pore network model with a single liquid island is the minimum enhancement factor. Note that the porosity of the model is 0.322 .

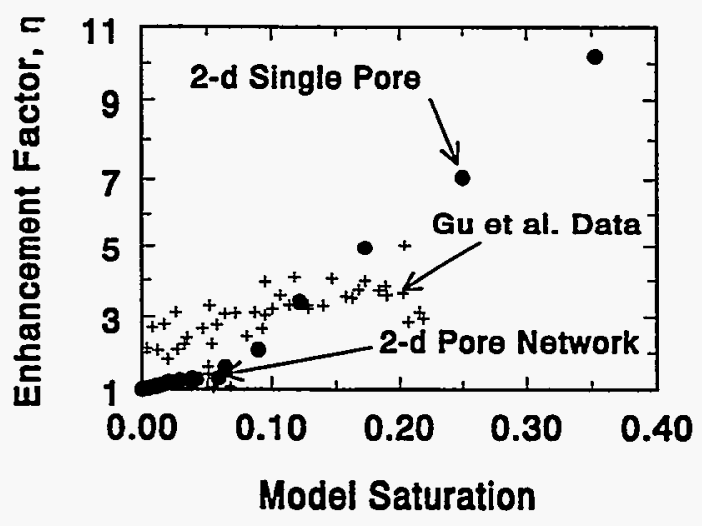

Figure 5-1

Experimental and Modeling Enhancement Factors, $\eta$

The enhancement factor in the model is applied to the total vapor diffusion rate similar to $\mathrm{Gu}$ et al. (1998) as discussed above. The calculated enhancement factor is in qualitative agreement with the data and model of Cass et al. (1984) and with the data of Gu et al. (1998). The enhancement factor starts out as 1.0, which slowly increases until a saturation of about 0.05 . The factor then increases rapidly, reaching a value of over 5.0 at a saturation of 0.20 .

The modeling and experimental results from this investigation provide additional mechanisms by which EVD can occur beyond those described by Philip and deVries (1957). In their model, EVD only enhances the vapor diffusion due to a thermal gradient; if there is no thermal gradient, EVD does not occur. Modeling and experimental data show that EVD may occur under isothermal conditions with only a concentration gradient. In fact, based on modeling results, the addition of an aiding temperature gradient actually reduces enhancement factor. The Philip and deVries model may need to be revised to reflect these findings. 


\subsection{Scaling Considerations}

The modeling results indicate that the mechanisms involved in EVD are probably well understood. At the pore-scale, no separate EVD factor is needed because the enhancement can be calculated mechanistically. However, as the scale of the domain and the size of the control volumes / elements increases compared to the characteristic dimension of the liquid islands, scaling factors such as $\beta$ or $\eta$ are needed to mimic the pore-scale processes involved.

Questions of upscaling the pore-scale results could be studied in conjunction with application of pore network schemes such that a realistic network of pores and liquid islands is considered. As shown in the modeling section, the resistance of the liquid islands can be neglected, and vapor diffusion in the gas portion of the model can be calculated to give an enhancement factor. These calculations can be performed using an electrical analogy similar to the model of Prat (1993), at least for the concentration gradient term. Although not shown in this report, unpublished results show that the simple model can also successfully predict the transient enhancement factors given in Section 3.2.3.2. Therefore, this approach could be used in a large pore network where detailed mechanistic modeling may be prohibitive.

\subsection{Vadose Zone Application}

The near-surface vadose zone is an area where EVD may have a significant impact. Using the model of plant-atmosphere-soil of Braud et al. (1995), Boulet et al. (1997) analyzed the near-surface vapor and liquid transport for a bare soil. Boulet et al. (1997) showed that vapor transfer dominates liquid water transport in the top $25 \mathrm{~cm}$ of the vadose zone near the surface. They also present the various driving forces for evaporation for noon on a given day. Surprisingly, the hotter surface temperatures drive vapor down into the soil, while potential gradients cause evaporation. They also present a variation of the vapor diffusivities (potential and thermal) due to the uncertainty regarding the values. The variation presented shows a significant influence of the diffusivities on the total evaporation from the soil.

Based on the results of Boulet et al. (1997) and those in the present LDRD, some additional calculations were made using the SiSPAT model of Braud et al. (1995) for the conditions analyzed by Boulet et al. (1997). The model and input data were obtained directly from Isabelle Braud (Braud, personal communication, 1998). Using the model and input data, evaporation over a specified 10 day period was calculated to be $2.51 \mathrm{~cm}$, which compares favorably to the value of $2.33 \mathrm{~cm}$ in the original paper. The differences are due to modifications in the code to correct minor errors (Braud, personal communication, 1998).

Coding changes were made to the SiSPAT program to analyze the same conditions with no enhancement. Surprisingly, the cumulative evaporation actually increased from 2.51 (enhancement) to $3.94 \mathrm{~cm}$ (no enhancement). Enhanced vapor diffusion using the Philip and 
deVries (1957) model actually decreases the net evaporation rate from bare soil, which seems counterintuitive.

Further modifications were made to enhance the total vapor flux due to potential and thermal gradients by a factor of $3(\eta=3 ; \beta \sim 0.7-0.8)$. The resulting cumulative evaporation increased from $3.94 \mathrm{~cm}$ (no enhancement) to $5.53 \mathrm{~cm}$ (enhancement), which seems appropriate; this compares to $2.51 \mathrm{~cm}$ with the Philip and deVries model. Intuitively, enhancement should increase the vapor diffusion and evaporation rate.

These results indicate that more study of EVD is needed, especially for modeling nearsurface vadose-zone evaporation. Based on the results of this LDRD, the Philip and deVries (1957) model may need to be modified. Based on some preliminary calculations of evaporation in the vadose zone, the modification of the Philip and deVries model may be important. 


\subsection{Summary and Conclusions}

An LDRD research project was conducted to answer the question of whether enhanced vapor-phase diffusion (EVD) in porous media exists. Based on a review of previous work, the existence of EVD was questioned. As a result, the LDRD developed the first mechanistic model of EVD and performed the first direct measurements of EVD. The important results and conclusions from this LDRD are as follows:

\subsection{Modeling Results}

1. The first mechanistic model of EVD has been developed. These models demonstrate the mechanisms responsible for EVD.

2. Significant enhancement of vapor diffusion is possible in the presence of liquid islands. The enhancement increases dramatically as the length of the liquid island increased.

3. A simple diffusion model assuming that the liquid island has zero resistance to diffusion gives reasonable results compared to detailed simulations.

4. The enhancement factor for vapor diffusion should be based on the average mass flux for transient and steady-state conditions.

5. Vapor pressure lowering should be considered in pore-scale analysis of vapor diffusion in the presence of its own liquid. While the magnitude of vapor pressure lowering may be small compared to the total vapor pressure, it can have significant impact on local vapor condensation, evaporation, and the evolution of liquid islands within the pores.

6. For enhanced vapor diffusion, the concentration gradient effect is predominant. The temperature gradient effect is of lesser importance.

While the present model indicates that enhanced vapor diffusion is possible, only experimental data can confirm the process. As noted by Ewing and Gupta (1993), pore-scale modeling is a "useful concept rather than a physical reality". The present authors support this notion.

\subsection{Experimental Results}

1. The first direct measurements of EVD have been conducted. Measurements were made at the pore scale, in a two-dimensional porous plate, and in a porous media.

2. Pore-scale experiments have been conducted at New Mexico Tech to investigate EVD on an individual pore basis. These experimental results confirm the existence of EVD on the pore scale, which is a function of liquid island length. In addition, they demonstrate that 
EVD can occur due to a concentration gradient; a temperature gradient is not necessary.

3. At SNL, a two-dimensional network - fracture aperture setup consisting of two roughened glass plates is being used to look at EVD in two dimensions including the effects of isolated liquid zones. Preliminary experiments have been completed, and the final tests are underway.

4. Porous media tests were conducted at Washington State University using a packed bed of glass beads. Significant EVD at the porous media scale was observed under transient and steady-state conditions.

\subsection{Overall}

1. The existence of enhanced vapor diffusion (EVD) has been confirmed. The existence of EVD and the mechanisms involved have been demonstrated by modeling and experimental programs within this LDRD.

2. The modeling and experimental results provide additional mechanisms for EVD beyond those presented by the generally accepted model of Philip and deVries (1957), which required a thermal gradient for EVD to exist. Modeling and experimental results show significant enhancement for a concentration gradient under isothermal conditions. Therefore, the model of Philip and deVries (1957) may need to be modified to include these additional mechanisms.

3. EVD may be significant in many areas including the near-surface vadose zone.

Calculations suggest that the cumulative evaporation rate is sensitive to the magnitude and application of the enhancement factors. If the enhancement factor is applied to just the thermal gradient, consistent with the Philip and deVries model, the evaporation actually decreases compared to no enhancement. In contrast, if the enhancement factor is applied to the total vapor flux, evaporation is significantly increased. This area needs to be investigated further.

4. In order to provide a solid foundation for analyzing vapor diffusion in porous media, a comparison of gas diffusion models was also conducted as summarized in Appendix A. The commonly-used advective-dispersive model is generally inadequate when gas diffusion is an important gas transport process. The Dusty-Gas model is much more accurate and follows fundamental gas diffusion principles as given by Graham's Laws. 


\section{Acknowledgments}

The authors would like to thank Bob Glass and Mike Itamura for their reviews of this report. We also appreciate the thought-provoking conversations with Kent Udell and Mike Itamura, then at UC-Berkeley, regarding the analytical pore-scale model. This work was supported by the United States Department of Energy under Contract DE-AC04-94AL85000 as part of a Sandia LDRD Project on Enhanced Vapor-Phase Diffusion in Porous Media. Sandia is a multiprogram laboratory operated by Sandia Corporation, a Lockheed Martin Company, for the United States Department of Energy.

\section{References}

Abriola, L.M., and Pinder, G.F., 1985, "A Multiphase Approach to the Modeling of Porous Media Contamination by Organic Compounds - 1. Equation Development," Water Resourc. Res., Vol. 21, pp. 11-18.

Adenekan, A.E., Patzek, T.W., and Pruess, K., 1993, "Modeling of Multiphase Transport of Multicomponent Organic Compounds and Heat in the Subsurface: Numerical Model Formulation," Water Resour. Res., Vol. 29, pp. 3727-3740.

Boulet, G., Braud,I., and Vauclin, M., 1997, "Study of the mechanisms of evaporation under arid conditions using a detailed model of the soil-atmosphere continuum. Application to the EFEDA I experiment," J. Hydrol., 193:114-141.

Braud, I., Dantas-Antonino, A.C., Vauclin, M., Thony, J.L., and Ruelle, P., 1995, "A simple soilplant-atmosphere transfer model (SiSPAT): development and field verification," J. Hydrol., 166:213-250.

Brown, S.R., Stockman, H.W. and Reeves, S.J., 1995, "Applicability of the Reynolds equation for modeling fluid flow between rough surfaces," Geo. Res. Let., 22:2537-2540.

Campbell, G,S., 1985, "Soil Physics with BASIC," Elsevier, Amsterdam.

Carman, P.C., 1952, "Diffusion and Flow of Gases and Vapours Through Micropores: IV. Flow of Capillary Condensate," Proc. R. Soc. London, A211, 526.

Carman, P.C., and Malherbe, P. le R., 1950, "Diffusion and Flow of Gases and Vapours Through Micropores: II. Surface Flow," Proc. R. Soc. London, A203, 165.

Carman, P.C., and Raal, F.A., 1951a, "Diffusion and Flow of Gases and Vapours Through Micropores: III. Surface Diffusion Coefficients and Activation Energies," Proc. R. Soc. London, A209, 38.

Carman, P.C., and Raal, F.A., 1951b, "Physical Adsorption of Gases on Porous Solids: I. Comparison of Loose Powder and Porous Plugs," Proc. R. Soc. London, A209, 59.

Cary, J.W., 1963, "Onsager's Relation and the Non-Isothermal Diffusion of Water Vapor," J. Phys. Chem., Vol. 67, pp. 126-129.

Cary, J.W., 1964, "An Evaporation Experiment and its Irreversible Thermodynamics," Int. J. Heat Mass Transfer, Vol. 7, pp. 531-538.

Cary, J.W., 1965, "Water Flux in Moist Soil: Thermal Versus Suction Gradients," Soil Science, 100:168-175. 
Cary, J.W., 1979, "Soil heat transducers and water vapor flow," Soil Sci. Soc. Am. J., 43:835839.

Cary, J.W. and Taylor, S.A., 1962a, "The Interaction of the Simultaneous Diffusions of Heat and Water Vapor," Soil Sci. Soc. Am. Proc., 26:413-416.

Cary, J.W. and Taylor, S.A.. 1962b, "Thermally Driven Liquid and Vapor Phase Transfer of Water and Energy in Soil," Soil Sci. Soc. Am. Proc., 26:417-420.

Cass, A., Campbell, G.S., and Jones, T.L., 1984, "Enhancement of Thermal Water Vapor Diffusion in Soil," Soil Sci. Soc. Am. J.,48:25-32.

Ceaglske, N.H. and Hougen, O.A., 1937, "Drying Granular Solids," Ind. Eng. Chem., 29:805813.

Clifford, S.M., 1991, "The Role of Thermal Vapor Diffusion in the Subsurface Hydrological Evolution of Mars," Geophysical Research Letters, 18:2055-2058.

Cunningham, R.E., and Williams, R.J.J., 1980, Diffusion in Gases and Porous Media, Plenum Press, New York.

de Marsily, G., 1986, Quantitative Hydrogeology Groundwater Hydrology for Engineers, Academic Press, Inc., San Diego.

Dullien, F.A.L., 1992. Porous Media Fluid Transport and Pore Structure, Second Edition, Academic Press, Inc., San Diego.

Evans, III, R.B., Watson, G.M., and Truitt, J., 1962, "Interdiffusion of gases in a low permeability graphite at uniform pressure," J. Appl. Phys., 33:2682-2688.

Evans, III, R.B., Watson, G.M., and Truitt, J., 1963, "Interdiffusion of gases in a low permeability graphite. II. Influence of pressure gradients," J. Appl. Phys., 34:2020-2026.

Ewing, R.P., and Gupta, S.C. 1993, "Modeling percolation properties of random media using a domain network," Water Resourc. Res., 29:3169-3178.

Falta, R.W., Pruess, K., Finsterle, S., and Battistelli, A., 1995, T2VOC User's Guide, LBL36400, Lawrence Berkeley Laboratory.

Finsterle, S., Schlueter, E., and Pruess, K., 1990, Exploratory Simulations of Multiphase Effects in Gas Injection and Ventilation Tests in an Underground Rock Laboratory, LBL-28810, Lawrence Berkeley Laboratory, Berkeley, CA, 1990.

Glass, R.J., and Nicholl, M.J., 1995, "Quantitative visualization of entrapped phase dissolution within a horizontal flowing fracture," Geophysical Research Letters, 22:11:1413-1416.

Graham, T., 1833, "On the law of the diffusion of gases," Phil. Mag., 2:175, 269, 351, reprinted in "Chemical and Physical Researches," 44-70, Edinburgh Univ. Press, Edinburgh, 1876.

Graham, T., 1846, "On the motion of gases, "Phil. Trans. Roy. Soc., 136:573, reprinted in "Chemical and Physical Researches," 44-70, Edinburgh Univ. Press, Edinburgh, 1876.

Gu, L., 1998, "An Experimental and Numerical Study of Vapor Diffusion in Porous Media," M.S. Thesis, School of Mechanical and Materials Engineering, Washington State University, Pullman, WA.

Gu, L., Ho, C.K., Plumb, O.A., and Webb, S.W., 1998, "Diffusion with Condensation and Evaporation in Porous Media," ASME Proceedings of the 7th AIAA/ASME Thermophysics and Heat Transfer Conference, HTD-Vol. 357-2, pp. 213-220. 
Gu, L., Ho, C.K., Plumb, O.A., and Webb, S.W., 1999, "Vapor Diffusion in Partially Saturated Packed Beds," submitted to the ASME/JSME Thermal Engineering Joint Conference, San Diego, Ca, March 15-19, 1999.

Ho, C.K., and Udell, K.S. 1992, "An experimental investigation of air venting of volatile liquid hydrocarbon mixtures from homogeneous and heterogeneous porous media," J. Contam. Hydrol., 11:291-316.

Ho, C.K., and Webb, S.W. 1996, A Review of Porous Media Enhanced Vapor-Phase Diffusion Mechanisms, Models, and Data - Does Enhanced Vapor-Phase Diffusion Exist? SAND961198, Sandia National Laboratories.

Ho, C.K., and Webb, S.W. 1998, "A Review of Porous Media Enhanced Vapor-Phase Diffusion Mechanisms, Models, and Data - Does Enhanced Vapor-Phase Diffusion Exist?" J. Porous Media, 1:71-92.

Hsu, C.T., Cheng, P., and Wong, K.W., 1995. "A Lumped-Parameter Model for Stagnant Thermal Conductivity of Spatially Periodic Porous Media," Trans. ASME, J. Heat Transfer, 117:264-269

Jackson, R.. 1977, Transport in Porous Catalysts, Chem Eng. Monograph 4, Elsevier, New York.

Jury, W.A., and Letey, Jr., J., 1979, "Water Vapor Movement in Soil: Reconciliation of Theory and Experiment," Soil Sci. Soc. Am. J., 43:823-827.

Lebedev, P. D., 1961, "Heat and Mass Transfer during the Drying of Moist Materials," Int. J. Heat Mass Transfer, 1:37-45.

Lee, K.-H., and Hwang, S.-T., 1986, "The Transport of Condensible Vapors through a Microporous Vycor Glass Membrane," J. Colloid and Interface Science, 110:544-555.

Lenhard, R.J., Oostrom, M., Simmons, C.S., and White, M.D., 1995, "Investigation of densitydependent gas advection of trichloroethylene: Experiment and a model validation exercise," $\mathrm{J}$. Contaminant Hydrology, Vol. 19, pp. 47-67.

Luikov, A. V., 1975, "Systems of Differential Equations of Heat and Mass Transfer in CapillaryPorous Bodies (Review)," Int. J. Heat Mass Transfer, 18:1-14.

Martinez, M.J., 1995a, Formulation and Numerical Analysis of Nonisothermal Multiphase Flow in Porous Media, SAND94-0379, Sandia National Laboratories.

Martinez, M.J., 1995b, Mathematical and Numerical Formulation of Nonisothermal Multicomponent Three-Phase Flow in Porous Media, SAND95-1247, Sandia National Laboratories.

Martinez, M.J., Hopkins, P.L., and Shadid, J.N., 1997, LDRD Final Report: Physical Simulation of Nonisothermal Multiphase Multicomponent Flow in Porous Media, SAND97-1766, Sandia National Laboratories.

Mason, E.A., and Malinauskas, A.P., 1983, Gas Transport in Porous Media: The Dusty-Gas Model, Chem Eng. Monograph 17, Elsevier, New York.

Mikhailov, M. D., 1973, "General Solutions of the Diffusion Equations Coupled at Boundary Conditions," Int. J. Heat Mass Transfer, 16:2155-2164.

Milly, P.C.D., 1982, "Moisture and Heat Transport in Hysteretic, Inhomogeneous Porous Media: A Matric Head-Based Formulation and a Numerical Model," Water Resour. Res., 18:489-498. Milly, P.C.D., 1984a, "A Linear Analysis of Thermal Effects on Evaporation From Soil," Water Resour. Res., 20:1075-1085. 
Milly, P.C.D., 1984b, "A Simulation Analysis of Thermal Effects on Evaporation From Soil," Water Resour. Res., 20:1087-1098.

Milly, P.C.D., 1996, "Effects of thermal vapor diffusion on seasonal dynamics of water in the unsaturated zone," Water Resour. Res., 32:509-518.

Nakano, M., and Miyazaki, T., 1979, "The Diffusion and Nonequilibrium Thermodynamic Equations of Water Vapor in Soils Under Temperature Gradients," Soil Science, 128:184-188.

Nassar, I.N., and Horton, R., 1997, "Heat, Water, and Solute Transfer in Unsaturated Porous Media:I-Theory Development and Transport Coefficient Evaluation," Trans. in Porous Media, 27:17-38.

Nicholl, M.J., Glass, R.J., and Wheatcraft, S.W., 1994, "Gravity-driven infiltration instability in initially dry non-horizontal fractures," Water Resources Research, 30:9:2533-2546.

Nowicki, S.C., Davis, H.T., and Scriven, L.E., 1992, "Microscopic Determination of Transport Parameters in Drying Porous Media," Drying Technology, 10:925-946.

Nozad, I., Carbonell, R.G., and Whitaker, S., 1985, "Heat Conduction in Multiphase Systems - I: Theory and Experiment for Two-Phase Systems," Chem. Eng. Sci., Vol. 48, No. 5, pp. 843855.

Philip, J.R., and deVries, D.A., 1957, "Moisture Movement in Porous Materials under Temperature Gradients," Trans., Am. Geophys Union, Vol. 38, No. 2, pp. 222-232, p. 594.

Plumb, O.A., and Prat, M., 1992, "Microscopic Models for the Study of Drying of Capillary Porous Media," Drying '92, pp. 397-406.

Prat, M., 1993, "Percolation Model of Drying Under Isothermal Conditions in Porous Media,", Int. J. Multiphase Flow, 19:691-704.

Pruess, K., 1987, TOUGH User's Guide, LBL-20700, Lawrence Berkeley Laboratory, Berkeley, CA.

Pruess, K., 1991a, TOUGH2 - A General-Purpose Numerical Simulator for Multiphase Fluid and Heat Flow, LBL-29400, Lawrence Berkeley Laboratory.

Pruess, K., 1991b, "Grid Orientation and Capillary Pressure Effects in the Simulation of Water Injection into Depleted Vapor Zones," Geothermics, 20:257-277.

Pruess, K., 1995, Proceedings of the TOUGH Workshop '95, LBL-37200, Lawrence Berkeley Laboratory.

Pruess, K., 1998, Proceedings of the TOUGH Workshop '98, LBL-41995, Lawrence Berkeley National Laboratory.

Pruess, K., and Bodvarsson, G.S., 1983, "A Seven-Point Finite Difference Method for Improved Grid Orientation Performance in Pattern Steamfloods," Paper SPE-12252, Seventh Society of Petroleum Engineers Symposium on Reservoir Simulation, San Francisco, CA.

Pruess, K., and Narasimhan, T.N., 1985, "A Practical Method for Modeling Fluid and Heat Flow in Fractured Porous Media," SPE Journal, 25:14-26.

Pruess K. and Tsang, Y., 1993, Modeling of Strongly Heat-Driven Flow Processes at a Potential High-Level Nuclear Waste Repository at Yucca Mountain, Nevada, LBL-33597, Lawrence Berkeley Laboratory, Berkeley, CA.

Rajniak, P., and Yang, R.T., 1996, "Unified Network Model for Diffusion of Condensible Vapors in Porous Media," AlChE J., 42:319-331. 
Ryan, D., Carbonell, R.G., and Whitaker, S., 1981, "A Theory of Diffusion and Reaction in Porous Media," AlChE Symposium Series, 202(77), pp. 46-62.

Sahraoui, M., and Kaviany, M., 1993, "Slip and no-slip temperature boundary conditions at interface of porous, plain media: conduction," Int. J. Heat Mass Trans., Vol. 36, No. 4, pp. 1019-1033.

Shonnard, D.R., and Whitaker, S., 1989, "The effective thermal conductivity for a point-contact porous medium: an experimental study," Int. J. Heat Mass Trans., Vol. 32, No. 3, pp. 503-512.

Shurbaji, A.-R.M., and Phillips, F.M., 1995, "A numerical model for the movement of $\mathrm{H}_{2} \mathrm{O}, \mathrm{H}_{2}{ }^{18} \mathrm{O}$, and ${ }^{2} \mathrm{HHO}$ in the unsaturated zone," J. Hydrology, 171:125-142.

Sotirchos, S.V., and Burganos, V.N., 1988, "Analysis of Multicomponent Diffusion in Pore Networks," AIChE J., 34:1106-1118.

Steele, D.D., and Nieber, J.L., 1994a, "Network Modeling of Diffusion Coefficients for Porous

Media: I. Theory and Model Development," Soil Sci. Soc. Am. J., 58:1337-1345.

Steele, D.D., and Nieber, J.L., 1994b, "Network Modeling of Diffusion Coefficients for Porous Media: II. Simulations," Soil Sci. Soc. Am. J., 58:1346-1354.

Thorstenson, D.C., and Pollock, D.W., 1989, "Gas Transport in Unsaturated Zones:

Multicomponent Systems and the Adequacy of Fick's Laws" Water Resour. Res., 25:477-507. Tsang, Y.W., and Pruess, K., 1990, Further Modeling Studies of Gas Movement and Moisture Migration at Yucca Mountain, Nevada, LBL-29127, Lawrence Berkeley Laboratory. Webb, S.W., 1996, Gas-Phase Diffusion in Porous Media - Evaluation of an AdvectiveDispersive Formulation and the Dusty-Gas Model Including Comparison to Data for Binary Mixtures, SAND96-1197, Sandia National Laboratories.

Webb, S.W., 1998a, "Pore-Scale Modeling of Transient and Steady-State Vapor Diffusion in Partially-Saturated Porous Media," ASME Proceedings of the 7th AIAA/ASME Thermophysics and Heat Transfer Conference, HTD-Vol. 357-2, pp. 221-231.

Webb, S.W., 1998b, "Gas-Phase Diffusion in Porous Media - Evaluation of an AdvectiveDispersive Formulation and the Dusty-Gas Model," J. Porous Media, 1:187-199.

Webb, S.W., 1998c, "Gas-Phase Diffusion in Porous Media: Comparison of Models," Proceedings of the TOUGH Workshop '98, LBNL-41995, pp. 269-274.

Webb, S.W., 1999, "Temperature Gradient Effects in Vapor Diffusion in Partially-Saturated Porous Media," submitted to the ASME/JSME Thermal Engineering Joint Conference, San Diego, Ca, March 15-19, 1999.

Webb, S.W., and Ho, C.K., 1997, "Pore-Scale Modeling of Enhanced Vapor Diffusion in Porous Media," Proceedings of the ASME Fluids Engineering Division, FED-Vol. 244, pp. 457-468.

Webb, S.W., and Phelan, J.M., 1997, "Effect of Soil Layering on NAPL Removal Behavior in Soil-Heated Vapor Extraction," J. Contam. Hydrol., 27:285-308.

Weeks, L.V., Richards, S.J., and Letey, J., 1968, "Water and Salt Transfer in Soil Resulting from Thermal Gradients," Soil Sci. Soc. Am. Proc., 32:193-197.

Weisz, P.B., 1975, "Diffusion Transport in Chemical Systems - Key Phenomena and Criteria," Ber. Bunsenges, Phys. Chem., 79:798-806.

Whitaker, S., 1977, "Simultaneous heat, mass and momentum transfer in porous media. A theory of drying." Advances in Heat Transfer, Vol. 13, Academic Press, New York. 


\section{Appendix A \\ Comparison of Gas Diffusion Models}

\section{A.1 Introduction}

In order to provide a solid foundation for the analysis of vapor diffusion, a survey of prediction methods used to predict gas diffusion was conducted. The Advective-Dispersive model is commonly used in porous media applications in Mechanical Engineering and Hydrology. The model simply adds the flux due to advection to that due to diffusion. The second model is the Dusty Gas Model (DGM), which is widely used in Chemical Engineering. Based on this survey, Webb $(1996,1998 \mathrm{~b}, 1998 \mathrm{c})$ compared the two models to each other and to some experimental data. This section is essentially the summary paper by Webb (1998c). Webb $(1996,1998 b)$ contains additional material on the subject.

Gas-phase diffusion in porous media may be significant in the flow of multiphase fluids and the transport of contaminants in the subsurface. In many applications, such as removal of nonaqueous phase liquid (NAPL) contaminants from low-permeability layers in the subsurface, gas-phase diffusion may be the limiting transport mechanism (Ho and Udell, 1992, Webb and Phelan, 1997). Gas-phase diffusion may also be important in the analysis of contaminant migration and/or drying processes in potential nuclear waste repositories such as Yucca Mountain (Pruess and Tsang, 1993; Finsterle, Schlueter, and Pruess, 1990; Tsang and Pruess, 1990), in evaporation and drying in the vadose zone, and in porous catalysts (Jackson, 1977).

A number of different models for combined advection-diffusion of gases in porous media have been employed. The most widely used model is the Advective-Dispersive Model, or ADM, which is based on a linear addition of advection calculated by Darcy's Law and ordinary diffusion using Fick's Law. Knudsen diffusion is often included through the use of a Klinkenberg factor for advection, while the effect of a porous medium on the diffusion process is through a porositytortuosity-gas saturation multiplier. This model is employed in TOUGH2 (Pruess, 1991), a widely-used code for simulating flow and transport in fractured and porous media in nuclear waste, environmental, and geothermal applications (Pruess, 1995), the PNL code STOMP (Lenhard et al., 1995), and has been proposed as the model for the new Sandia massively parallel porous media code (Martinez, 1995a,b, Martinez et al., 1997). A number of other investigators, including Abriola and Pinder (1985), have used a similar approach. Note that the ADM is also used in T2VOC (Falta et al., 1995) and $M^{2}$ NOTS (Adenekan et al., 1993) for multicomponent diffusion transport of NAPLs (nonaqueous phase liquids) and VOCs (volatile organic compounds) along with water vapor and air because both of these codes are based on TOUGH2.

Another, more comprehensive approach for gas-phase transport in porous media has been formulated by Evans and Mason in a series of papers between 1961 and 1967, which is referred to as the Dusty-Gas Model (DGM). This model applies the kinetic theory of gases to the gaseous components and the porous media (or "dust") to develop an approach for combined transport due to ordinary and Knudsen diffusion and advection including porous medium effects. 
These two models are dramatically different in their treatment of gas-phase diffusion. The main objective of this section present paper is to present a comparison of the ADM and DGM models with Graham's laws, which are fundamental relationships for diffusion, and to evaluate both models against a number of comprehensive experimental data sets. Webb (1996, 1998a, 1998b) presents an additional comparison for an air-water heat pipe that is encountered in earth science applications.

\section{A.2 Diffusion Models}

\section{A.2.1 Advective-Dispersive Model}

The Advective-Dispersive Model (ADM) is commonly used in earth sciences as exemplified by TOUGH2 (Pruess, 1991). The ADM is based on a simple ad hoc linear addition of advection calculated by Darcy's Law and ordinary diffusion using Fick's Law. Slip effects, or Knudsen diffusion, are included through a Klinkenberg parameter to define the effective permeability for the advective mass flux. Porous medium effects for ordinary diffusion are included through a porosity-tortuosity-gas saturation factor on the diffusive mass flux in free space. This simple additive approach, while intuitively appealing, ignores possible coupling between advection and diffusion.

The ADM mass flux for component $I, F_{i}$, in a binary mixture

$$
F_{i}=-k \frac{k_{r, g}}{\mu} \rho_{g} \omega_{i}\left(\nabla P_{g}-\rho_{g} g_{c}\right)-D_{12} \rho_{g} \nabla \omega_{i}
$$

where $k$ is the permeability, $\mu$ is the viscosity, $P_{g}$ is the gas pressure, $\omega$ is the mass fraction, $\rho$ is the density, $g_{c}$ is the gravitational constant, and $D_{12}$ is the effective diffusion coefficient, and $\mathrm{D}_{12}=\mathrm{D}_{21}$ is assumed. The first term on the right hand side is the advective mass flux and the second term is the diffusive mass flux. Slip, or Knudsen diffusion, is simply incorporated into the model through modification of the permeability by the Klinkenberg factor, $b$, as

$$
k=k_{0}\left(1+b / P_{g}\right)
$$

where $\mathrm{k}_{0}$ is the intrinsic permeability and $\mathrm{P}_{\mathrm{g}}$ is the gas pressure.

The effective diffusion coefficient is a function of pressure and temperature and porous media parameters, or 


$$
D_{12}=\tau \phi S_{g} D_{12}^{0} \frac{P_{0}}{P}\left(\frac{T+273.15}{273.15}\right)^{\theta}
$$

where $\tau$ is the tortuosity, $\varphi$ is the porosity, $S_{g}$ is the gas saturation, $D_{12}{ }^{0}$ is the binary diffusion coefficient at $1 \mathrm{bar}\left(10^{5} \mathrm{~Pa}\right)$ and $0^{\circ} \mathrm{C}$ in free space, $\mathrm{P}_{0}$ is equal to $1 \mathrm{bar}, \mathrm{T}$ is the temperature in ${ }^{\circ} \mathrm{C}$, and the exponent $\theta$ is typically 1.8 (Pruess, 1987).

In the case of pure ordinary diffusion (no advection), the ADM reduces to

$$
\begin{aligned}
& F_{i=1}=-D_{12} \rho_{g} \nabla \omega_{i=1} \\
& F_{i=2}=-D_{21} \rho_{g} \nabla \omega_{i=2} .
\end{aligned}
$$

For a binary mixture, $\nabla \omega_{i=1}=-\nabla \omega_{i=2}$, and $D_{12}=D_{21}$, so

$$
F_{i=1}=F_{i=2},
$$

and the mass fluxes of the two components are equal and opposite.

\section{A.2.2 Dusty-Gas Model}

The Dusty-Gas Model (DGM) was developed to describe gas transport through porous media including the coupling between the various transport mechanisms. The term dusty-gas is used because the porous medium consists of large "dust" molecules fixed in space that are treated as a component of the gas mixture. The kinetic theory of gases is applied to this dusty-gas mixture. The DGM, including numerous data-model comparisons, is discussed in detail by Mason and Malinauskas (1983) and by Cunningham and Williams (1980). The present discussion is limited to a binary gas mixture. In addition, temperature gradients and external forces are ignored. The more complex nonisothermal and/or multicomponent system is treated in the DGM references given above.

Similar to the ADM. the DGM considers advection, Knudsen diffusion, and ordinary diffusion. One of the key aspects of the DGM is the combination of the diffusion (ordinary and Knudsen) and advection. Ordinary and Knudsen diffusion are combined through addition of momentum transfer based on kinetic-theory arguments, and diffusive fluxes (ordinary plus Knudsen) are added to advective fluxes based on Chapman-Enskog kinetic theory. 
There are many alternative forms of the DGM which can be derived by combining various forms of the equations. Thorstenson and Pollock (1989) present a number of useful forms, including specific forms for binary systems which is of special interest here. In particular, using the above equations and some patience, the total flux of component 1 in an isothermal binary system can be written as

$$
J_{1}=-\frac{\left[D_{1 K} D_{12} \frac{P_{g}}{R T} \nabla x_{1}+D_{1 K}\left(D_{12}+D_{2 K}\right) x_{1} \frac{\nabla P_{g}}{R T}\right]}{\left(D_{12}+x_{1} D_{2 K}+x_{2} D_{1 K}\right)}-x_{1}
$$

and the total mole flux is given by

$$
J=J_{1}+J_{2}=\frac{\left[D_{12}\left(D_{2 K}-D_{1 K}\right) \frac{P_{g}}{R T} \nabla x_{1}-\left(D_{12}\left(x_{1} D_{1 K}+x_{2} D_{2 K}\right)+D_{1 K}\right.\right.}{\left(D_{12}+x_{1} D_{2 K}+x_{2} D_{1 K}\right)}
$$

In equation (A-7), the flux of component 1 consists of a diffusive flux (first term) and an advective flux (second term). The diffusive flux has ordinary diffusion (mole fraction gradient) and Knudsen diffusion (pressure gradient) components similar to the ADM equation.

\section{A.2.3 ADM-DGM Comparison}

The predictions for both models were made by the TOUGH2 code (Pruess, 1991). The code was modified to use the DGM gas transport equations.

\section{A.2.3.1 Comparison to Fundamental Relationships}

Two important relationships were discovered by Thomas Graham in the 1800's for gas diffusion in a binary mixture (Mason and Malinauskas, 1983). For Knudsen diffusion, or freemolecule flow, Graham's law of effusion applies as experimentally discovered by Graham (1846). Similarly, for ordinary diffusion without advection, the ratio of the diffusive fluxes is given by Graham's law of diffusion as experimentally discovered by Graham (1833). The ratio of diffusive mole fluxes for both regimes is the same, or

$$
-J_{1 K} / J_{2 K}=-J_{1 D} / J_{2 D}=\left(M_{2} / M_{1}\right)^{1 / 2}
$$

while the diffusive mass flux ratio is 


$$
-F_{1 K} / F_{2 K}=-F_{1 D} / F_{2 D}=\left(M_{1} / M_{2}\right)^{1 / 2}
$$

The above relationships are very important. Graham's laws were misunderstood and were independently rediscovered in the 1950's and 1960's (Mason and Malinauskas, 1983). Graham's laws are fundamental relationships for gas diffusion and were used in the development of the DGM. The data of Evans, Watson, and Truitt $(1962,1963)$ strongly support Graham's laws and show that the assumption of equimolar counterdiffusion is not correct.

\section{A.2.3.2 Evans, Watson, and Truitt Data-Model Comparison}

Evans, Watson, and Truitt $(1962,1963)$ performed a number of experiments for flow and diffusion of helium and argon across low-permeability graphite. Pure helium was present on one side, while pure argon was present on the other. These data were obtained to support the DGM as summarized by Mason and Malinauskas (1983).

\section{A.2.3.2.1 Zero Pressure Difference}

The first data-model comparison considers zero pressure difference such that there is only diffusion. From Graham's laws, if only diffusion is occurring, the ratio of the mole fluxes (not the magnitude) for Knudsen diffusion and for ordinary diffusion is the same. Based on the molecular weights of helium $(M=4.00)$ and argon $(M=39.944)$, Graham's Laws give a mole flux ratio of helium to argon of 3.2.

Figure A-1 compares the data with the predictions from the $A D M$ and the $D G M$ as a function of the average pressure. Helium mole flux is positive, while argon mole flux is negative. The mole flux data for both gases increase with increasing pressure. The DGM predicts the variation of the data quite well. The ratio of the mole fluxes is consistent with the theoretical ratio given above. In contrast, the ADM predicts a constant mole flux value independent of pressure that is inconsistent with the data. As discussed earlier, for pure diffusion, the mass fluxes of the two components are equal in the current implementation of the ADM. The ADM mole flux ratio (helium/argon) is simply the inverse of the ratio of the molecular weights, or about 10; this ratio is much higher than the data. The ADM overpredicts the helium mole flux and underpredicts the argon mole flux. The ADM, which in this case is simply Fick's law, does not predict the correct gas diffusion values or trends for this simple diffusion-only case.

The constant flux prediction for the ADM can be explained by analyzing the formulation. In the ADM, only ordinary diffusion is important for zero pressure difference conditions; Knudsen diffusion is not invoked through the Klinkenberg factor because there is no pressure difference and no advective flow. For the ADM, the mass flux due to ordinary diffusion was given earlier where the diffusion coefficient, $D_{12}$, is inversely proportional to pressure. The gas density increases with pressure while the diffusion coefficient decreases with pressure. Therefore, for pure diffusion of perfect gases, the ADM will predict a constant mass and mole flux as a function of pressure. 


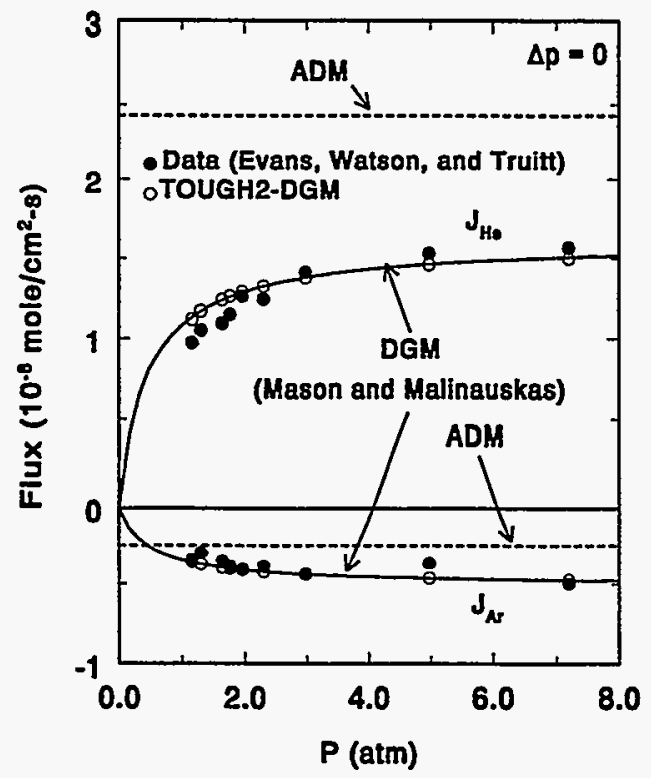

Figure A-1

Mole Flux Data-Model Comparison for Zero Pressure Difference Conditions

\section{A.2.3.2.2 Zero Net Mole Flux}

The second case simulates what would occur in a closed volume, where the total mole fluxes of the helium and argon components are equal, $\mathrm{J}_{\mathrm{He}}=-\mathrm{J}_{\mathrm{Ar}}$. In this situation, diffusion and advection balance each other resulting in a zero net mole flux. The predicted flux corresponding to this condition, and the pressure difference across the low-permeability graphite associated with it, are compared to the experimental data.

Figure A-2 shows the predicted mole flux for the ADM and the DGM along with the data as a function of the average pressure. The DGM predictions compare reasonably well with the experimental data including the variation of flux with pressure. In contrast, the ADM predicts a constant mole flux as a function of pressure similar to the zero pressure difference case. The ADM predictions are about $20 \%$ to $50 \%$ too high depending on the pressure; the higher error is for the lower pressures.

Figure A-3 presents the data for the pressure difference across the low-permeability graphite sample and the model predictions. The pressure difference is the value required for equal and opposite mole fluxes across the graphite. The data are well predicted by the DGM. The DGM predicts a maximum pressure difference at an average pressure of about 1 atmosphere; the data are not sufficient to resolve the details of this behavior. The shape of the DGM pressure difference 


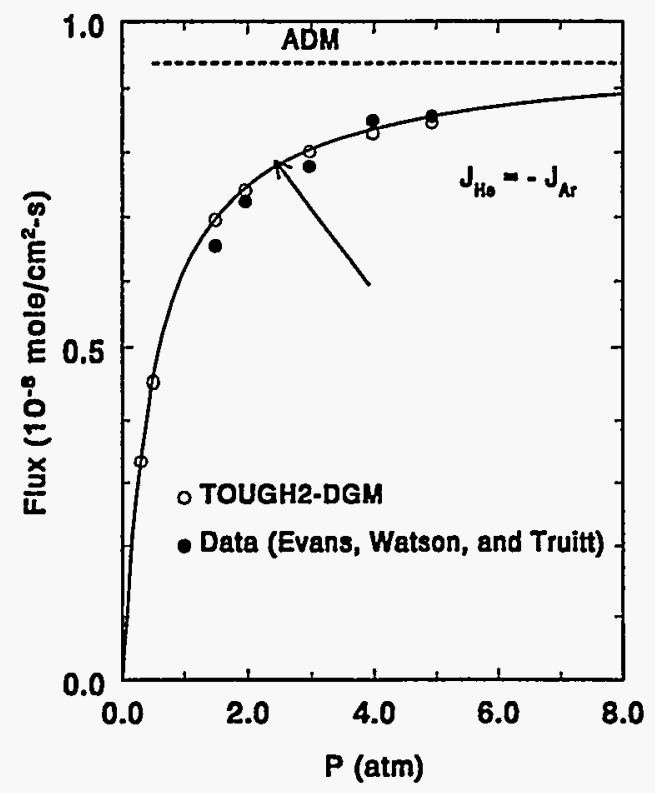

Figure A-2

Mole Flux Data-Model Comparison for Zero Net Mole Flux Conditions

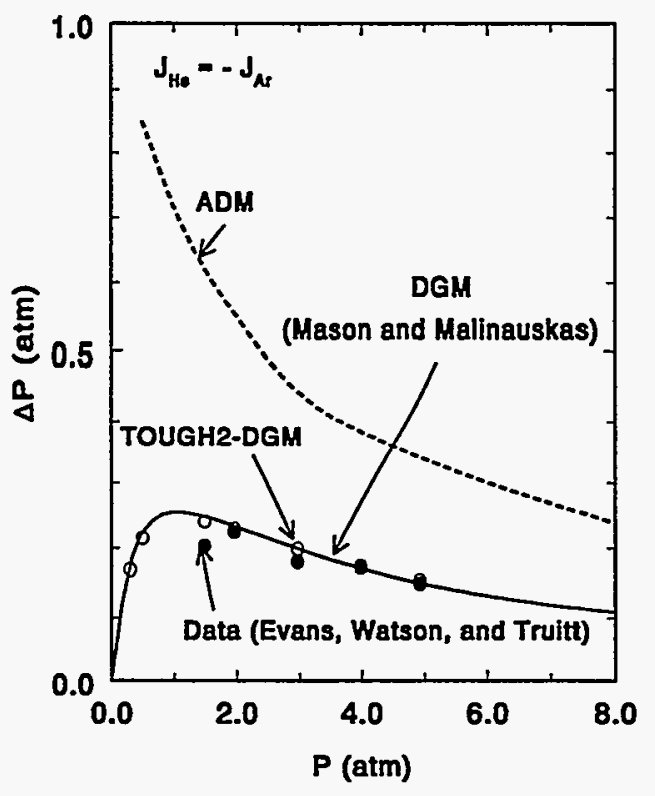

Figure A-3

Pressure Difference Data-Model Comparison For Zero Net Mole Flux Conditions

curve is due to the dominance of Knudsen diffusion pressure drop at low pressures and the dominance of advection pressure drop at higher pressures. The ADM consistently overpredicts the pressure difference by a factor of 2 or more and does not have the predicted shape of the DGM.

\section{A.2.3.2.3 Combined Advection and Diffusion}

The next case is the most general situation of combined advection and diffusion. In this series of tests, the pressure difference across the test section was varied while maintaining the same average pressure. The individual fluxes of helium and argon across the low-permeability graphite test section were recorded at steady-state conditions. The predicted mole fluxes as a function of pressure difference are compared in Figures A-4 and A-5. Figure A-4 presents the results for the ADM. The ADM predictions do not compare favorably with the data. Errors of a factor of 2 and greater are noted for the individual fluxes with corresponding differences in the net flux. While the general behavior is observed, the detailed variation is not reproduced by the ADM approach. In Figure A-5, DGM predictions of the mole fluxes are shown. These results compare quite well with the data for the individual mole fluxes of helium and argon as well as with the net flux, including the variation with pressure difference. 


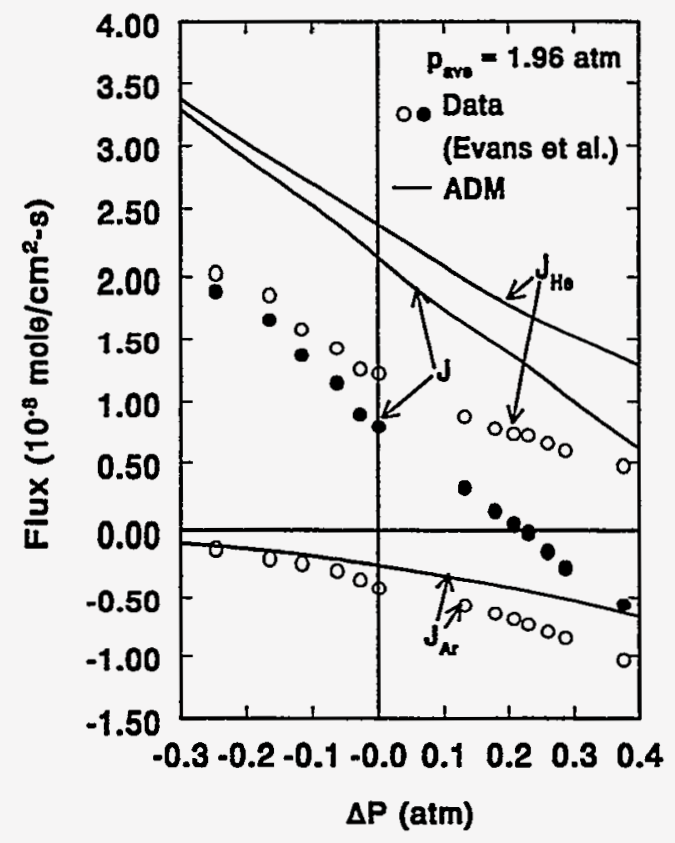

Figure A-4

ADM Mole Flux Data-Model Comparison for Combined Advection and Diffusion

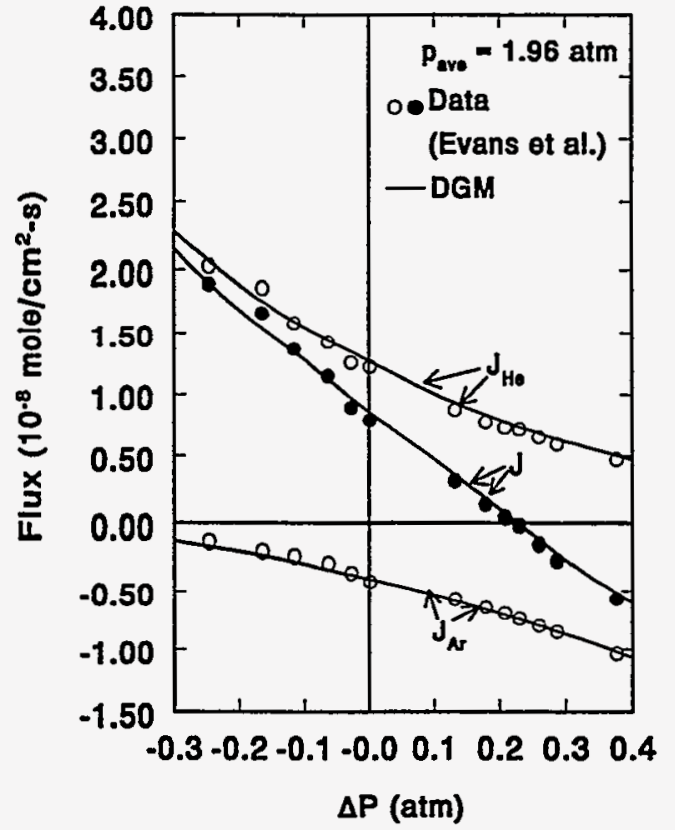

Figure A-5

DGM Mole Flux Data-Model Comparison for Combined Advection and Diffusion

\section{A.3 Discussion}

Two commonly used models for gas-phase diffusion and advection in porous media, the Advective-Dispersive Model (ADM) and the Dusty-Gas Model (DGM), have been compared. Evaluation of the models against Graham's laws and He-Ar data dramatizes the significant differences between the models. The DGM should, and does, agree very well with Graham's laws and the He-Ar data because both were used in the model development and validation of the DGM. In contrast, the ADM does not agree well with either Graham's laws or the experimental data. The magnitude and the trend of the ADM predictions, such as the mole flux variation with pressure for pure diffusion, are significantly different than the data. In this case, the DGM is significantly better than the ADM, which may give incorrect trends and/or misleading results. Webb $(1996,1998)$ provides more details on the comparison on these two models, including parameter values and additional cases. 


\section{A.4 Conclusions}

1. The ADM and DGM both consider advection, Knudsen diffusion, and ordinary diffusion. While the advective flow component can be shown to be the same for both models, significant differences may exist for the diffusion fluxes.

2. The DGM observes the fundamental relationships for diffusive fluxes given by Graham's laws. In contrast, the ADM violates these fundamental relationships.

3. DGM predictions are in excellent agreement with the comprehensive experimental $\mathrm{He}-\mathrm{Ar}$ data of Evans, Watson, and Truitt $(1962,1963)$. ADM predictions are in disagreement with the magnitude and some of the trends of the data.

4. The common conception of equimolar counterdiffusion is not supported by Graham's laws or by the He-Ar data of Evans, Watson, and Truitt (1962, 1963).

5. If diffusion is an important transport mechanism, the DGM is significantly better than the $\mathrm{ADM}$, although the ADM may give reasonable qualitative results. For quantitative analysis and the analysis of diffusion trends, the DGM should be used because the ADM may give incorrect trends and/or misleading results. 


\section{Appendix B \\ List of Reports and Papers}

A list of the SAND Reports, journal papers, and conference papers produced as part of this LDRD is given below. Additional papers are also in preparation.

\section{SAND Reports}

Ho, C.K., and Webb, S.W. 1996, A Review of Porous Media Enhanced Vapor-Phase Diffusion Mechanisms, Models, and Data - Does Enhanced Vapor-Phase Diffusion Exist?, SAND961198, Sandia National Laboratories.

Webb, S.W., 1996, Gas-Phase Diffusion in Porous Media - Evaluation of an AdvectiveDispersive Formulation and the Dusty-Gas Model Including Comparison to Data for Binary Mixtures, SAND96-1197, Sandia National Laboratories.

\section{Journal Papers}

Ho, C.K., and Webb, S.W. 1998, "A Review of Porous Media Enhanced Vapor-Phase Diffusion Mechanisms, Models, and Data - Does Enhanced Vapor-Phase Diffusion Exist?" J. Porous Media, 1:71-92.

Webb, S.W., 1998, "Gas-Phase Diffusion in Porous Media - Evaluation of an AdvectiveDispersive Formulation and the Dusty-Gas Model," J. Porous Media, 1:187-199.

Webb, S.W., and Phelan, J.M., 1997, "Effect of Soil Layering on NAPL Removal Behavior in Soil-Heated Vapor Extraction," J. Contam. Hydrol., 27:285-308.

\section{Conference Papers}

Gu, L., Ho, C.K., Plumb, O.A., and Webb, S.W., 1998, “Diffusion with Condensation and Evaporation in Porous Media," ASME Proceedings of the 7th AIAA/ASME Thermophysics and Heat Transfer Conference, HTD-Vol. 357-2, pp. 213-220.

Gu, L., Ho, C.K., Plumb, O.A., Webb, S.W., 1999, "Diffusion Dominated Drying of Packed Beds at Low Liquid Saturation," submitted to the ASME/JSME Thermal Engineering Joint Conference, San Diego, Ca, March 15-19, 1999.

Ho, C.K., and Webb, S.W. 1996, "A Review of Porous Media Enhanced Vapor-Phase Diffusion Mechanisms, Models, and Data - Does Enhanced Vapor-Phase Diffusion Exist?" Proceedings of the International Conference on Porous Media and Their Applications in Science, Engineering and Industry, pp. 173-198, June 16-21, 1996

Webb, S.W., 1996, "Gas-Phase Diffusion in Porous Media - Evaluation of an AdvectiveDispersive Formulation and the Dusty-Gas Model," Proceedings of the International 
Conference on Porous Media and Their Applications in Science, Engineering and Industry, pp. 559-599, June 16-21, 1996.

Webb, S.W., and Ho, C.K., 1997, "Pore-Scale Modeling of Enhanced Vapor Diffusion in Porous Media," Proceedings of the ASME Fluids Engineering Division, FED-Vol. 244, pp. 457-468.

Webb, S.W., 1998, "Pore-Scale Modeling of Transient and Steady-State Vapor Diffusion in Partially-Saturated Porous Media," ASME Proceedings of the 7th AIAA/ASME Thermophysics and Heat Transfer Conference, HTD-Vol. 357-2, pp. 221-231.

Webb, S.W., 1998, "Gas-Phase Diffusion in Porous Media: Comparison of Models," Proceedings of the TOUGH Workshop '98, LBNL-41995, pp. 269-274.

Webb, S.W., 1999, "Temperature Gradient Effects in Vapor Diffusion in Partially-Saturated Porous Media," submitted to the ASME/JSME Thermal Engineering Joint Conference, San Diego, Ca, March 15-19, 1999.

Webb, S.W. and Ho, C.K., 1998, "Review of Enhanced Vapor Diffusion in Porous Media," Proceedings of the TOUGH Workshop '98, LBNL-41995, pp. 257-262.

Webb, S.W. and Ho, C.K., 1998, "Pore-Scale Modeling Using TOUGH2," Proceedings of the TOUGH Workshop '98, LBNL-41995, pp. 288-293. 


\section{Distribution List}

Adeyinka E. Adenekan

Exxon Production Research Co.

P.O. Box 2189

Houston, TX 77252

Ronald W. Falta

College of Sciences

Clemson University

P.O. Box 341908

Clemson, SC 29634

Karsten Pruess

Earth Sciences Division

Lawrence Berkeley Laboratory

1 Cyclotron Road

Berkeley, CA 94720

G.S. Bodvarsson

Earth Sciences Division

Lawrence Berkeley Laboratory

1 Cyclotron Road

Berkeley, CA 94720

Mark D. White

Battelle, Pacific Northwest National Laboratory

P.O. Box 999 K9-36

Richland, WA 99352

Kent S. Udell

Department of Mechanical Engineering

University of California

Berkeley, CA 94720

O.A. Plumb

School of Mechanical and Materials

Engineering

Washington State University

Pullman, WA 99164-2920

Thomas A. Buscheck

Lawrence Livermore National Laboratory

P.O. Box 808, L-206

Livermore, CA 94550
John J. Nitao

Lawrence Livermore National Laboratory

P.O. Box 808, L-206

Livermore, CA 94550

George Zyvoloski

Los Alamos National Laboratory, EES-5

MS F665, P.O. Box 1663

Los Alamos, NM 87545

Ron Green

Southwest Research Institute

6220 Culebra Road

San Antonio, TX 78238-5166

Stephen Whitaker

Chemical Engineering Department

University of California

Davis, CA 95616

Marc Prat

I.M.F.T.

avenue du Professeur Camille Soula

31400 Toulouse, France

Professor John L. Wilson

Earth and Environmental Sciences

New Mexico Tech

Socorro, NM 87801

Jeff Roberts

Earth and Environmental Sciences

Lawrence Livermore National Laboratory

P.O. Box 808, L-202

Livermore, CA 94551

Dorthe Wildenschild

Earth and Environmental Sciences

Lawrence Livermore National Laboratory

P.O. Box 808, L-202

Livermore, CA 94551 


\section{Internal}

\begin{tabular}{lll}
$\underline{\text { MS }}$ & Org. & \\
0701 & 6100 & L.E. Shepherd \\
0735 & 6115 & P.B. Davies \\
0735 & 6115 & C.K. Ho (10) \\
0735 & 6115 & R.J. Glass, Jr. \\
0719 & 6131 & J.M. Phelan \\
1140 & 6500 & J.K. Rice \\
0977 & 6524 & W.R. Cook \\
0977 & 6524 & S.W. Webb (20) \\
0778 & 6851 & N.D. Francis \\
0778 & 6851 & M.T. Itamura \\
9402 & 8701 & C.M. Hartwig \\
0841 & 9100 & P.J. Hommert \\
0826 & 9111 & C.E. Hickox \\
0834 & 9112 & M.J. Martinez \\
0835 & 9113 & R.C. Dykhuizen \\
9018 & $8940-2$ & Central Technical \\
& & Files \\
0899 & 4916 & Technical Library (2) \\
0619 & 15102 & Review and Approval Desk \\
& \multicolumn{3}{c}{ for DOE/OSTI } \\
0188 & 4001 & LDRD Office
\end{tabular}

Distribution-2 